Check for updates

Cite this: Mater. Chem. Front. 2020, 4, 788

Received 23rd November 2019 Accepted 16th December 2019

DOI: 10.1039/c9qm00716d

rsc.li/frontiers-materials

\section{Recent advances in organic light-emitting diodes: toward smart lighting and displays}

\author{
Shi-Jie Zou, $\dagger^{a}$ Yang Shen,$\dagger^{a}$ Feng-Ming Xie, $\uparrow^{a}$ Jing-De Chen, ${ }^{a}$ Yan-Qing Li*ab and \\ Jian-Xin Tang (D) *ac
}

\begin{abstract}
Organic light-emitting diodes (OLEDs) have rapidly grown as one of the leading technologies for full-color display panels and eco-friendly lighting sources due to their outstanding features including superior color quality, wide viewing angle, mercury-free manufacture, fascinating flexibility, etc. A variety of materials, device architectures, as well as processing techniques have been investigated for optimizing device performance in order to fulfill the requirements of lighting and display applications. In this review, we first summarize the light emission mechanisms of electroluminescent materials. Then, the designed device architectures aiming at the realization of various light emission mechanisms are reviewed. An overview of recent advances in light extraction strategies is presented since all efficient OLEDs have a multi-thin-film structure, which leads to severe light trapping in devices. In addition, the progress of flexible OLEDs is reviewed from the aspect of flexible transparent electrodes because of their great potential in flexible displays. Most recent breakthroughs of solid-state lighting and displays are briefly addressed as well. A brief perspective on future research is also proposed for pursuing the commercialization of OLEDs.
\end{abstract}

\section{Introduction}

Organic light-emitting diodes (OLEDs) have attracted considerable interest owing to their amazing applications in full-color

${ }^{a}$ Jiangsu Key Laboratory for Carbon-Based Functional Materials \& Devices, Institute of Functional Nano \& Soft Materials (FUNSOM), Soochow University, Suzhou, 215123, Jiangsu, P. R. China. E-mail: yqli@suda.edu.cn, jxtang@suda.edu.cn

${ }^{b}$ School of Physics and Electronics Science, Ministry of Education Nanophotonics \& Advanced Instrument Engineering Research Center, East China Normal University, Shanghai, 200062, China

${ }^{c}$ Institute of Organic Optoelectronics (IOO), JITRI, Wujiang, Suzhou 215215, China $\dagger$ These authors contributed equally to this work. display panels and eco-friendly lighting sources since the pioneering work of Tang and Van Slyke in $1987 .{ }^{1}$ Great efforts in both academia and industry have been made towards the development of high-performance OLEDs in terms of luminance efficiency, color gamut, device stability, and fabrication techniques. $^{2-4}$ Recently, OLEDs have been commercialized as indoor lighting and displays in various consumer electronics, such as cell phones, digital cameras, and ultra-high-definition televisions. However, further optimization of OLED performance is still extremely challenging for competitive lighting sources and displays with desirable standards. ${ }^{5,6}$

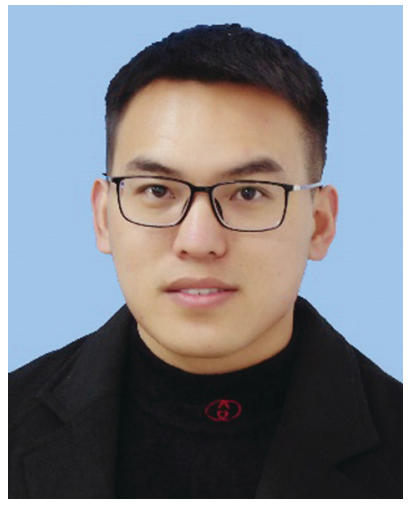

Shi-Jie Zou
Shi-Jie Zou received his BSc degree (2016) from the School of Science at Hebei University of Science and Technology, China. He is now a PhD candidate at the Institute of Functional Nano \& Soft Materials (FUNSOM) of Soochow University. His research interests focus mainly on the device physics of organic light-emitting diodes.

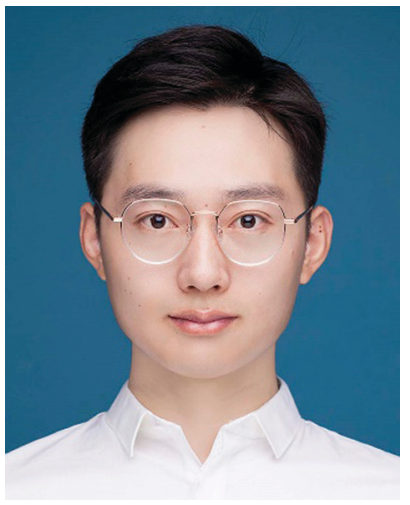

Yang Shen
Yang Shen received his BSc degree (2017) in Materials Science in Physics from Soochow University, China. He is now a master's degree student in Materials Science and Engineering at Soochow University, China. His research interests mainly include flexible transparent conductive electrodes, light manipulation for light-emitting diodes, and interfacial engineering for perovskite optoelectronic applications. 
One challenging factor for realizing high-performance OLEDs is the design and synthesis of advanced polymeric and small molecular emitting materials with appropriate electrical and optical properties to fully use the singlet and triplet excitons for energy conversion. ${ }^{7,8}$ Besides, the utilization of charges is also affected by the charge injection process, which is mainly determined by the electric properties of the transporting materials and the device architecture. ${ }^{9}$ At the same time, due to the concomitant influence of the device architecture on the operating voltage and luminance efficiency of OLEDs, the design of an appropriate architecture is another major challenge. However, despite the variant device architecture, all efficient OLEDs have a multi-thin-film structure, which results in the noteworthy drawback of OLEDs that a large fraction of generated photons are trapped within the device due to the refractive index mismatch between the flat functional films. ${ }^{10-12}$ As a result, only $20-30 \%$ of energy flow can radiate outside the device, which limits the development of highly efficient OLEDs. Therefore, the proposal of strategies for efficient extraction of emitted light is one of the main challenges for achieving highperformance devices. Furthermore, since OLEDs hold great potential in flexible displays and lighting, numerous efforts have been devoted to the research of flexible OLEDs, especially flexible transparent conductive electrodes (TCEs). ${ }^{13,14}$ Notably, the presented ITO-free electrodes play an important role in boosting the light extraction as well. In this regard, integrating light extraction strategies and flexible electrodes by using appropriate device architectures benefits the realization of highly efficient OLEDs, and thus fulfills the requirement of the real application scenarios.

In this review article, a brief summary of the emission mechanisms of organic electroluminescent materials and devices is first given for a better understanding of the background of device structure design. Subsequently, various device architectures and their characteristics are systematically reviewed along with a brief discussion of the fabrication technologies. We also overview the progress of light extraction strategies and simply classify them by two kinds of affecting mechanisms, the

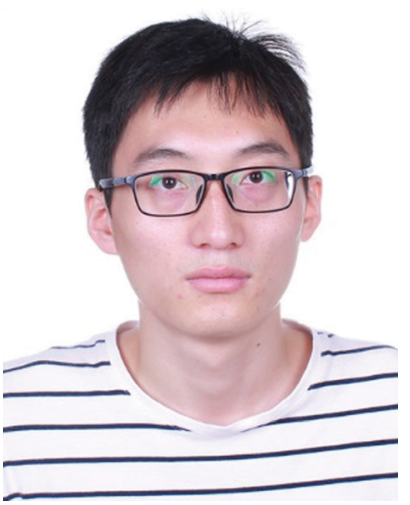

Feng-Ming Xie received his $M S c$ degree (2019) in Applied Chemistry from Suzhou University of Science and Technology, China. He is now a PhD student in Chemistry at Soochow University, China. He is mainly engaged in the design and synthesis of organic luminescence molecules and their applications in OLEDs.

Feng-Ming Xie

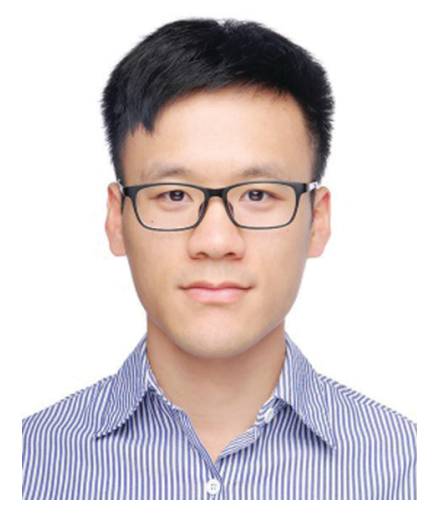

Jing-De Chen
Jing-De Chen received his $B S$ degree in physics from Zhejiang Normal University in 2012, and $P h D$ degree in Materials Science from Soochow University in 2018. Then, he joined the Institute of Functional Nano \& Soft Materials (FUNSOM) as a research assistant professor. His research interests focus mainly on energy conversion mechanisms in organic and perovskite solar cells and development of flexible transparent conductive substrates.

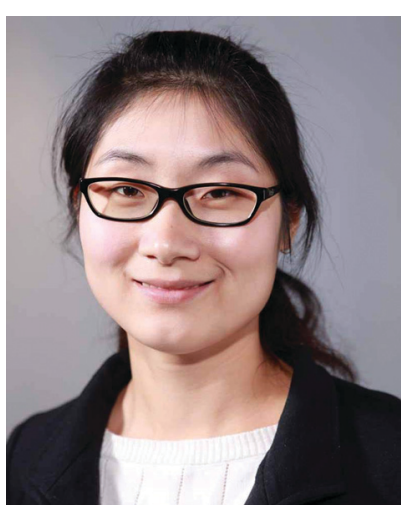

Yan-Qing Li
Yan-Qing Li received her BSc degree in physics from Zhejiang University, MPhil. degree and $P h D$ degree in Materials Science from City University of Hong Kong. She is now a professor at the Institute of Nano Functional \& Soft Materials (FUNSOM), Soochow University. Her main research interests lie in organic and inorganic/organic hybrid materials and devices with a focus on flexible electronics, involving the synthesis, characterization, and device integration of metal and semiconductor nanostructures and thin-films for a range of device applications including light-emitting diodes, solar cells, and smart windows.

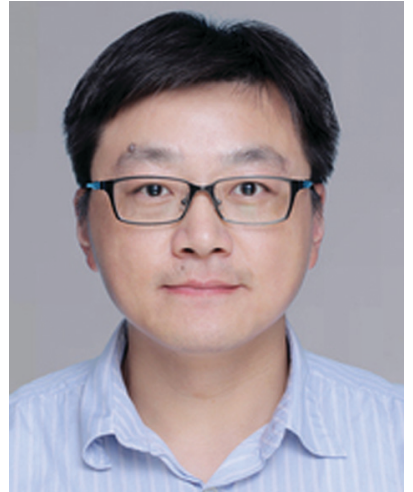

Jian-Xin Tang
Jian-Xin Tang received his BSc degree in physics from Zhejiang University, and PhD degree in Physics and Materials Science from City University of Hong Kong. In 2008, he was appointed a professor at the Institute of Nano Functional \& Soft Materials (FUNSOM), Soochow University. His research areas/interests span device physics and surface science of organic and perovskite lightemitting diodes for flat panel displays and solid-state lighting, and organic/perovskite photovoltaic cells for renewable energy, including localized electronic state and charge barrier formation at organic interfaces, and novel device architectures with interface modification for carrier transport and light manipulation. 
geometric optics and wave optics. Afterward, the development of flexible TCEs is also presented to illustrate the potential of OLEDs in flexible displays and lighting. Accordingly, the most recent breakthroughs of OLED technologies in the applications of displays and lighting are mentioned as well.

\section{Emission mechanisms of organic materials and devices}

\subsection{Singlet emission}

The emissions of conventional fluorescent emitters and up-conversion fluorescent emitters all originate from singlet excitons. The difference between these two emitters is that the up-conversion fluorescent emitters have a higher exciton utilization ratio than conventional fluorescent emitters. This is because the up-conversion fluorescent emitter possesses an up-conversion process from the triplet to the singlet state which harvests $75 \%$ of triplet excitons for luminescence. Thus developing an efficient up-conversion fluorescent emitter is a way to realize high efficiency and low-cost OLEDs. At present, there are three kinds of up-conversion emitters: triplet-triplet annihilation (TTA), hybrid local and charge transfer (HLCT), and thermally activated delayed fluorescence (TADF) (Fig. 1). Particularly, TADF emitters have attracted major attention in recent years due to their outstanding performance. The details of conventional fluorescent emitters and up-conversion fluorescent emitters (TTA, HLCT, and TADF) will be discussed below.

2.1.1. Conventional fluorescence. In organic semiconductors, the recombination of electrons and holes will generate singlet and triplet excitons with a ratio of $1: 3$ as charges are injected according to the spin statistical limit. ${ }^{15,16}$ The upper limit of the internal quantum efficiency (IQE, the ratio of the number of generated photons to the number of injected charges) of OLEDs using conventional fluorescent emitters is around $25 \%$ because of the radiative decay of singlet excitons and the non-radiative decay of triplet excitons (Fig. 1a). And the maximum external quantum efficiency (EQE) of such devices can only reach roughly $5 \%$, which can be described by the following equation:

$$
\mathrm{EQE}_{\text {max }}=\eta_{\mathrm{r}} \times \eta_{\mathrm{ST}} \times \Phi_{\mathrm{PL}} \times \eta_{\text {out }}=\mathrm{IQE}_{\text {max }} \times \eta_{\text {out }}
$$

where $\mathrm{EQE}_{\text {max }}$ and $\mathrm{IQE}_{\max }$ are the maximum external quantum efficiency and the maximum internal quantum efficiency, respectively. $\eta_{\mathrm{r}}$ is the proportion of electron-hole recombination, which is supposed to be unity in the ideal case. $\eta_{\mathrm{ST}}$ is the fraction of radiative excitons, $\Phi_{\mathrm{PL}}$ is the photoluminescence quantum yield (PLQY) of the emitting layer, and $\eta_{\text {out }}$ is the light out-coupling efficiency, which is around $20 \%$ for normal OLEDs with an ITO-based flat thin-film architecture. Regardless of the efficiency limitation, the fluorescent materials take advantage of long lifetimes, especially for stable blue emitters. ${ }^{17}$

2.1.2. Triplet-triplet annihilation. Triplet-triplet annihilation (TTA), also named P-type delayed fluorescence (DF), can fuse two triplet excitons into one singlet excited state, which makes the $\mathrm{IQE}_{\max }$ to $62.5 \% .{ }^{18}$ In the TTA process, a high-lying triplet state $\left(\mathrm{T}_{x}\right)$ and a ground state are generated by combining two low-lying triplet states $\left(\mathrm{T}_{1}\right)$, while $\mathrm{T}_{x}(x>1)$ has about twice the energy of $\mathrm{T}_{1}$. The generated high-lying triplet exciton then jumps to the singlet excited state through intersystem crossing and emits delayed fluorescence (Fig. 1b). The exciton lifetime of the TTA emitter contains prompt and delayed components, while the delayed part is much longer than the prompt part. The luminescence process of P-type DF is described by the following two-step scheme: ${ }^{19,20}$

$$
\begin{gathered}
\mathrm{T}_{1}+\mathrm{T}_{1} \rightarrow \mathrm{T}_{x}+\mathrm{S}_{0} \rightarrow \mathrm{S}_{n}+\mathrm{S}_{0} \rightarrow \mathrm{S}_{1}+\mathrm{S}_{0} \\
\mathrm{~S}_{1}+\mathrm{S}_{0} \rightarrow 2 \mathrm{~S}_{0}+h \nu
\end{gathered}
$$

where $\mathrm{S}_{0}, \mathrm{~S}_{n}$, and $\mathrm{S}_{1}$ represent the ground state, the high-lying singlet excited state and the first singlet excited state, respectively. And the photon energy is $h \nu$. Designing TTA molecules is a challenge because the energy gap between $2 \mathrm{~T}_{1}$ and $\mathrm{S}_{n}$ should (a)

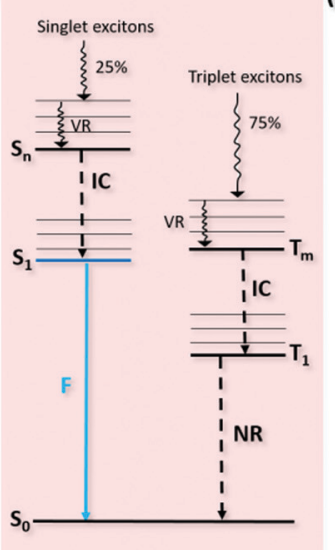

(b)

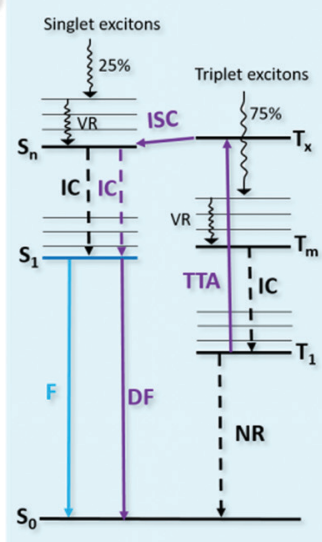

(c)

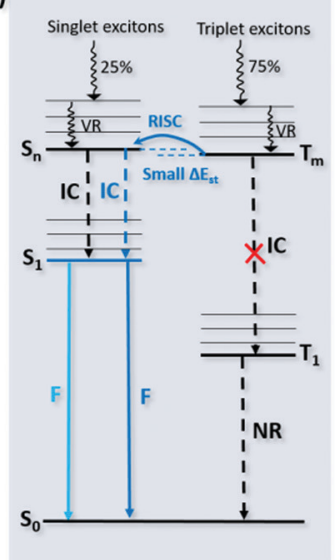

(d)

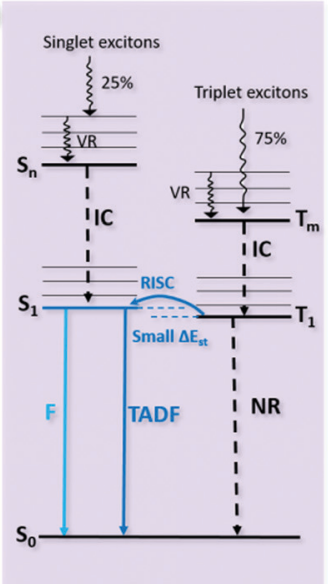

Fig. 1 Schematic diagrams of singlet emission mechanisms for (a) conventional fluorescence. (b) Triplet-triplet annihilation (TTA), (c) hybridized local and charge-transfer (HLCT) excited state, and (d) thermally activated delayed fluorescence (TADF). 


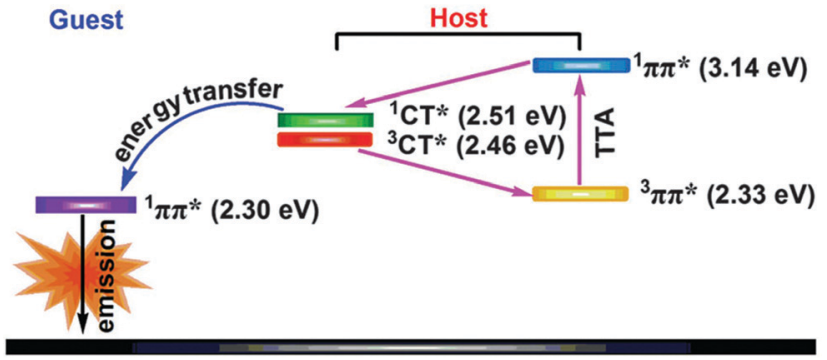

Fig. 2 The schematic energy transfer routes of the TTA process. Reproduced with permission from ref. 21. Copyright 2014 The Royal Society of Chemistry.

be small, as well as $2 \mathrm{~T}_{1}$ should be larger than $\mathrm{S}_{n}$. In the doping system, the TTA process may also originate from the host material molecules. ${ }^{21}$ To the end, the energy is transferred to the guest molecules by a Förster energy transfer process as the schematic process shown in Fig. 2.

The accumulation of triplet excitons is an essential condition for efficient TTA-OLEDs. However, the concentrated triplet excitons may result in serious efficiency roll-off. One recent work points out that the efficiency roll-off in TTA-OLEDs can be suppressed. $^{22}$ They reported a highly efficient blue-emitting material, phenanthroimidazole-anthracene (PIAnCN), and obtained non-doped system-based OLED devices with a low efficiency-roll-off. By combining rigid planar $\pi$-conjugated phenanthroimidazole with cyano substituted anthracene, the expectant molecule can utilize the non-emissive triplet excitons through a triplet fusion process. The non-doped blue device exhibits a high EQE of $9.44 \%$ at $1000 \mathrm{~cd} \mathrm{~m}^{-2}$ and the efficiency remains at $8.09 \%$ even the brightness reaches $10000 \mathrm{~cd} \mathrm{~m}^{-2}$. The results imply that the quenching of triplet excitons is suppressed in such a non-doped system. For device stability, it is also necessary to suppress the quenching of triplet excitons in the TTA process so as to prolong the lifetime of TTA-OLEDs. In 2016, Hsin and co-workers found that the addition of a $10 \%$ assistant host (volume concentration) into the EML could reduce the quenching of triplet excitons and block the diffusion of triplet pairs. ${ }^{23}$ Thus, the operation lifetime (T95) of blue TTA-OLEDs was increased by $89.3 \%$ from 28 to 53 hours at an initial luminance of $2000 \mathrm{~cd} \mathrm{~m}^{-2}$. In 2017, the same group developed a commercial blue TTA-OLED structure used for mass production. ${ }^{24}$ The standard structure had a T90 lifetime of 34 hours at $2000 \mathrm{~cd} \mathrm{~m}^{-2}$ and the T50 lifetime could reach 8000 hours at $1000 \mathrm{~cd} \mathrm{~m}^{-2}$. The commercial blue TTA-OLED structure exhibited an extended operation lifetime (T90) of 550 hours at an initial luminance of $2000 \mathrm{~cd} \mathrm{~m}^{-2}$ since a new ETL material, layer structure optimization and double EML structures were adopted in the structure.

In addition to the synthesis of materials with TTA properties, changing a fluorescent material to a TTA material is also a way to pursue efficient OLEDs. ${ }^{25,26}$ This strategy requires exciplexes or sensitizers to assist the accumulation of triplet excitons in the luminescent layer.

2.1.3. Hybridized local and charge-transfer. Hybridized local and charge-transfer (HLCT) is another strategy to harvest the $75 \%$ of non-emissive triplet excitons for luminescence. ${ }^{27-33}$
As shown in Fig. 1c, for the HLCT state, the hot excitons injected to the high-lying triplet state $\mathrm{T}_{m}(m \geq 2)$ are transferred to the singlet state through reverse intersystem crossing (RISC). Excitons located in the second or higher excited state are called hot excitons, and the process of exciton transfer from the high-lying triplet state to singlet state is called the hot exciton process. ${ }^{27} \mathrm{Ma}$ and co-workers proposed that the realization of the hot exciton process requires two preconditions: a large energy gap between $\mathrm{T}_{m}$ and $\mathrm{T}_{1}$, and a small energy gap between $\mathrm{T}_{m}$ and $\mathrm{S}_{n}$ (where $n \geq 1$ ). ${ }^{28}$ Although Kasha's rule points out that the majority of excitons tend to preferentially occupy the lowest excited states rather than the high-lying excited states, ${ }^{34}$ the large energy gap between $\mathrm{T}_{m}$ and $\mathrm{T}_{1}$ can impede the internal conversion (IC) process. Moreover, the small energy gap between $\mathrm{T}_{m}$ and $\mathrm{S}_{n}$ is helpful for the processing of RISC. As a result, the triplet excitons located in the $\mathrm{T}_{m}$ state will not transfer to the lowest triplet state, but to the singlet state.

Due to the exact demands on energy level, the design and the synthesis of an HLCT molecule are very challenging. HLCT molecules should ideally contain intercrossed local excited (LE) states (for efficient fluorescence emission) and charge transfer (CT) states (for efficient RISC), which means after the molecule is excited, orbital overlap and orbital spatial separation are satisfied at the same time. A molecule with a twisting donor unit and acceptor unit may possess such HLCT excited states. Notably, the appropriate twisting angle and the appropriate ratio of these two excited states are crucial. ${ }^{29}$ The non-linear relationship between the solvent polarity and Stokes shift, along with the single-exponential curve of PL lifetime, indicates that this type of molecule possesses HLCT states but not a mixture of LE and CT states. ${ }^{28,29}$ Different from TTA and TADF materials, neither delayed PL nor delayed EL lifetime was measured in HLCT materials. The short exciton lifetime is attributed to the faster rate of RISC ( $\left.k_{\text {RISC }}\right)$ compared to the radiative rate, which is caused by the high-lying triplet energy transfer. Meanwhile the fast RISC process from the high-lying triplet to singlet state can suppress triplet-triplet and singlettriplet quenching caused by the accumulated triplet excitons in the device, which further reduces efficiency roll-off and increases device stability. ${ }^{27}$ But there is a lack of direct experimental evidence for the proposed hot exciton RISC process. In addition, although the HLCT emitters have shown a high $\eta_{\mathrm{ST}}$ of 93\%, the overall EQE is still limited by the low PLQY. ${ }^{28}$

In addition, Tang and co-workers reported several aggregationinduced emission-assisted hybrid local and charge transfer (AIE-HLCT) molecules, which consist of an AIE moiety and an HLCT-type core. The corresponding non-doped OLED devices exhibited a small efficiency roll-off and a maximum EQE of $7.16 \%{ }^{33}$

2.1.4. Thermally activated delayed fluorescence. Thermally activated delayed fluorescence (TADF) or E-type delayed fluorescence is regarded as a promising fluorescent emitter due to the efficient endothermic up-conversion, which can utilize all triplet excitons for emission. ${ }^{35-40}$ As shown in Fig. 1d, the excitons located on the first triplet excited state can transfer to the first singlet state in TADF molecules through up-conversion. To activate the up-conversion of the RISC process at room 
temperature, $\Delta E_{\mathrm{ST}}$ smaller than $0.2 \mathrm{eV}$ is required, which means a small molecule's orbital overlap between the highest occupied molecular orbital (HOMO) and the lowest unoccupied molecular orbital (LUMO). ${ }^{35-37}$ The constant of RISC ( $\left.K_{\text {RISC }}\right)$ can be expressed using the Boltzmann distribution relation:

$$
k_{\mathrm{RISC}} \propto \exp \left(-\frac{E_{\mathrm{ST}}}{k_{\mathrm{B}} T}\right)
$$

where $k_{\mathrm{B}}$ is the Boltzmann constant and $T$ is the temperature in centigrade. It is obvious that a small $\Delta E_{\mathrm{ST}}$ is required for fast RISC.

The first pure organic TADF molecule (PIC-TRZ) was reported by Adachi and co-workers in $2011 .^{41}$ They found that the twisted donor-acceptor structure of PIC-TRZ can lead to a reduced orbital overlap between the HOMO and LUMO. The calculated $\Delta E_{\mathrm{ST}}$ is as low as $0.11 \mathrm{eV}$. A high EQE of $5.3 \%$ was obtained by using PIC-TRZ as an emitter while the theoretical EQE was only $2 \%$ for a conventional fluorescent emitter with a PLQY of $39 \%$. They attributed the results to the efficient up-conversion of the RISC process. Afterward, a series of pure organic TADF emitters with both small $\Delta E_{\mathrm{ST}}$ and high PLQY were reported by Adachi and co-workers. ${ }^{35}$ An OLED with the green TADF emitter (4CzIPN) exhibited an outstanding EQE of 19.3\%, and the values were $11.2 \%$ and $8.0 \%$ for orange and sky-blue OLEDs, respectively. These results indicate that TADF is a feasible strategy for achieving high-efficiency OLEDs. Up to now, the highest EQE values of $\sim 38 \%$ for blue, $\sim 37 \%$ for green, and $\sim 28 \%$ for red OLEDs have been achieved by using various TADF-based emitters, which are far beyond the device efficiency of conventional fluorescent OLEDs and even comparable with phosphorescent OLEDs. ${ }^{38-40}$

However, the stability of TADF-OLEDs cannot meet the requirements of mass production, especially blue TADF devices. We summarize several blue TADF devices based on the stability reported in recent years. In 2015, the operation lifetime (T80) of blue TADF devices is only 52 hours at an initial luminance of $500 \mathrm{~cd} \mathrm{~m}^{-2}$ reported by Lee and co-workers. ${ }^{42}$ In 2016, a T50 of 770 hours at an initial luminance of $500 \mathrm{~cd} \mathrm{~m}^{-2}$ was reported by adopting a sterically shielded sky-blue TADF emitter. ${ }^{43}$ In 2017 , Adachi and co-workers reported a stable blue TADF-OLED with an operation lifetime of 454 hours at an initial luminance of $1000 \mathrm{~cd} \mathrm{~m}^{-2}$ by employing an n-type material as the host. ${ }^{44}$ In 2018, Adachi and co-workers synthesized a stable sky-blue TADF emitter, namely $3 \mathrm{Ph}_{2} \mathrm{CzCzBN} .{ }^{45}$ To the best of our knowledge, the device showed the longest T80 of 118 hours at an initial luminance of $1000 \mathrm{~cd} \mathrm{~m}^{-2}$. Therefore, the poor stability of blue TADF-OLEDs is still a problem to be solved.

\subsection{Doublet emission}

Radical-based emitters, whose emission originates from doublet excited states, have attracted more attention and obtained efficiency breakthrough in recent years. ${ }^{46,47}$ Unlike fluorescence and phosphorescence emitting molecules, radical-based emitter molecules have odd electrons and are in open-shell states due to unpaired outer orbital electrons. Fig. 3 is the spin configurations of closed-shell and open-shell molecules. ${ }^{46}$ When a closed-shell (a)

LUMO

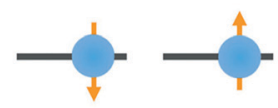

HOMO

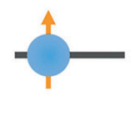

Singlet

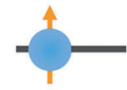

Triplet (b)

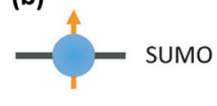

Doublet
SOMO

Fig. 3 The spin configurations of (a) closed-shell molecules and (b) openshell molecules. Reproduced with permission from ref. 46. Copyright 2015 Wiley-VCH Verlag GmbH \& Co. KGaA, Weinheim.

molecule (fluorescent or phosphorescent molecule) is excited, one electron will be transferred to the LUMO, and the other will remain at the HOMO. In this case, the spin quantum number obtained from these two electrons can be 0 or 1 , corresponding to the singlet and triplet states respectively (Fig. 3a). ${ }^{48}$ However, the triplet excitons of pure organic fluorescent molecules can't transit to the ground state for emission as a result of the Pauli exclusion principle. ${ }^{15}$ For an open-shell molecule, the excited state is called a doublet because the spin quantum number is $1 / 2$. And when the only ground-state electron at the singly occupied molecular orbital (SOMO) is excited to the lowest singly unoccupied molecular orbital (SUMO), it can jump back to the SOMO without any forbidden rules (Fig. 3b). Thus the IQE of the radical emitter based on an open-shell structure is theoretically $100 \%$.

Although stable emission radicals have been reported since $2006,{ }^{49-51}$ the first radical OLED was fabricated in $2015 .{ }^{46}$ Nonetheless, radical OLEDs got a big breakthrough in $2018 .{ }^{47}$ $\mathrm{Ai}$ and co-workers fabricated OLEDs using a stable radical molecule (TTM-3NCz) and obtained almost 100\% IQE, as well as an excellent EQE of $27 \%$ in the deep red/near-infrared spectral range. By combining the radical TTM moiety with the donor group of $3 \mathrm{NCz}$, the radical molecule can selectively control the injection of holes and electrons to the HOMO and SOMO, respectively. Doublet excitons with a small band gap are formed based on the HOMO and SOMO, therefore, the emission color is in the deep red/near-infrared spectral range. However, no stable blue light-emitting radical has been reported up to now, which limits the further development of radical OLEDs.

\subsection{Triplet emission}

2.3.1. Phosphorescent emitters. Electroluminescence from the triplet excited states of phosphorescent emitters based on Os(II) and Pt(II) complexes was observed by Ma and Forrest in $1998 .^{52,53}$ The introduction of heavy metal atoms to organic skeletons can not only break through the forbidden transition from $T_{1}$ to $S_{0}$, but also promote the ISC process from $S_{1}$ to $T_{1}$ because of the strong spin-orbit coupling effect. ${ }^{54}$ So the IQE of phosphorescent OLEDs can reach $100 \%$ with the full utilization of excitons. In the following decades, the rapid development of phosphorescent emitters has caused a revolution in the display and lighting industry. So far, numerous phosphorescent transition metal complexes have been used for constructing highly 
efficient OLEDs, such as $\operatorname{Ir}(\mathrm{III}),{ }^{55} \mathrm{Pt}(\mathrm{II}),{ }^{56} \mathrm{Au}(\mathrm{III}),{ }^{57} \mathrm{Os}(\mathrm{II}),{ }^{58}$ $\operatorname{Re}(\mathrm{I}),{ }^{59,60} \mathrm{Ru}(\mathrm{II}),{ }^{61}$ and $\mathrm{Cu}(\mathrm{I})^{62,63}$ complexes. Among them, $\operatorname{Ir}\left(\right.$ III) complexes, e.g. $\operatorname{Ir}(\text { ppy })_{3}$, FIrpic and $\operatorname{Ir}(\text { piq) })_{3}$, are the most widely used because of their high emission efficiency, relatively short triplet exciton lifetime, and wide color range. ${ }^{64}$ However, these low abundant rare metals are costly, unsustainable and unfriendly to the environment; thus, some abundant and cheap alternative metals are needed. Copper has attracted the attention of many material scientists due to its low-cost and hypotoxicity. But $\mathrm{Cu}(\mathrm{I})$ complexes are originally considered as a poor candidate for OLEDs because of the weak spin-orbit coupling of the light atoms. The slower ISC rate (from $\mathrm{S}_{1}$ to $\mathrm{T}_{1}$ ) and radiative rate (from $\mathrm{T}_{1}$ to $\mathrm{S}_{0}$ ) of $\mathrm{Cu}$-based emitters as compared to Ir-based and Pt-based emitters indicates the poor phosphorescence efficiency of Cu-based emitters. ${ }^{65}$ Therefore, some studies focus on another emission channel of $\mathrm{Cu}(\mathrm{I})$ complexes, the E-type delayed fluorescence. ${ }^{65-67}$ As in the case of conventional TADF emitters, the small $\Delta E_{\mathrm{ST}}$ and high PLQY of $\mathrm{Cu}(\mathrm{I})$ complexes are crucial to realize an efficient delayed fluorescence. ${ }^{67}$ By introducing the TADF mechanism, $\mathrm{Cu}$-based green and orange OLEDs exhibited a maximum EQE of over $20 \%,{ }^{66,68}$ and the value was improved to $9 \%$ for blue OLEDs. ${ }^{65}$

In terms of device operation stability, the reported T95 (operational duration of the device till the luminance is decayed to $95 \%$ of the initial luminance, which is $1000 \mathrm{~cd} \mathrm{~m}^{-2}$ ) of green and red PhOLEDs is longer than 10000 hours. ${ }^{69}$ Nevertheless, long-lived blue PhOLEDs have not been realized since their first demonstration in $2001 .^{70}$ To the best of our knowledge, the champion T80 of sky-blue PhOLEDs with a single emitting layer is only about $334 \mathrm{~h}$ with CIE coordinates of $(0.16,0.30) .^{71}$ High energy excitons and polarons generated by exciton-exciton annihilation and exciton-polaron annihilation are considered as the main degradation mechanisms for PhOLED devices. ${ }^{72-74}$ The degradation probability of blue PhOLEDs is higher than that of red and green PhOLEDs since blue-emitting layers generate more high energy excitons and polarons through annihilation reactions, which will break the weak chemical bonds between the Ir core metal and organic ligands. ${ }^{75}$ Low abundance of precious metals and poor stability of blue devices are pushing scientists to research new solutions or provide alternatives to phosphorescent emitters.
2.3.2. Long-lasting phosphorescence. Compared to the heavy metal-based room temperature phosphorescent emitters, purely organic room temperature phosphorescent emitters show a constitutionally longer phosphorescence lifetime, which is of the order of seconds. ${ }^{76-79}$ Usually, when pure organic lightemitting materials are exposed to ambient temperature, the triplet excitons will be quenched and lead to nonradiative decay processes. Fortunately, the nonradiative decay processes of guest materials can be suppressed by employing rigid matrix materials. $^{76,80-83}$ A semiconducting rigid matrix material (CzSte) consisting of a carbazole unit and a hydrophobic steroid group was reported by Adachi and co-workers in 2015, and the corresponding Long-lasting Phosphorescence (LLP) OLED was realized. ${ }^{76}$ The LLP-OLED exhibited a dual emission from both blue fluorescence and green phosphorescence. The calculated ratio of phosphorescence to the total emission is about $5 \%$. In addition, the green phosphorescence can continue to glow for a little while after the power supply is cut off because of its long lifetime. Therefore, LLP-OLEDs are promising for indoor lighting, especially for bedroom lighting.

\section{Design of device structures}

OLEDs typically have a multilayered device structure as shown in Fig. 4a, including an anode, hole injection layer (HIL), hole transport layer (HTL), light-emitting layer (LEL), electron transport layer (ETL), electron injection layer (EIL) and cathode. Electrons and holes are injected into the LEL from the two electrodes and then recombined on emitter molecules to emit light, while the emission color is determined by the emitter molecule. Obviously, the design and optimization of the device architecture are one of the key ways to obtaining highly efficient and stable OLEDs; thus, numerous strategies have been presented to boost the charge transport, promote the charge injection, as well as facilitate the radiative recombination.

\subsection{Doping}

To boost the efficiency output of OLEDs, strategies aiming at reducing the operation voltage have been proposed for enhancing the carrier conductivities of organic layers and matching the energy levels of functional layers. By introducing such a
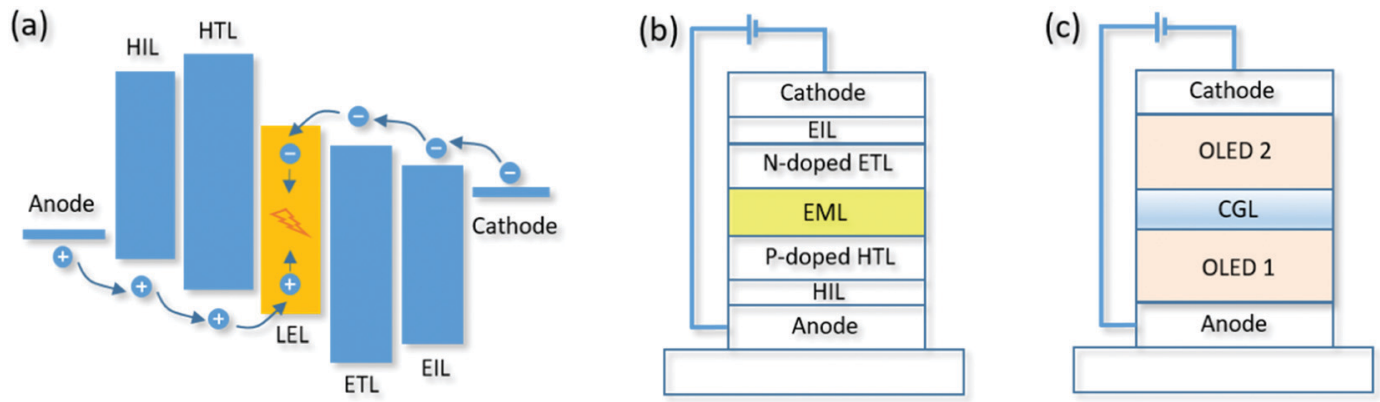

Fig. 4 Representative device architectures of OLEDs. (a) The working mechanism of OLEDs, (b) charge injection and transport in OLEDs enhanced by $\mathrm{p}$ - and $\mathrm{n}$-type electrical doping, and (c) tandem OLED structures with stacked emission units connected by a charge generation layer (CGL). 
strategy, the energy loss during charge injecting and transporting processes is dramatically reduced. Molecular doping of organic layers is an effective strategy to overcome the intrinsic limitations of transport conductivity of organic layers and address the large carrier injection barriers at the organic/electrode interfaces. ${ }^{84,85}$ As the schematic architecture is shown in Fig. $4 \mathrm{~b}$, the device with a ptype doped HTL, an intrinsic emission layer, and an n-type doped ETL is generally regarded as an efficient OLED, which has highly reduced operating voltage at high luminance. ${ }^{86}$ To date, the representative p-type and n-type dopants used for electrical doping are transition metal oxides (TMOs) (e.g., $\mathrm{MoO}_{3}, \mathrm{WO}_{3}$, $\mathrm{V}_{2} \mathrm{O}_{5}, \mathrm{ReO}_{3}$, etc. $)$ and alkali metal compounds (e.g. $, \mathrm{Cs}_{2} \mathrm{CO}_{3}, \mathrm{LiF}$, CsF, etc.), respectively. ${ }^{87-95}$

3.1.1. p-Type doping. TMOs have unique advantages for p-type doping of organic HTLs in OLEDs because of their high ionization energy $(>6 \mathrm{eV})$ and wide band gap. ${ }^{87} \mathrm{MoO}_{3}$ and $\mathrm{WO}_{3}$ are the two most popular choices to serve as buffer layers and p-type dopants in organic devices. For example, with the introduction of $\mathrm{MoO}_{3}$ doping, the electrical conductivity of a $4,4^{\prime}$-bis( $N$-carbazolyl)-1,1'-biphenyl (CBP)-based hole-only device can be improved to five orders of magnitude larger as compared to the intrinsic materials (Fig. 5a). ${ }^{88}$ It has been reported that the remarkable increase in film conductivity of p-type doped organic semiconductors originates from the formation of a charge-transfer complex as evidenced by the existence of a broad absorption peak in the near-infrared region of doped organic films. ${ }^{89}$ The doping process occurs with electron transfer from the HOMO of the host molecules to the low-lying unoccupied states of TMO dopants. Ultraviolet photoelectron spectroscopy (UPS) measurements provide direct evidence of identifying the electronic structures of p-type doped organic layers with various doping concentrations. A summary of the UPS measurements on electronic structures of the $\mathrm{MoO}_{3}$ doped CBP layer (Fig. 5a, middle) indicates that the Fermi level $\left(E_{\mathrm{F}}\right)$ is shifted towards the HOMO level rapidly with increasing dopant concentration. The result on the right hand side of Fig. 5a reveals that the electrical conductivity is simultaneously increased. In addition, $\mathrm{ReO}_{3}$ has been used for p-type surface doping, resulting in a nearly unit hole injection efficiency along with a perfect Ohmic contact at the interface of ITO and $N, N 9$-di(naphthalene-1-yl)-N,N9-diphenyl-benzidine (NPB). ${ }^{90}$ The formation of the ohmic contact is attributed to the high carrier density near the $\mathrm{ReO}_{3} / \mathrm{NPB}$ interface with a hole injection barrier of less than $0.4 \mathrm{eV}$.

3.1.2. n-Type doping. Compared with using conventional reactive metals as n-type dopants, the incorporation of alkali metal compounds, e.g., $\mathrm{Cs}_{2} \mathrm{CO}_{3}, \mathrm{CsF}, \mathrm{CsN}_{3}$, is a more effective way to realize n-type doping of organic ETLs because of the merits of easy material processing and high operational stability. ${ }^{91,92}$ The alkali metal compounds that act as n-type dopants in OLEDs can efficiently facilitate electron injection by reducing electron injecting barriers. Moreover, the bulk conductivity of organic layers is increased due to the reduction in ohmic losses. As the result shown in Fig. 5b, it is clear that the current densityvoltage $(J-V)$ characteristics of OLEDs with an ETL of CsFdoped tris-(8-hydroxyquinoline) aluminum $\left(\mathrm{Alq}_{3}\right)$ show doping
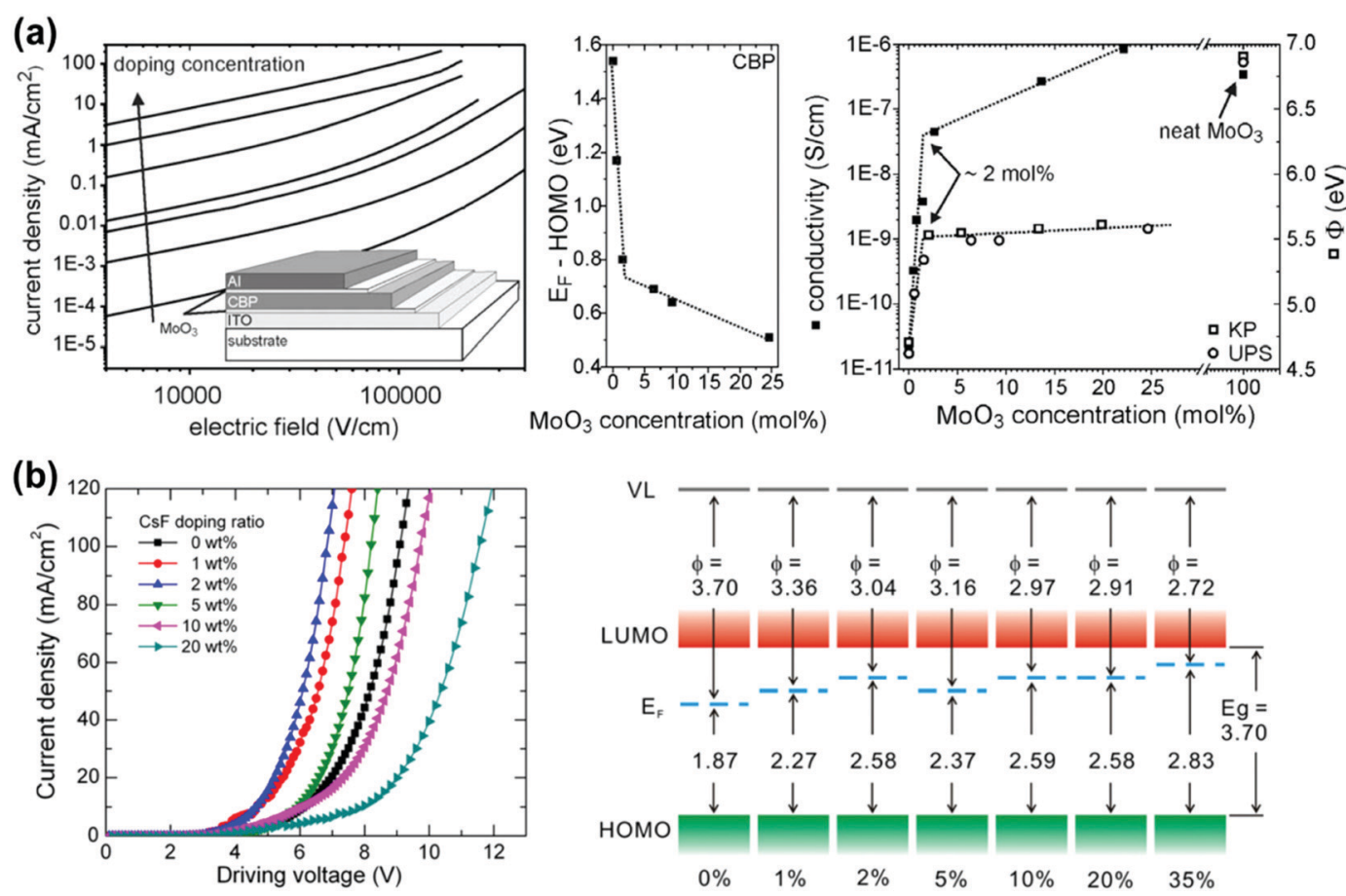

Fig. 5 (a) Electrical characteristics of hole-only CBP devices (left), the energy difference between $E_{\mathrm{F}}$ and HOMO edge (middle), and conductivity (right) as a function of $\mathrm{MoO}_{3}$ concentration. Reproduced with permission. Reproduced with permission from ref. 88. Copyright 2009 Elsevier. (b) Electrical characteristics of the OLED with a CsF-doped $\mathrm{Alq}_{3}$ ETL (left) and energy difference between $E_{\mathrm{F}}$ and the HOMO edge (right) as a function of CsF concentration. Reproduced with permission. Reproduced with permission from ref. 93. Copyright 2013 Elsevier. 
concentration-dependent current density. ${ }^{93}$ The devices with appropriately doped CsF:Alq ${ }_{3}$ ETLs show better performance as compared to the reference device with an undoped $\mathrm{Alq}_{3}$ layer. Similar to the $\mathrm{MoO}_{3}$ doping in HTLs (Fig. 5a, middle), two different regimes can be identified with the CsF doping in $\mathrm{Alq}_{3}$ layers (Fig. 5b, right). The HOMO edge of the $\mathrm{Alq}_{3}$ layer keeps away from $E_{\mathrm{F}}$ at a low CsF doping concentration ( $<2 \mathrm{wt} \%$ ), which corresponds to the relatively large increase in the conductivity. As for the $\mathrm{Alq}_{3}$ with a high concentration ( $>2 \mathrm{wt} \%$ ) of CsF doping, a small $E_{\mathrm{F}}$ shift was found, along with a shift in the HOMO edge from $1.9 \mathrm{eV}$ to $2.8 \mathrm{eV}$ below $E_{\mathrm{F}}$. Besides, the work function $(\phi)$ of the doped films was changed from $3.7 \mathrm{eV}$ to $2.7 \mathrm{eV}$. As a result, the electron injection barrier at the $\mathrm{Alq}_{3} \mathrm{ETL}$ is reduced, and the negative carrier density is also increased. Similar doping behaviors have been observed in n-type doping of organic layers with a variety of alkali metal compounds. ${ }^{87-97}$ Clarifying the working mechanisms of alkali metal compounds as n-type dopants in organic layers is advantageous for further design and optimization of novel electrical doping. According to the measurement results of photoelectron spectroscopy of n-type doping, the exact working mechanism is changed depending on the alkali metal compounds. ${ }^{91-95}$ In particular, the incorporation of alkali metal compounds like $\mathrm{Cs}_{2} \mathrm{CO}_{3}$, CsF or $\mathrm{CsN}_{3}$ by using the thermal evaporation method will lead to their decomposition. $\mathrm{Cs}_{2} \mathrm{CO}_{3}$ will be partially decomposed with the product, cesium oxide, while $\mathrm{CsN}_{3}$ and $\mathrm{CsF}$ generate cesium atoms which interact strongly with organic molecules. ${ }^{93-96}$

Apart from the difference in electrical characteristics, the operational stability of organic devices with an n-type doped ETL is strongly affected by the doping mechanism of different alkali metal compounds. ${ }^{91}$ Without generating metallic Cs during the $\mathrm{Cs}_{2} \mathrm{CO}_{3}$ doping process, the diffusion of metal atoms through the ETLs into the adjacent emission layers can be suppressed, avoiding the consequential non-radiative recombination of excitons. As a comparison, the thermally evaporated $\mathrm{CsN}_{3}$ will be completely decomposed and the Cs atoms are simultaneously released in the organic ETLs, which result in the formation of non-radiative recombination centers and the quenching of the excitons, as well as degraded device performance. ${ }^{98}$ Therefore, the OLEDs using $\mathrm{Cs}_{2} \mathrm{CO}_{3}$ as the n-type dopant show advantages in both electrical properties and operational stability.

\subsection{Tandem and multi-junction structures}

OLEDs using tandem structures offer a highly attractive option for future general lighting due to the extremely high brightness and efficiency at a low driving current, along with the long operational lifetime. ${ }^{99,100}$ In a tandem OLED, vertically stacked emission units are electrically connected in series by a charge generation layer (CGL), and the brightness and current efficiency at a given current density can linearly scale with the number of emission units (Fig. 4c). Upon the application of an electric field, electrons and holes generated by the CGL would be injected into the neighboring ETL and HTL of the respective emission unit and subsequently recombine with holes injected from the anode side or electrons injected from the cathode side for light emission. Thus, the design of an efficient CGL is imperative to the high-performance electron-to-photon conversion process in tandem OLEDs.

The requirements of an effective CGL mainly include high charge generation capacity, low optical absorption, low series resistance, and excellent operational stability. ${ }^{101}$ Typically, the CGLs in tandem OLEDs comprise bilayer structures, including metal/metal oxide, organic/metal oxide and organic/organic types. ${ }^{100-104}$ Following the discussion in Section 3.1., this section would focus on a representative CGL structure with an organic/ metal oxide bilayer. In this CGL, TMO materials are often used adjacent to the HTL of the neighboring emission unit, while the organic layers commonly doped by n-type alkali metals or metal compounds are incorporated adjacent to the ETL of the neighboring emission unit. The functionality of $\mathrm{MoO}_{3}$-based CGL, for instance, is fully clarified, showing that the vacuumdeposited $\mathrm{MoO}_{3}$ layer is responsible for charge generation due to spontaneous electron transfer from various defect states to the conduction band via thermal diffusion. ${ }^{95}$ The external electric field induces charge separation through extraction of charge carriers into the neighboring charge transport layers. The n-doped organic layer can not only facilitate electron extraction into the neighboring ETL but also block the leakage of holes across the CGL into the HTL of the adjacent emission unit. From the perspective of long lifetime merit of tandem OLEDs, the influence of constituent materials and the operational stability of CGLs has also been illustrated. Taking different n-dopants including $\mathrm{Mg}, \mathrm{Cs}_{2} \mathrm{CO}_{3}$, and $\mathrm{CsN}_{3}$ as an example, the stability of tandem devices is highly sensitive to the doping constituent materials used in the CGLs, in spite of the similar energy level barrier. ${ }^{105}$ The findings suggest that the operational stability of the n-doped layers in CGLs is the main factor responsible for device degradation due to the different doping mechanisms.

In addition to the mechanistic study, one of the most intriguing advantages of tandem OLEDs is the extremely high luminous efficiency. By employing efficient phosphorescent emitters, a triple-junction green OLED using HATCN-based CGLs exhibits a current efficiency of $256 \mathrm{~cd} \mathrm{~A}^{-1}$ at a luminance of $100 \mathrm{~cd} \mathrm{~m}^{-2} \cdot{ }^{106}$ To unlock the full potential of tandem OLEDs, a phosphorescent OLED with a combination of triple emission units and a light outcoupling structure has achieved an ultrahigh current efficiency of $366 \mathrm{~cd} \mathrm{~A}^{-1}$ at $1000 \mathrm{~cd} \mathrm{~m}^{-2}$ together with a maximum EQE of $119.7 \% .^{107}$ Based on this strategy, microcavity-induced angle-dependent emission in tandem OLEDs can be alleviated, and the operational lifetime can be simultaneously enhanced by a factor of $>10$ when comparing the double junction device with the conventional device with a planar single-unit structure. ${ }^{108}$

\subsection{Exciplex}

Exciplexes are generated in a mixed film or an interface consisting of both a p-type donor material and an n-type acceptor material. Generally, the formation of an exciplex requires a large offset between the HOMO of the donor material and the LUMO of the acceptor material. Notably, the emission energy of the exciplex is smaller than that of each component. In recent years, 
exciplexes have been frequently investigated in OLEDs, since exciplexes can act as both an emitter and a host for fluorescent, TADF and phosphorescent emitters. ${ }^{40,109-119}$ Moreover, exciplexbased OLEDs exhibit prominent advantages, such as good carrier balance, low driving voltage, and high efficiency.

3.3.1. Exciplex emitters. An exciplex system can be regarded as a 'new fluorescent material'. It may possess up-conversion properties if the exciton lifetime has delayed components and $\Delta E_{\mathrm{ST}}$ meets the necessary conditions. Therefore, a high EQE of over $5 \%$ can also be achieved by using an exciplex as a direct emitter. ${ }^{109-112}$ As reported by Adachi and co-workers, the OLEDs using a m-MTDATA:PPT exciplex as the emitter exhibit a high EQE of $10.0 \%$ because of the efficient exciton up-conversion. ${ }^{109}$ In their work, the small energy gap $\Delta E_{\mathrm{ST}}(<0.1 \mathrm{eV})$ and the delayed fluorescent lifetime of the exciplex demonstrate the existence of the TADF process. In 2015, Zhang and co-workers reported an up-conversion exciplex consisting of TPAPB and TPBi. This exciplex emitter exhibited an EQE of $7.0 \%$, a high exciton contribution of over $25 \%$ and a large $\Delta E_{\mathrm{ST}}$. They pointed out that the up-conversion mainly originated from TTA. ${ }^{110}$ Zhang and co-workers reported OLEDs based on another exciplex emitter, which shows an excellent EQE of 15.4\%, and attributed the high efficiency to the efficient up-conversion via RISC. ${ }^{111}$ In addition, Wong and co-workers reported an exciplex formed at the interface which leads to a device with an orange emission and an EQE of $7.7 \% .^{112}$ The strategy of using exciplexes as emitters provides a way for breaking the conventional fluorescent limit of $25 \%$ exciton utilization.

3.3.2. Exciplex hosts. On the other hand, exciplexes have also been widely used as the host for fluorescent, TADF, and phosphorescent emitters because of their unique advantages. ${ }^{114-119}$ By employing exciplex systems containing n-type and p-type materials as the host for OLEDs, the driving voltage is reduced because of the boosted carrier injection. Moreover, the carrier balance in the light-emitting layer can be well achieved by adjusting the ratio of the n-type material and p-type material, which further improves the emission efficiency and enhances the operation stability of the device. More importantly, the exciplex host can change singlet/triplet exciton proportions in itself as well as in the emitter due to the efficient up-conversion process. In recent years, high-performance TADF and phosphorescent
OLED devices based on exciplex hosts have been widely reported. ${ }^{115-119}$ For example, a green TADF-based OLED exhibited an excellent EQE of $30 \%$ by employing an mCP: B3PYMPM exciplex system as the host. ${ }^{115}$ A solution-processed TADF-OLED with reduced efficiency roll-off was achieved based on a TAPC/TmPyPB interfacial exciplex host. ${ }^{116}$ In addition, Liao and co-workers reported a deep-red TADF-OLED with a record EQE of $28.1 \%$ by adopting an exciplex host strategy. ${ }^{40}$ A white Ph-OLED with a power efficiency of $105 \mathrm{~lm} \mathrm{~W}^{-1}$ without a light extraction structure was reported by Fung and co-workers in 2017. ${ }^{117}$ Furthermore, a highly efficient white Ph-OLED with ultra-high color stability was demonstrated by adopting an exciplex as the host. ${ }^{118}$

Particularly for conventional fluorescent emitters, the exciton utilization can reach nearly $100 \%$ when exciplex hosts have TADF characteristics. ${ }^{114}$ As shown in Fig. 6a, two conditions should be satisfied for achieving high-efficiency fluorescent OLEDs. On the one hand, an efficient Förster energy transfer (FRET) process from $S_{1}$ of the exciplex host to $S_{1}$ of the fluorescent emitter is crucial, which requires an efficient RISC process of the exciplex host and a larger spectrum overlap between the absorption of the fluorescent dopant and the emission of the exciplex host. ${ }^{114,120}$ On the other hand, the triplet excitons formed directly on the fluorescent dopant or transferred indirectly to the fluorescent dopant should be minimized. To solve this problem, an extremely low fluorescent dopant concentration is required which can reduce the direct exciton trapping by the dopant and suppress the short-range Dexter energy transfer process from $\mathrm{T}_{1}$ of the exciplex host to $\mathrm{T}_{1}$ of the dopant. In addition, designing appropriate HOMO and LUMO of both the host and dopant can also restrain the dopant from trapping excitons. ${ }^{114}$ Therefore, full exciton utilization and high EQE can be achieved for fluorescent OLEDs by adopting an exciplex host strategy.

\subsection{Sensitized fluorescence}

High-EQE OLEDs can be obtained by using TADF and phosphorescent emitters. However, the poor stability of blue TADF and phosphorescent OLEDs is still an unsolved problem. Blue fluorescent OLEDs have long operational lifetime but the EQE is limited to about $5 \%$ due to low exciton utilization. (a)

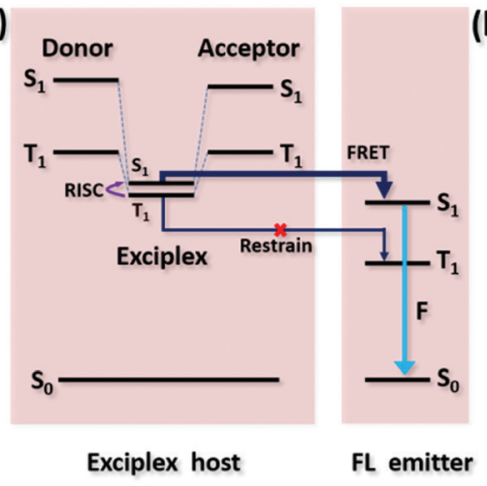

(b)

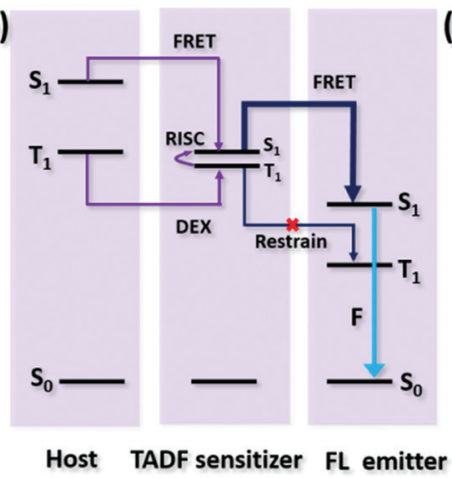

(c)

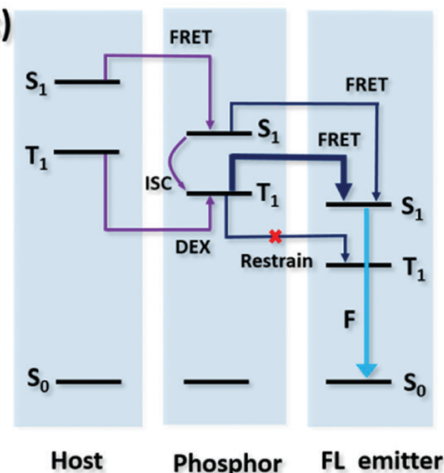

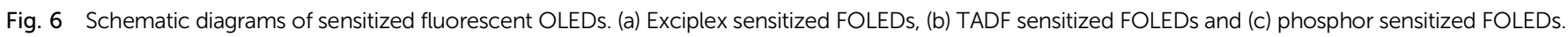


Fortunately, the low exciton utilization of conventional fluorescent OLEDs can be enhanced by a sensitized approach. ${ }^{114,121-127}$ Fig. 6 shows the schematic diagrams of sensitized fluorescent OLEDs; the sensitizer can be an exciplex, TADF emitter, and phosphor. In the previous section, we have discussed exciplex sensitized fluorescence OLEDs (Fig. 6a). However, blue OLEDs require a bluer emission spectrum of the exciplex. At the same time, the bluer exciplex requires a larger offset between the HOMO of the donor material and the LUMO of the acceptor material, and the emission energy of both the donor and acceptor should be larger. Therefore, this method is not suitable for blue OLEDs due to the rare blue exciplex.

For TADF sensitized fluorescent OLEDs, the photophysical process and required conditions are similar to those of an exciplex. The FRET process of excitons from $S_{1}$ of TADF to $S_{1}$ of the fluorescent emitter is the main energy transfer channel and the Dexter energy transfer process should be restrained (Fig. 6b). In addition to the conditions mentioned in the previous section, the TADF emitter should be able to have a large doping concentration of $15 \mathrm{wt} \%$ to $100 \mathrm{wt} \%$. By using this approach, Adachi and co-workers reported a series of highefficiency fluorescent OLEDs with EQE from $13.4 \%$ to $18 \%$ in the full spectrum range. In particular, a sensitized orange OLED showed a longer operation time compared to a pure TADF OLED. ${ }^{121}$ Moreover, Duan and co-workers reported another approach to inhibit the short-range Dexter energy transfer by modifying traditional fluorescent dopants with electronically inert terminal-substituents. The sensitized OLED exhibited an excellent EQE of $24 \%$ and a low-efficiency roll-off by combining with an interfacial exciplex. ${ }^{125}$

Due to the strong spin-orbit coupling effect, phosphorescent materials can emit light by triplet excitons and promote the ISC process from $S_{1}$ to $T_{1}$. Therefore, unlike TADF sensitized fluorescent OLEDs, the energy transfer of phosphor sensitized OLEDs mainly follows the route from $T_{1}$ of phosphor to $S_{1}$ of the fluorescent emitter by FRET (Fig. 6c). Based on such a strategy, a high-efficiency sky-blue fluorescent OLED with a long T80 of $320 \mathrm{~h}$ was obtained by Lovrincic and co-workers in 2018. ${ }^{126}$ In summary, developing sensitized fluorescent OLEDs is a possible approach to obtain efficient and stable blue OLEDs.

\section{Light extraction technologies}

\subsection{Light extraction losses}

As mentioned above, significant progress has been made in improving the internal quantum efficiency (IQE) to a high level of $\sim 100 \%$ with the full use of both singlet and triplet states. Unfortunately, most of the emitted photons are trapped within the device due to the optical confinement and internal reflection by the mismatched refractive index of the multilayer structure. ${ }^{128,129}$ The fraction of the extracted light to the air $\eta_{\text {out }}$ can be estimated based on ray optics according to Snell's law:

$$
\eta_{\text {out }}=\frac{1}{2 n^{2}}
$$

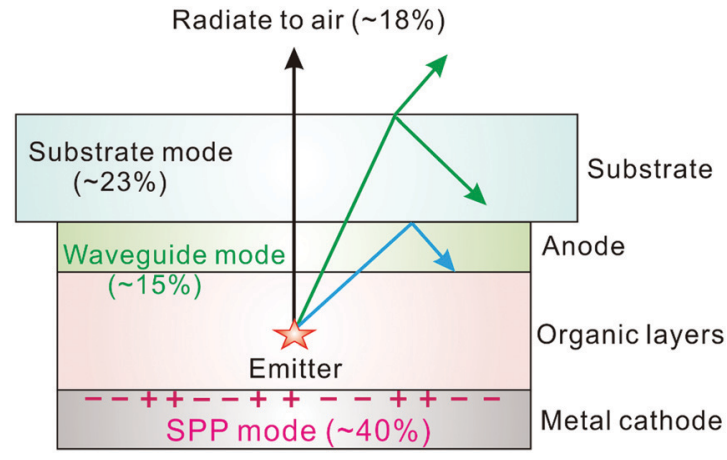

Fig. 7 Schematic illustration of various kinds of optical loss channels in conventional flat architectures.

Here $n$ depicts the effective refractive index of the organic layer stack with respect to the air space. Thus, the calculated extraction of light to the free space is only about $17 \% .{ }^{130,131}$ To gain further insight into optical loss channels, a more accurate treatment according to wave optical methods has been extensively investigated. As depicted in Fig. 7, an excited molecule can couple into different optical modes in such a flat stack structure. A significant portion of emitted photons are confined within the substrate (substrate mode, $\sim 23 \%$ ), ${ }^{132}$ guided within the organic layers and transparent anode (waveguide mode, $\sim 15 \%)^{130,133}$ and dissipated at the organic-cathode interface (SPP mode, $\sim 40 \%$ ), ${ }^{134,135}$ resulting in only $\sim 18 \%$ of energy flow being radiated outside the device as useful light. Thereby, the low-outcoupling efficiency limits highly efficient OLEDs. Accordingly, various advanced light out-coupling approaches have been extensively explored to recover the wasted photons for practical lighting and display applications in recent years, as will be discussed in detail.

\subsection{Microscale structures}

To date, various methods have been proposed to enhance the light extraction for OLEDs. For this purpose, particular structures are desirable to effectively decrease the incident angles at the interfaces, and thus avoid the total internal reflection of emitted light. From the point of view of ray optics, microscale structures constructed on device substrates can efficiently suppress the substrate mode loss. Möller and Forrest incorporated a poly-dimethyl-siloxane (PDMS) microlens array (MLA) film with a diameter of $10 \mu \mathrm{m}$ onto a glass substrate as a light extraction film, which was produced by photolithographic techniques and a mold transfer process. ${ }^{136}$ The EQE of OLEDs with MLAs was enhanced by a factor of at least 1.5, and no color changes of the emission were observed. Later, Helander and co-workers reported a double layer green OLED with a maximum PE of $230 \mathrm{~lm} \mathrm{~W}^{-1}$ by combining a chlorinated ITO cathode and the MLAs. ${ }^{137}$ Yang and co-workers demonstrated an enhancement of the light extraction efficiency by incorporating three differently sized MLAs onto a glass substrate, which were fabricated with the employment of a roll-to-roll mold transfer process on polycarbonate (PC) films (Fig. 8a). ${ }^{138}$ Compared to the flat device, the light extraction enhancement ratios of devices with 

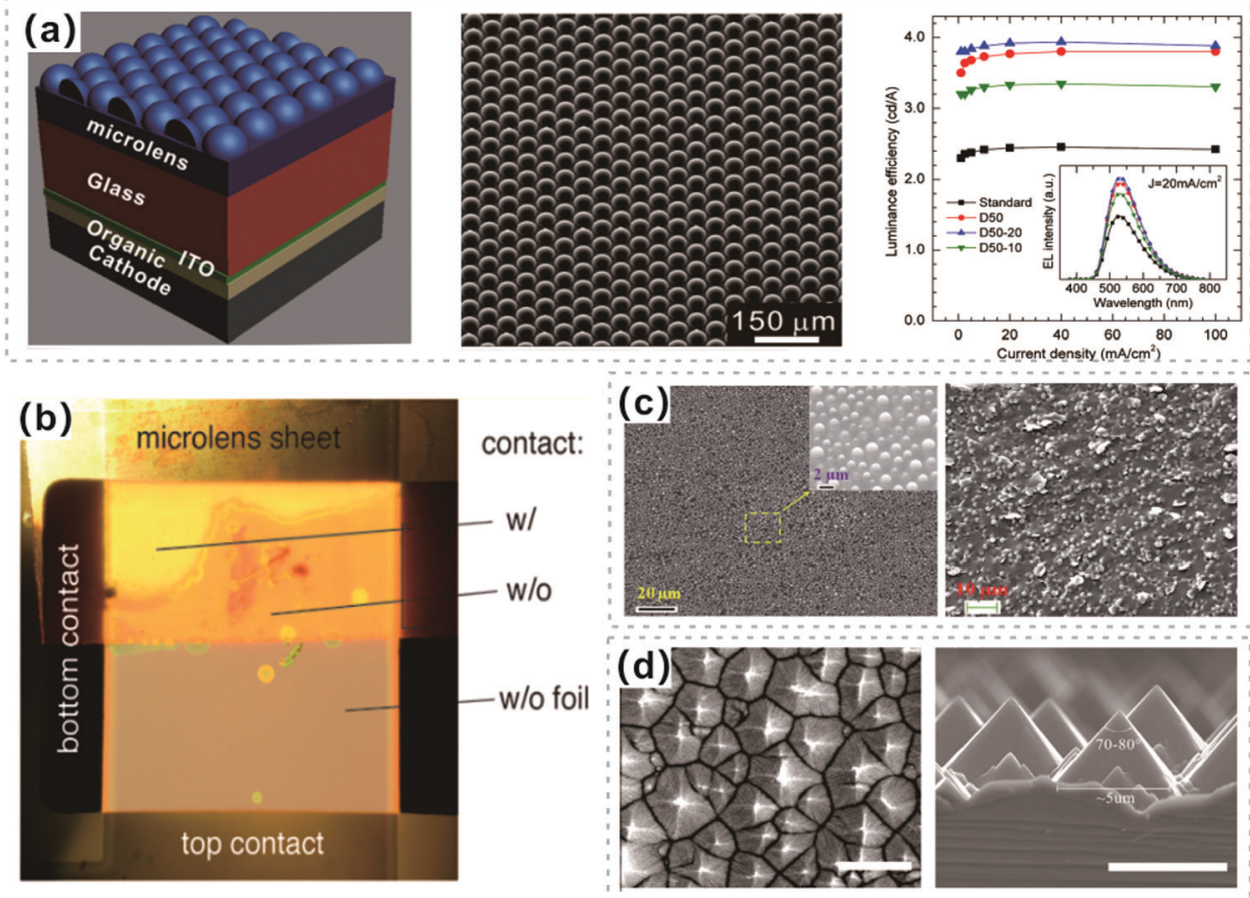

Fig. 8 (a) (left) An OLED attached with MLAs on a glass substrate as a light extraction film, (middle) the $45^{\circ}$ tilted view of the SEM image of MLAs with a hemispherical shape, and (right) luminance efficiency as a function of current density inserted with EL spectra of devices with and without MLAs. Reproduced with permission from ref. 138. Copyright 2010 American Institute of Physics. (b) Photography of a top-emission OLED that is only partially covered with a MLA film. Reproduced with permission from ref. 139. Copyright 2011 American Chemical Society. (c) Top-view and cross-section SEM images of a microscale sphere-based outcoupling layer. Reproduced with permission from ref. 140. Copyright 2014 Royal Society of Chemistry. (d) Top-view and cross-section SEM images of micro-pyramids of a PVA film. Reproduced with permission from ref. 141. Copyright 2005 Elsevier B.V.

various MLAs were $1.52,1.60$, and 1.36 for D50, D50-20, and D50-10 $(50 \mu \mathrm{m}$ in diameter, referred to as D50, and $50 \mu \mathrm{m}$ in diameter and $20 \mu \mathrm{m}$ in transverse diameter, referred to as D50-20) in the normal direction, respectively. Similarly, a negligible color shift for OLEDs with the MLA film was observed in this device configuration. Thomschke and co-workers used a thick optical cavity with a refractive index matched microlens foil as an integrating element on top-emitting WOLEDs, yielding a 4-fold increase of spectral enhancement and a record high CRI of 93 for top-emitting WOLEDs (Fig. 8b). ${ }^{139}$ Theoretical and experimental investigations demonstrated the light out-coupling ability and the integrating ability of the microlens foils, which mix the optical modes to a broadband spectrum.

In addition, the introduced random-distributed microscale structures can also efficiently release the trapped photons in OLEDs. Liu and co-workers developed a flexible light extraction film, which consists of a polyethylene naphthalate (PEN) substrate and randomly distributed $\mathrm{SiO}_{2}$ spheres (refractive index of $\sim 1.5$, an average particle size of $1.5 \mu \mathrm{m}$ ) in a SU-8 matrix (Fig. 8c). ${ }^{140}$ These embedded spheres can efficiently scatter and redirect the emitted light, thus leading to the efficiency improvement of the OLED devices. As a consequence, the fabricated flexible WOLED on the PEN substrate with a thin encapsulation film and a light extraction film exhibited a lifetime of $5.2 \mathrm{~h}$ at an initial luminance of $1000 \mathrm{~cd} \mathrm{~m}^{-2}$ and the maximum power efficiency exceeded $100 \mathrm{~lm} \mathrm{~W}^{-1}$ with negligible CIE variation. Except for commonly used MLAs and sphere particles, micro-pyramid structures were also investigated. Zhu and co-workers duplicated the microscale pyramid-based texture from a monocrystalline silicon surface to a polyvinyl alcohol (PVA) film using a printing process and applied it to the surface of OLEDs with the spires of pyramids facing the emitter (Fig. 8d). ${ }^{141}$ It was observed that this stratagem could effectively enhance light escape, while the CE and EQE got enhancement ratios of $37.3 \%$ and $36.6 \%$, respectively, at a current density of $100 \mathrm{~mA} \mathrm{~cm}{ }^{-2}$ without inducing any side effects. To sum up, the conventional substrate-mode light loss in OLEDs can be effectively weakened by means of microscale structure light extraction schemes.

\subsection{Nanoscale structures}

Another way of guiding the trapped light to air is the use of nanostructures based on the theory of wave optics, i.e. onedimensional (1D) nanograting, random nanostructures, and nanoscale particles. A few representative structures will be discussed in this section.

4.3.1. Periodic nanostructures. Periodic Bragg nanograting has been introduced into OLEDs for light extraction by Lupton et al. and Matterson et al. ${ }^{142,143}$ They have demonstrated enhanced light extraction by using a nanostructured photoresist layer, which introduces Bragg-scattering of the waveguided light. Nevertheless, the absorptive gold and photoresist layers in this architecture limited the absolute efficiency of OLEDs. ${ }^{144}$ Later, Ziebarth and co-workers incorporated periodic nanogratings 
into a poly(3,4-ethylenedioxythiophene)-poly(styrenesulfonate) (PEDOT:PSS) layer using soft nanoimprint lithography (SNIL) technology in order to fabricate more conventional ITO-based devices. ${ }^{145}$ However, the waveguide absorption in ITO and PEDOT:PSS results in a suppressed grating effect especially in the shorter wavelength region. A schematic illustration of the Bragg diffraction process of waveguided light in periodic nanograting structures is displayed in Fig. 9a. ${ }^{146}$ It is noted that the emission angle of extracted light $\left(k_{0}\right)$ depended on the Bragg equation. In particular, the emission angle is governed by the conservation of momentum in the plane of the waveguide, which is modulated by the parameters of wavelength $(\lambda)$, the wavenumbers of the waveguided light $\left(k_{\mathrm{wg}}\right)$, and the grating $\left(k_{\mathrm{g}}\right)$. This means that the peak splitting of the EL spectra must occur because the diffracted waveguide light travels in the opposite direction. Tang and co-workers proposed a scheme for incorporating double-pattern Bragg gratings in organic layers to enhance the light extraction of the trapped optical modes in polymer light-emitting diodes (PLEDs) (see Fig. 9b). ${ }^{147}$ They observed that the resulting current efficiency of the double-pattern
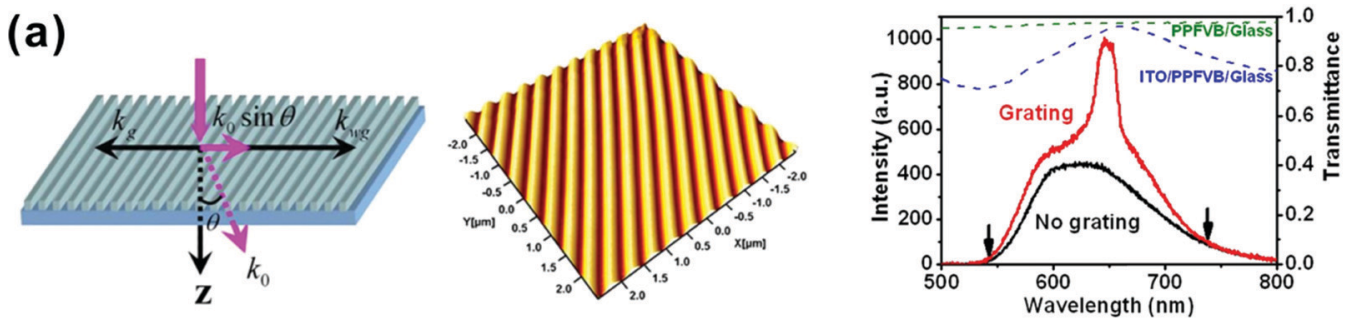

(b)

(c)
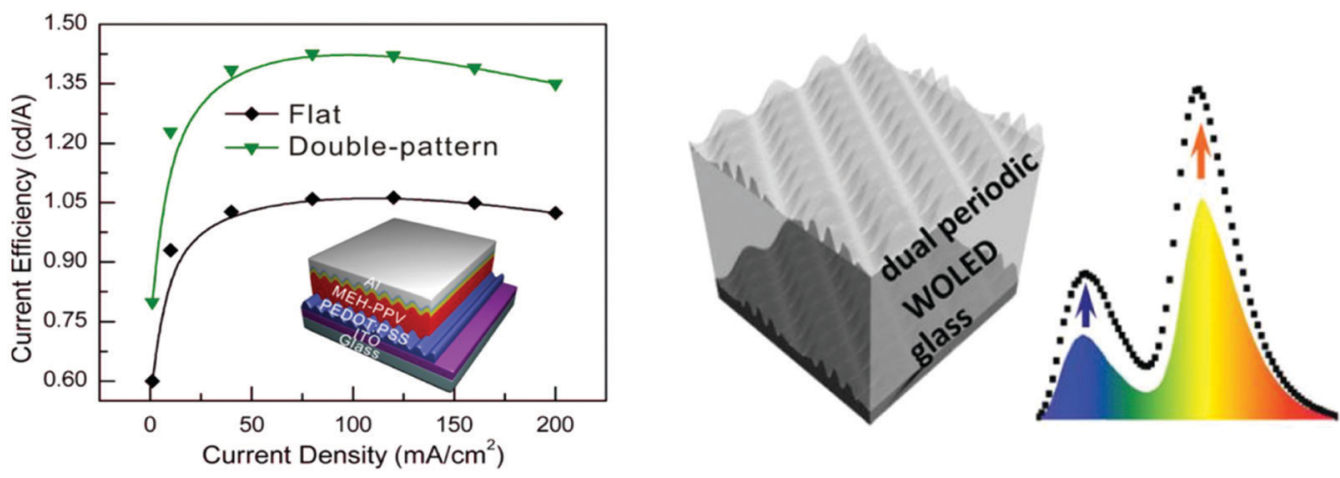

(d)

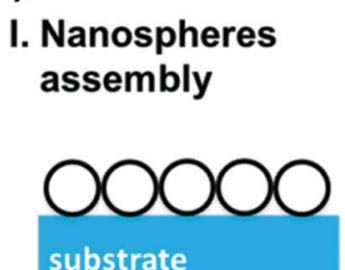

IV. Lift-off

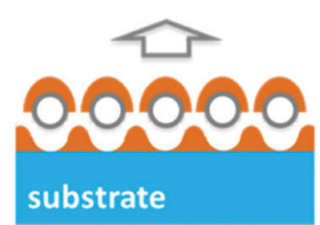

II. RIE etching

Plasma

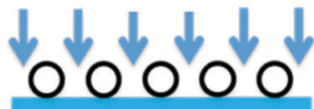

substrate

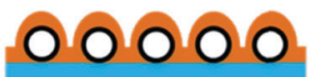

substrate
III. Sputter ITO

VI. Complete device

V. PEDOT:PSS

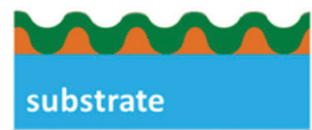

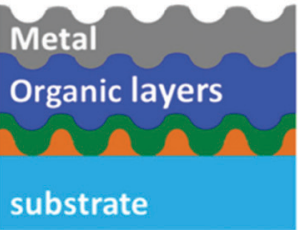

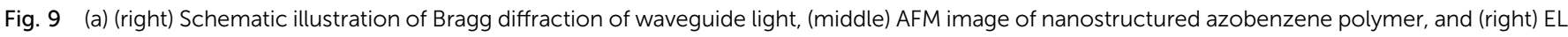

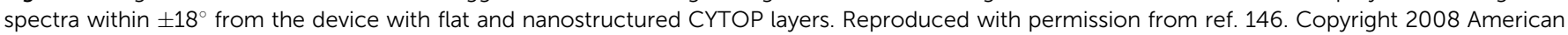

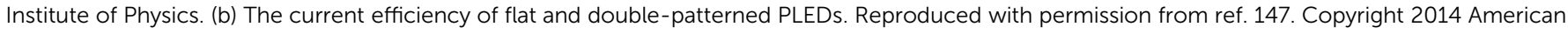

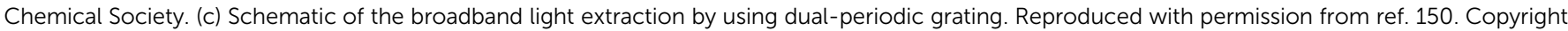

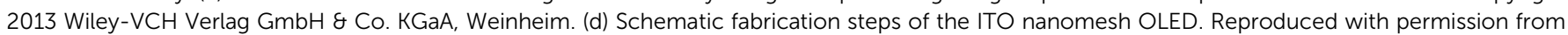
ref. 151. Copyright 2015 Wiley-VCH Verlag GmbH \& Co. KGaA, Weinheim. 
device was 1.35 times that of the conventional device with a flat architecture. This corrugated structure-enhanced light outcoupling efficiency was attributed to the combination of the ordinary Bragg scattering, guide-mode resonance (GMR), periodic interference modulation, surface plasmon polariton modes (SPP) and the hybrid anticross coupling between GMR and SPP. However, these diffraction gratings enhance the outcoupling of the waveguided light only at specific emission wavelengths, giving rise to difficulties in WOLED applications. ${ }^{148,149}$

Sun and co-workers illustrated broadband excitation and outcoupling of the trapped photons within the WOLEDs by introducing a two-dimensional (2D) grating with dual-periodic corrugation into the devices (Fig. 9c). ${ }^{150}$ This dedicatedly designed structure consisting of two sets of corrugations with different periods that can broaden the light was directly amenable to extracting light from the WOLEDs based on two complementary color strategies of blue and orange emission. As a consequence, a $48 \%$ enhancement of EQE was obtained compared to that of the conventional planar devices. Wu and co-workers reported a novel internal extraction structure toward high OLED efficiency by the formation of an ITO nanomesh and its filling of high-conductivity PEDOT:PSS. ${ }^{151}$ As depicted in Fig. 9d, such a structure can be readily fabricated by facile nanosphere lithography (of ITO) and spin-coating (of PEDOT:PSS), by which the EQE of OLEDs is enhanced by a factor of $41 \%$. Considering the different refractive indices of ITO ( $n=1.9-2.1)$ and PEDOT:PSS $(n=1.5)$, as well as the incomplete planarization of the ITO nanomesh, they suggested that such a significant enhancement of OLED efficiency originated from the synergetic effect of photonic crystals and surface corrugation. Therefore, employing the periodic nanograting/mesh in OLEDs has the potential to fabricate high-performance devices for commercial applications of lighting and displays.

4.3.2. Aperiodic nanostructures. The utilization of aperiodic nanostructures for light out-coupling in OLEDs is a fundamentally different approach as compared to the periodic nanostructures. Koo and co-workers fabricated corrugated green OLEDs based on a buckling patterned PDMS/Al film which was constructed on a glass substrate. ${ }^{152}$ Buckling patterns were spontaneously formed on PDMS due to the difference in the thermal expansion coefficients between PDMS and the thin metallic Al film. AFM images and the corresponding fast Fourier transform (FFT) pattern showed that the buckling structure has a characteristic wavelength with a wide distribution and without preferred orientation. The CE and PE of the corrugated devices at $2000 \mathrm{~cd} \mathrm{~m}^{-2}$ were improved by a factor of 1.8 and 2.2, respectively, with double buckling, which is 2.2 and 2.9 for the devices with triple buckling. Meanwhile, the devices with buckling show higher current density and luminance than flat devices. These improved $J-V$ properties are attributed to the stronger electric field between the peak and valley of the sinusoidal patterned structures. ${ }^{153-155}$ Zhou and co-workers proposed a method of efficient photo flux manipulation by introducing bio-inspired moth's eye nanostructures (MENs) into OLEDs on ITO-glass substrates. ${ }^{107}$ Light-coupling efficiency of OLEDs with stacked triple emission units was over
2 times that of a conventional device, resulting in a drastic increase in EQE and $\mathrm{CE}$ to $119.7 \%$ and $336 \mathrm{~cd} \mathrm{~A}^{-1}$ without introducing spectral distortion and directionality. Similarly, $\mathrm{Ou}$ and co-workers reported an efficient WOLED structure combining MENs for broadband quasi-omnidirectional light outcoupling and a multilayer energy cascade structure for energy-efficient photon generation (Fig. 10a). ${ }^{156}$ The EQE and PE of the optimized device with MENs were increased to 54.6\% and $123.4 \mathrm{~lm} \mathrm{~W}^{-1}$, respectively, at $1000 \mathrm{~cd} \mathrm{~m}^{-2}$. Moreover, they observed that this OLED structure offered an extremely small roll-off in efficiency at high luminance, yielding a striking value of $106.5 \mathrm{~lm} \mathrm{~W}^{-1}$ at $5000 \mathrm{~cd} \mathrm{~m}^{-2}$. To further investigate the light extraction capability, Zhou and co-workers demonstrated an interesting method for releasing the trapped energy flow in conventional WOLEDs by implementing inverted MENs via SNIL technology (Fig. 10b). ${ }^{157}$ The resulting efficiency is over 2 times that of a conventional OLED, which provided direct evidence that inverted MENs can afford identical outcoupling capability as compared with MENs. Besides, MENs constructed on glass substrates outside the device afford an enticing capacity against scrubbing and a self-cleaning feature, which benefits the commercial viability in practical applications. Most recently, an effective way to extract waveguide-mode light has been reported by Reineke and co-workers. ${ }^{158}$ In their work, controllable quasiperiodic nano-microstructures with a broad periodicity and depth distribution in the range from tens of nanometers to micrometers are used, which are induced by reactive ion etching (RIE) on poly(dimethylsiloxane) (PDMS) surfaces (Fig. 10c). Consequently, the external quantum efficiency of the ITO-based two-unit tandem white OLEDs can be enhanced by a factor of $1.53 \pm 0.12$ at $10000 \mathrm{~cd} \mathrm{~m}^{-2}$, without introducing angular or wavelength-dependent emission. Hence, aperiodic nanostructured OLEDs have the advantage of a broad distribution in periodicity and random directionality, allowing the modes guided in all azimuthal directions to be extracted over the entire emission wavelength range.

In addition, many studies concerning OLEDs utilizing nanoparticles (NPs) have been performed to enhance light escape. Yang et al. have demonstrated SPP-enhanced OLEDs by introducing silver NPs. ${ }^{159}$ As shown in Fig. 11a, Gu et al. reported a $\sim 123 \%$ enhancement of current efficiency (with a current density of $\left.20 \mathrm{~mA} \mathrm{~cm}{ }^{-2}\right)$ by introducing platinum-cobalt $\left(\mathrm{Pt}_{3} \mathrm{Co}\right)$ alloy nanoparticles into devices. ${ }^{160}$ Grover and co-workers have proposed a light extracting method of incorporating a crystallized 4,7-diphenyl-1,10-phenanthroline (BPhen) film as a scattering layer at the backside of an ITO coated glass substrate. ${ }^{161}$ In Lee and co-worker's research, a $200 \%$ enhancement of PE has been achieved by using corrugated PEDOT:PSS cross-linked PS NPS as the HTL, which simultaneously reduces the optical loss and alleviate degradation of devices (Fig. 11b). ${ }^{162}$ Kim and co-workers reported performance enhancement of devices by utilizing a poly(4-butylphenyl-diphenyl-amine) (poly-TPD):dodecanethiol functionalized $\mathrm{Au}$ (DDT-Au) nanocomposite layer (Fig. 11c). ${ }^{163}$ Later, they observed that the enhanced EL intensity for the device with a nanocomposite layer was strongly related to the effective energy transfer between the radiated light and the LSPR 
(a)

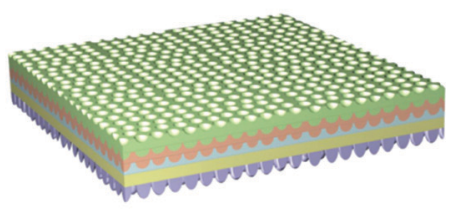

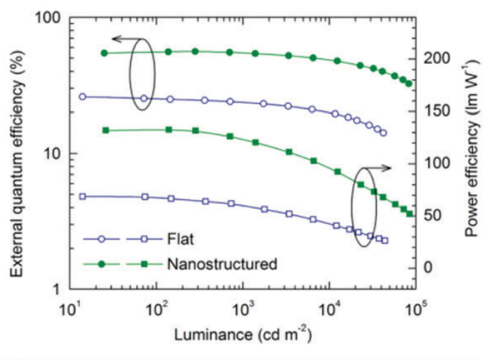

(b)

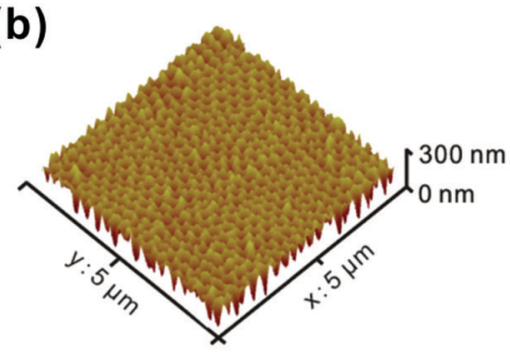

(c)

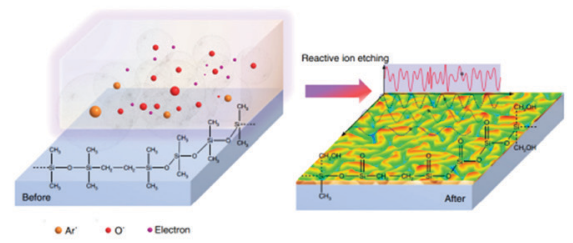

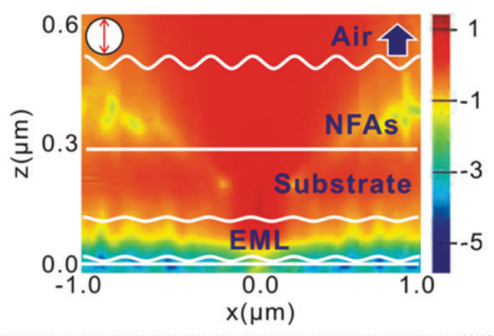

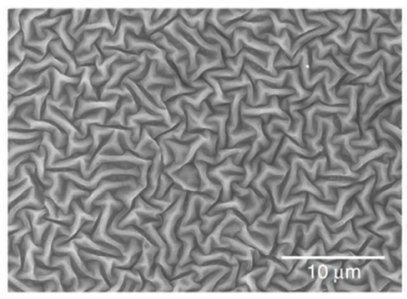

Fig. 10 (a) Schematic of WOLEDs, in which both sides of the ITO glass substrate are patterned with MENs, along with EQE and PE as a function of luminance for the corresponding devices. Reproduced with permission from ref. 156. 2014 Wiley-VCH Verlag GmbH \& Co. KGaA, Weinheim. (b) AFM images of patterned inverted MEN UV-resin on the glass surface, and calculated electric field distributions at $520 \mathrm{~nm}$ with a single perpendicular-oriented dipole located in the middle of the emission layer for the corrugated device. Reproduced with permission from ref. 157. 2015 Wiley-VCH Verlag GmbH \& Co. KGaA, Weinheim. (c) Schematic illustrations of RIE-induced nanostructures and relevant SEM images. Reproduced with permission from ref. 158. Copyright 2019 Macmillan Publishers Limited.

excited by the DDT-Au NPs (Fig. 11d). ${ }^{164}$ Recently, a novel nanocomposite substrate consisting of a silver nanowire-based electrode, a high-index polymer layer, and a light-scattering layer has been demonstrated by Pei and co-workers (Fig. 11e). ${ }^{165}$ The high-index polymer $(n=1.7)$ was employed to improve the refractive index matching of the substrate and the organic layers ( $n=1.7-1.8)$. The composite light scattering layer, comprising an acrylate admixed with high-index barium strontium titanate nanoparticles, is designed to extract waveguide-mode and substrate-mode light. Based on these versatile substrates, efficient white OLEDs with a PE of $107 \mathrm{~lm} \mathrm{W^{-1 }}$ and an EQE of $49 \%$ at $1000 \mathrm{~cd} \mathrm{~m}^{-2}$ have been subsequently fabricated. The influence of refractive indices of various components in OLEDs on light extraction will be discussed in the next section.

4.3.3. Refractive index modulation layer. The refractiveindex values of different media in OLEDs significantly influence the inside light-transmitting behavior and are thus related to the consequent light-emitting performance. Quite a part of light loss in OLEDs results from the total internal reflection of light at interfaces, which is induced by refractive-index differences between layers. The use of a nanostructured refractive index modulation layer enables the confined light to be emitted into the air; as a consequence, the outcoupling efficiency is enhanced. Forrest and co-workers incorporated a low index grid $\left(\mathrm{SiO}_{2}, n=1.5\right)$ at the interface of ITO and the organic layer for light out-coupling. ${ }^{166}$ The embedded low-index grid enables the waveguided light to be released into glass and air modes. By using the MLAs, the resulting out-coupling efficiency was 2.3 times greater than that of a conventional flat device. Recently, they demonstrated an efficient top-emitting OLED benefitting from a versatile bottom electrode/substrate structure (Fig. 12a). ${ }^{167}$ Such a structure is integrated with a transparent layer of ITO deposited on a polytetrafluoroethylene (PTFE, Teflon) diffuse reflector, which is planarized by a thick polymer slab waveguide. Since this light-scattering dielectric layer eliminates plasmonic, waveguide, and substrate-mode light loss, the external quantum efficiency is increased from $15 \pm 2 \%$ to $37 \pm 4 \%$. As depicted in Fig. $12 \mathrm{~b}$, Yoo et al. proposed a simple and cost-effective method for releasing substratemode and waveguide light by using industrial-grade and high refractive-index polyethylene naphthalate (PEN) as a substrate. ${ }^{168}$ The high refractive index of PEN substrates is further combined with the built-in scattering properties of their industrial-grade characteristics. Furthermore, a planarization layer was constructed by coating a thick film of polyamide-imide (PAI) with 


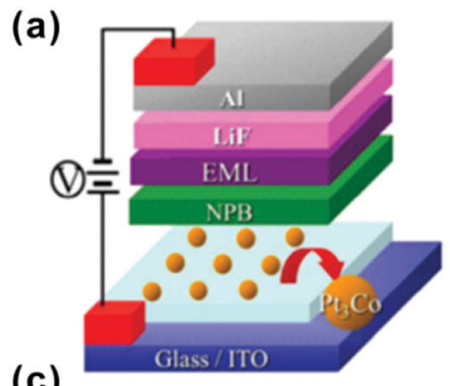

(c)

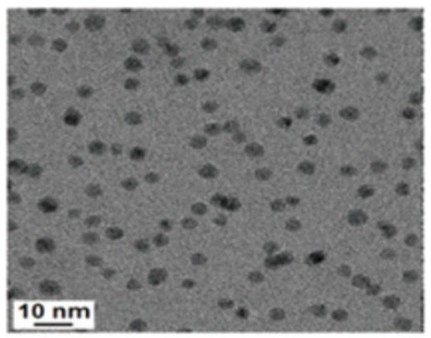

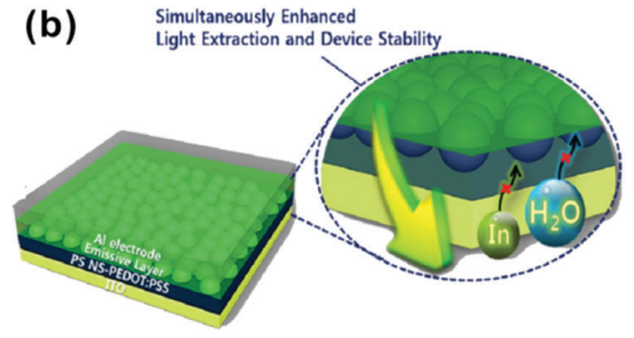

(d)

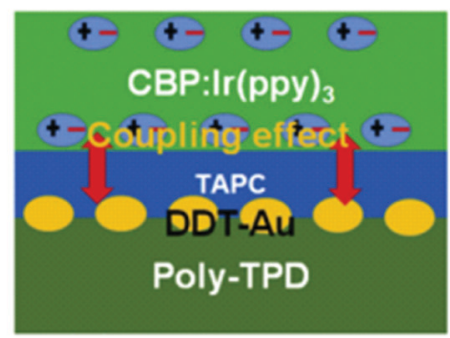

(e)

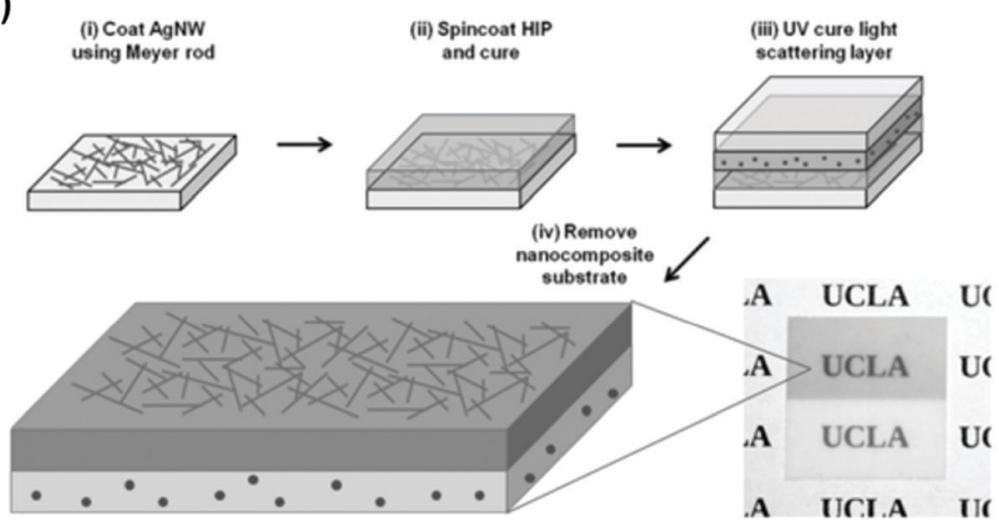

Fig. 11 (a) Schematic device structure of an OLED incorporating Pt ${ }_{3}$ Co ANPs on an ITO anode. Reproduced with permission from ref. 160. Copyright 2013 Royal Society of Chemistry. (b) Device structure with polystyrene (PS) nanospheres embedded in PEDOT:PSS films. Reproduced with permission from ref. 162. 2014 Wiley-VCH Verlag GmbH \& Co. KGaA, Weinheim. (c) The TEM image of the poly-TPD:DDT-Au nanocomposite film. Reproduced with permission from ref. 163. Copyright 2015 Optical Society of America. (d) Device structure with a DDT-Au layer. Reproduced with permission from ref. 164. Copyright 2016 Elsevier B. V. (e) Fabrication process for a freestanding nanocomposite substrate containing silver nanowires embedded in the high-index polymer (HIP) layer and barium strontium titanate (BST) nanoparticles embedded in the light-scattering layer. Reproduced with permission from ref. 165. Copyright 2017 Wiley-VCH Verlag GmbH \& Co. KGaA, Weinheim.

a refractive index of 1.7 for better refractive index matching between PEN and the electrode. An EQE enhancement ratio of as high as 1.76 based on the PEN-substrate scheme has been achieved. Apart from the refractive index modulation of substrates, lowering the refractive index of the electron transport layer (ETL) also holds great potential for light extraction, since it is the layer adjacent to the metallic cathode. On this issue, Shi $e t$ al. recently reported an oblique angle deposition (OAD) method to manipulate the refractive index of tris(8hydroxyquinoline) aluminum (Alq3) by creating an ordered columnar void structure (Fig. 12c). ${ }^{169}$ By tuning the refractive index of $\mathrm{Alq}_{3}$ from 1.75 to 1.45, the SSP-mode light loss is significantly reduced, which has been revealed by optical simulation and experimental results. These proposed refractive index modulation layers are also very promising to steering light in other optoelectronic devices.

\subsection{Multiscale structures}

Recently, multiscale hierarchical structures have attracted much attention and inspired highly efficient light management approaches. ${ }^{170-173}$ As displayed in Fig. 13a, Zhou and co-workers fabricated multiscale micro-nano nested structures featuring a microlens and microtriangle array with built-in nanohole structures (MTNs) by combining the mask photolithography and controllable thermal reflow process. ${ }^{174}$ As a result, the device with MTNs constructed on glass substrates exhibited reduced reflection of $7.1 \%$ over the entire visible wavelength range, which is $3.5 \%$ lower than the bare substrate. The resulting CE and EQE were 1.60 and 1.63 times that of a flat architecture. Most recently, inspired by the multiscale structure of the firefly's cuticle, Jeong and co-workers developed a new method to fabricate this hierarchical ultrastructure using geometry-guided resist reflow, replica molding, and PDMS oxidation. ${ }^{175}$ These newly developed 
(a)

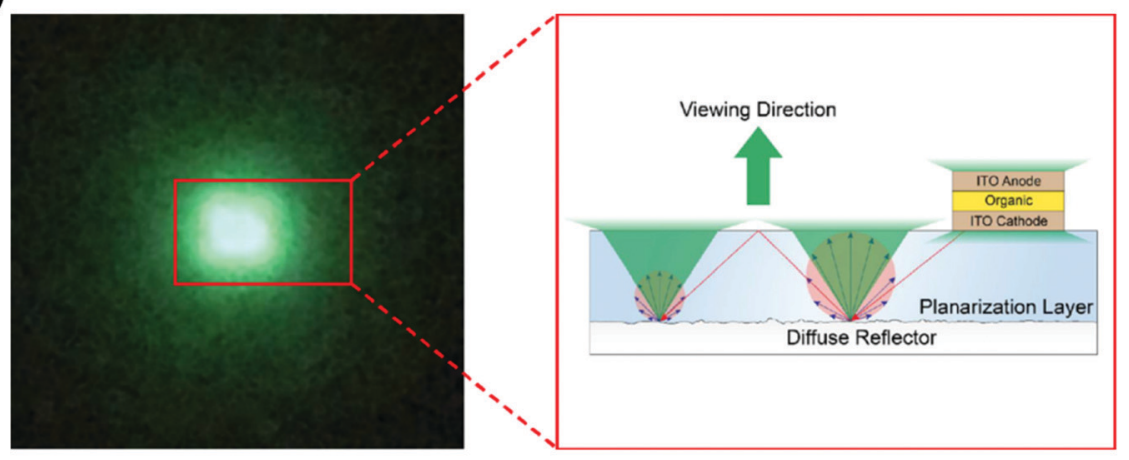

(b)
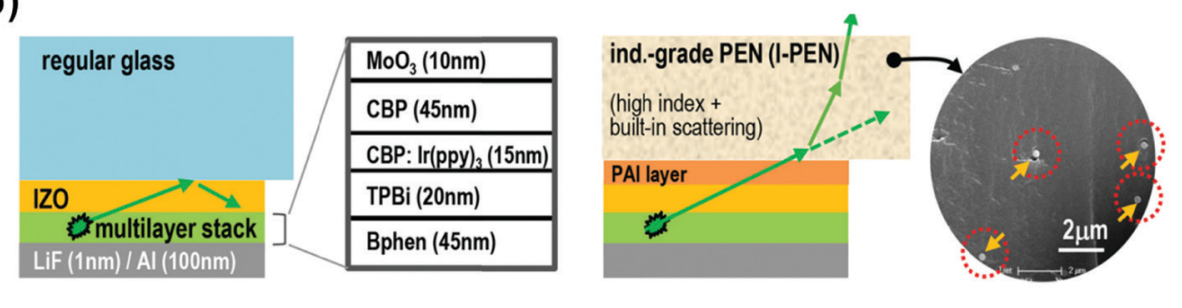

(c)
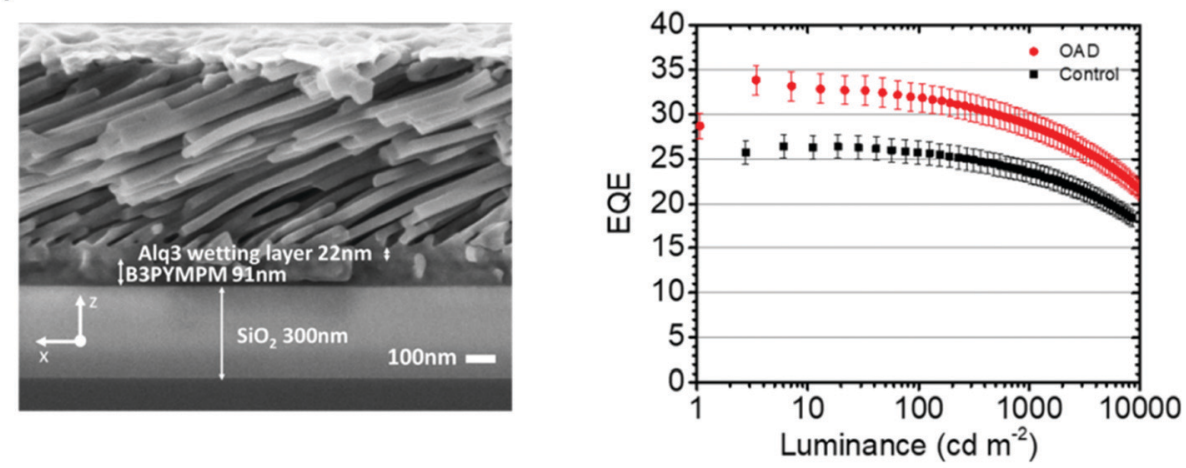

Fig. 12 (a) Schematic illustration of the OLED on a diffuse reflector. Reproduced with permission from ref. 167. Copyright 2018 American Chemical Society. (b) Schematic diagrams of OLED structures on regular glass substrates (left) and on industrial-grade PEN (I-PEN) substrates (right). Reproduced with permission from the ref. 168. Copyright 2015 Wiley-VCH Verlag GmbH \& Co. KGaA, Weinheim. (c) Cross-sectional SEM image of $\sim 600$ nm of Alq 3 deposited at $80^{\circ}$ on top of $90 \mathrm{~nm}$ B3PYMPM deposited at $0^{\circ}$ on top of $300 \mathrm{~nm}$ of $\mathrm{SiO}_{2}$ and EQE values of various OLEDs. Reproduced with permission from ref. 169. Copyright 2018 American Chemical Society.

hierarchical structures were composed of longitudinal nanostructures and asymmetric microstructures (Fig. 13b), which can efficiently extract the photons confined in the OLED due to total internal reflection. The introduction of hierarchical structures leads to a $60 \%$ increase in the EQE and an illumination angle $16 \%$ wider than that of the flat device. As displayed in Fig. 13c, a glass-fabric reinforced siloxane hybrid (GFRH) substrate has been demonstrated as a facile substrate of OLEDs for extracting light trapped in substrate mode. ${ }^{176}$ In order to enhance light scattering inside the substrate, the refractive index of the siloxane hybrid was rationally modified to maximize the scattering of light at the interface of the matrix and the glass fabric, resulting in a film with high optical transmittance and haze. As a consequence, a high-performance OLED was successfully fabricated on the film and exhibited a $74 \%$ enhancement of EQE owing to the outstanding optical properties of the
GFRH film. These newly emerging multiscale structures will set a new paradigm for light extraction engineering for commercial lighting applications.

\subsection{Orientation of the molecular dipoles}

The orientation of emitting dipoles has been demonstrated to be one of the most important properties of emitters influencing the efficiency of OLEDs. Particularly, horizontally oriented emitting dipoles in OLEDs lead to reduced light losses from surface plasmon modes and waveguide modes, hence increasing the outcoupling efficiency of OLEDs. In 2009, Adachi and co-workers demonstrated some molecular design guidelines for highly horizontally oriented materials by assessing optical properties of typical organic materials. ${ }^{177}$ They observed the fact that horizontal molecular orientation occurs on various underlying layers, implying the versatility of the horizontal 
(a)

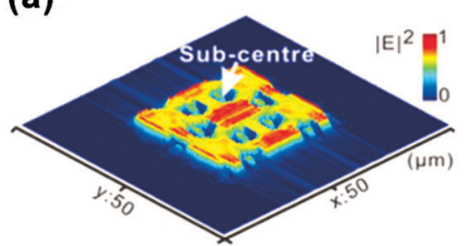

Fresnel diffraction

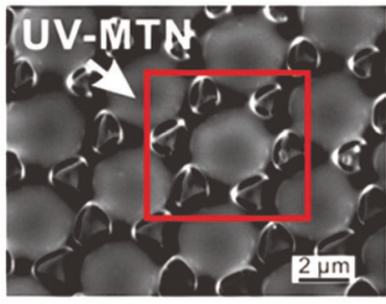

Multiscale micro-nano nested structures (b)

\section{Bioinspired OLED}

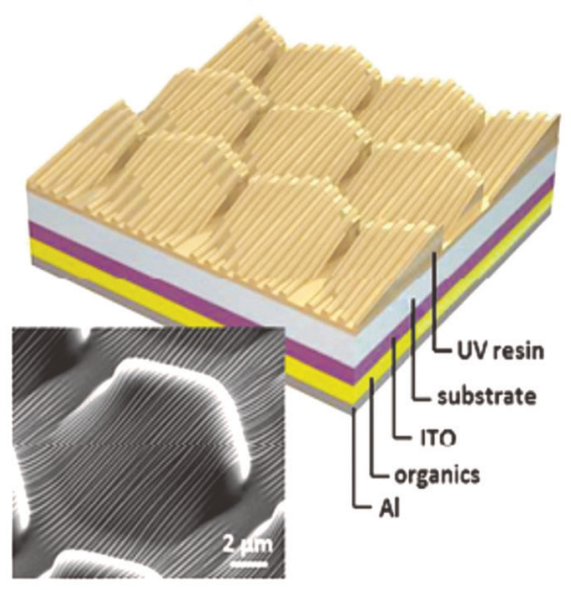

(c)
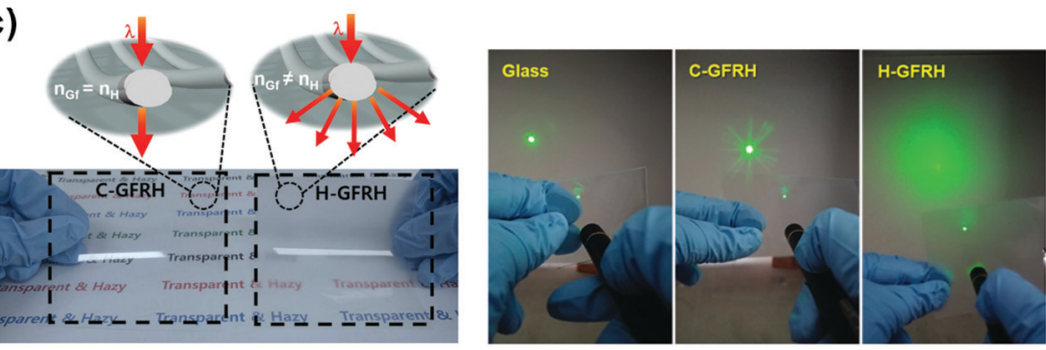

Fig. 13 (a) (top) Calculated normalized intensity distribution of the Fresnel diffraction image for the pre-designed mask. (bottom) SEM image of micronano nested structures featuring a microlens and micro triangle array with built-in nanohole structures. Reproduced with permission from ref. 174 . Copyright 2015 American Chemical Society. (b) The device structure of the bio-inspired OLED and the SEM image of the multiscale structures mimicking the hierarchical structures of a firefly lantern. Reproduced with permission from ref. 175. Copyright 2016 American Chemical Society. (c) Schematic illustration of the fabrication procedure for Ag-grid embedded GFRH films and light scattering by the refractive index contrast of the glass fiber and the hybrimer. The Ag grid was prepared by the gravure-offset printing method. Reproduced with permission from ref. 176. Copyright 2018 Wiley-VCH Verlag $\mathrm{GmbH} \& \mathrm{Co}$. KGaA, Weinheim.

orientation for various applications. Subsequently, Yokoyama and co-workers have systematically reviewed several factors of molecular orientation in vacuum deposited amorphous films of various OLED materials. ${ }^{178}$ The effect of the horizontal orientation on the light extraction of small-molecule OLEDs is schematically depicted in Fig. 14a. Since the photons were emitted mainly in the direction vertical to the transition dipole moment, the horizontal orientation was preferable for high light extraction efficiency. Frischeisen $e t$ al. presented an experimental method to straightforwardly determine dipole orientation in guest-host systems and found that light extraction was increased by around $45 \%$ when the majority of dipoles were horizontally orientated. ${ }^{179}$ It was observed that the typical green emitter of $\operatorname{Ir}(\mathrm{ppy})_{2}(\mathrm{acac})$ exhibited nearly $19 \%$ higher EQE than its $\operatorname{Ir}(\mathrm{ppy})_{3}$ counterpart. This feature is directly attributed to the completely isotropic behavior of $\operatorname{Ir}(\mathrm{ppy})_{3}$ and $77 \%$ horizontally oriented aligned dipoles of $\operatorname{Ir}(\mathrm{ppy})_{2}(\mathrm{acac}) .{ }^{180}$ By using the same emitter, Lu et al. reported a high-efficiency green phosphorescent OLED adopting both the low-index ITOfree electrode and the preferentially horizontal dipole emitter of $\operatorname{Ir}(\mathrm{ppy})_{2}(\mathrm{acac}) .^{181}$ The highest EQE of this proposed device at that time reached up to $64 \%$ with an MLA light extraction film (Fig. 14b). Though Ir-based complexes were reported having a horizontal orientation to improve light outcoupling, the internal mechanism had not been fully uncovered until Kim and co-workers' report. They found that the substituents at the para-position of the pyridine in the main ligands of Ir-based complexes play a vital role in changing the orientation of Ir-based complexes. ${ }^{182}$ They concluded that horizontal emitting orientation is highly possible if Ir-based complexes are elongated by substituting aliphatic or aromatic functional moieties at the para-position of the pyridine in the main ligands. By using this scheme, stacked Ir-based complexes with orientation parallel to the film surface are formed (Fig. 14c). They benefit from the elongated and planar substituents with high electrostatic potential which enlarges the interacting surface region between the Ir-based complex and host molecules. Apart from Ir-based phosphorescent emitters, TADF-based OLEDs have also been reported showing elevated efficiencies based on horizontally orientated emitting dipoles. For instance, Lee and co-workers demonstrated that a near-perfect $(100 \%)$ horizontal orientation of the TADF dipole moment can be induced by using triscarbazole donors (Fig. 14d). ${ }^{183}$ Furthermore, a triscarbazole-based emitting system possesses high PL quantum yield, which is due to the 
(a)

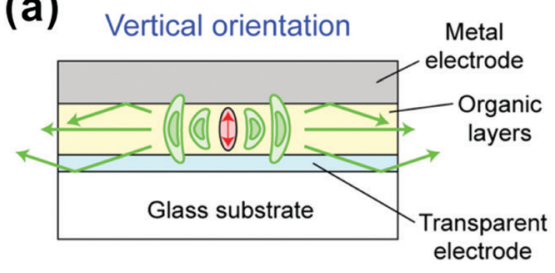

Horizontal orientation

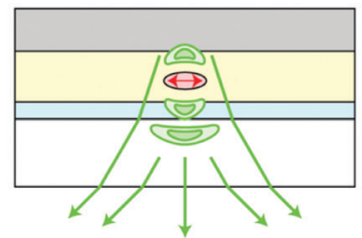

(c)

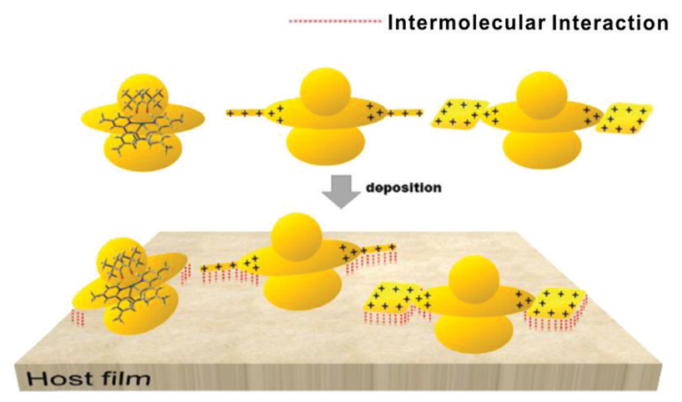

(b)

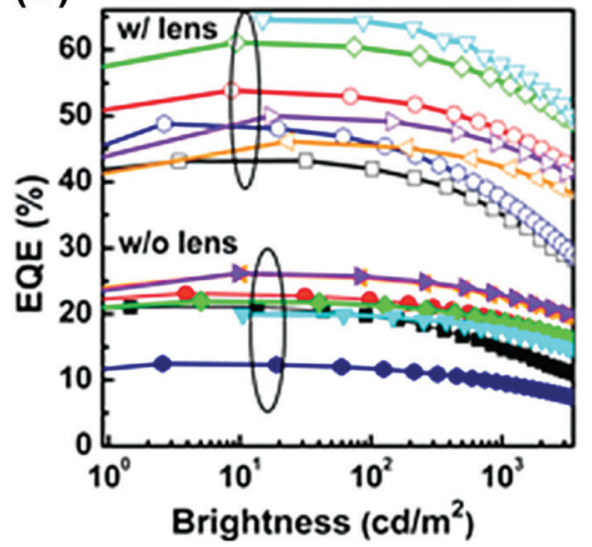

(d)

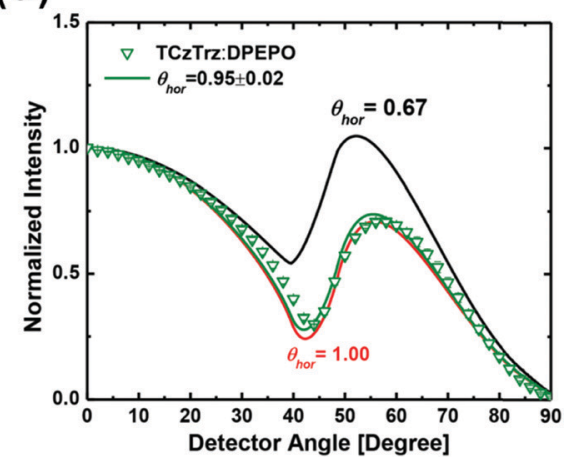

Fig. 14 (a) Schematic of the enhancement of outcoupling efficiency of OLEDs by the horizontal orientation of the transition dipole moment of emitting molecules. Reproduced with permission from ref. 178. Copyright 2011 the Royal Society of Chemistry. (b) EQE as a function of the brightness of all $\operatorname{Ir}(\mathrm{ppy})_{2}(\mathrm{acac})$ devices with and without MLAs. Reproduced with permission from ref. 181. 2016 Wiley-VCH Verlag GmbH \& Co. KGaA, Weinheim. (c) Schematic of molecular orientation and intermolecular interaction of $\mathrm{Ir}$ complexes on a host film under vacuum deposition. Reproduced with permission from ref. 182. Copyright 2016 American Chemical Society. (d) Measured horizontal transition dipole moment ratios of TCzTrz (data points) obtained using angle-dependent PL data and calculated by optical simulation (lines). Reproduced with permission from ref. 183. Copyright 2018 Wiley-VCH Verlag GmbH \& Co. KGaA, Weinheim.

uniform distribution of the electron density in the HOMO of the donor. As a consequence, a high EQE of over $30 \%$ was obtained without any other light-extraction method. Until now, orientation engineering of emitting dipoles has attracted more and more attention for its simple and cost-effective characteristics.

\section{Flexible and stretchable configurations}

\subsection{Flexible OLEDs}

Because of the compatibility with curvilinear and soft materials, mechanically flexible OLEDs are an emerging technology for flexible intelligent electronics with applications in bendable smartphones, curved displays, and flexible solid-state lighting devices. ${ }^{184,185}$ To fabricate high-performance flexible OLEDs, the key issue is to attain efficient flexible transparent conductive electrodes (TCEs) with excellent electrical, optical and mechanical properties. It is known that the most commonly used TCE of ITO cannot meet the requirements of flexible OLED technology due to several drawbacks including brittleness, material scarcity, and a low-throughput deposition process. ${ }^{186,187}$ Among all the alternative flexible TCEs, four types have very lately attracted great interest and shown promising progress for highly powerefficient flexible OLEDs.

5.1.1. Silver nanowires. Among all of these materials for preparing TCEs, silver nanowires hold the greatest potential in flexible electronic applications, considering they exhibit excellent optical and electronic as well as high mechanical and physical stability. ${ }^{188,189}$ Besides, TCEs based on silver nanowires can be produced through simple and cost-effective solution processes (e.g. spin-coating, spray-coating, doctor-blading, drop-casting, printing), which is compatible with popular "roll-to-roll" manufacturing. ${ }^{190-194}$ Apart from the solution process, silver-nanowire electrodes can also be constructed by the conventional thermal evaporation and post-etching method. For instance, a junction-free silver-nanowire electrode is fabricated by the wet etching of deposited metal thin films, and the electrospun polymer fibers are used as an etch mask (see Fig. 15a). ${ }^{195}$ In order to fabricate high-performance flexible OLEDs based on silver-nanowire TCEs, one critical issue that has to be settled is the high surface roughness of silver nanowire films, which may lead to severe leaking current or even short circuit of devices. For example, by embedding silver 
(a)

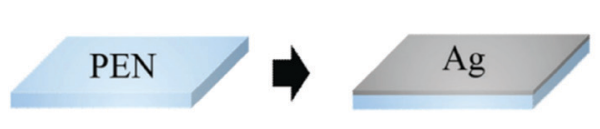

$\mathrm{Ag}$ deposition

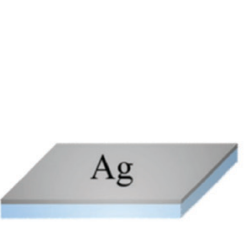

PS fiber formation

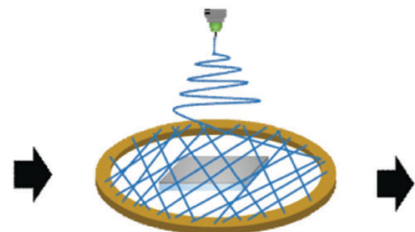

Baking at $130^{\circ} \mathrm{C}$

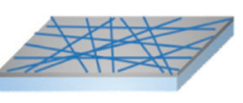

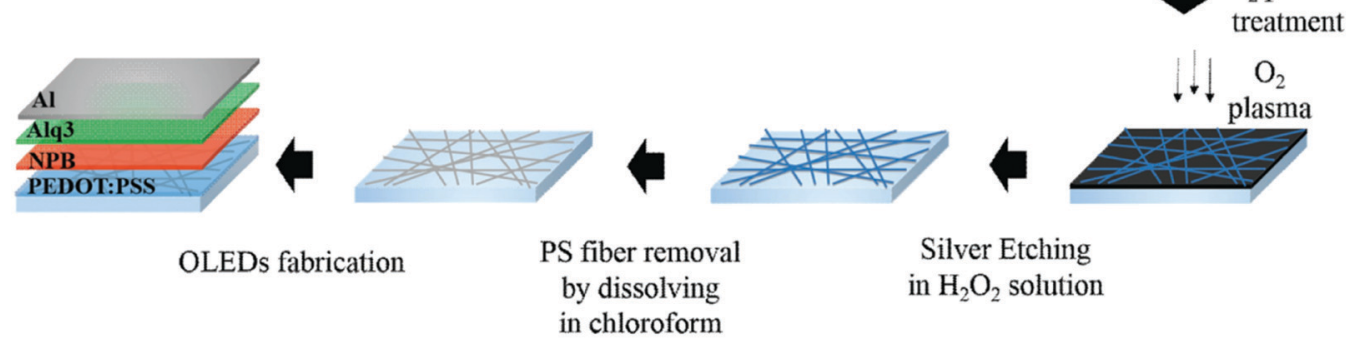

\section{(b)}
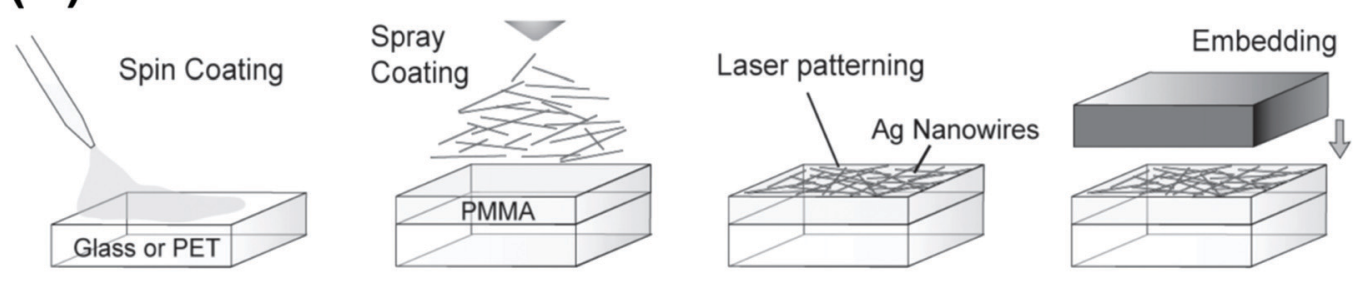

(c)

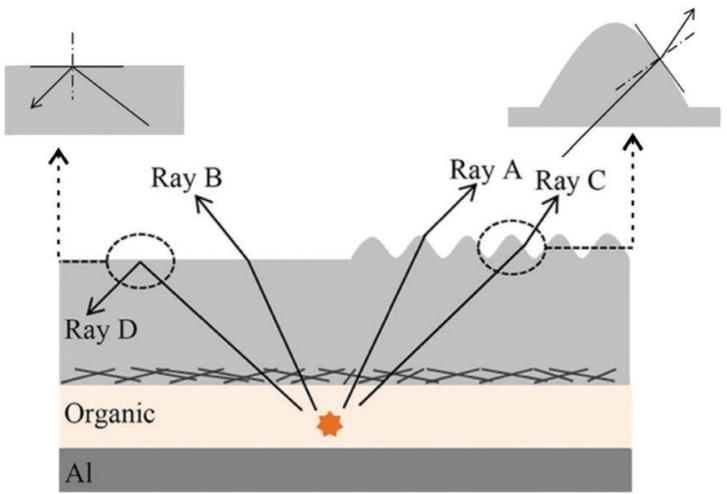

(d)

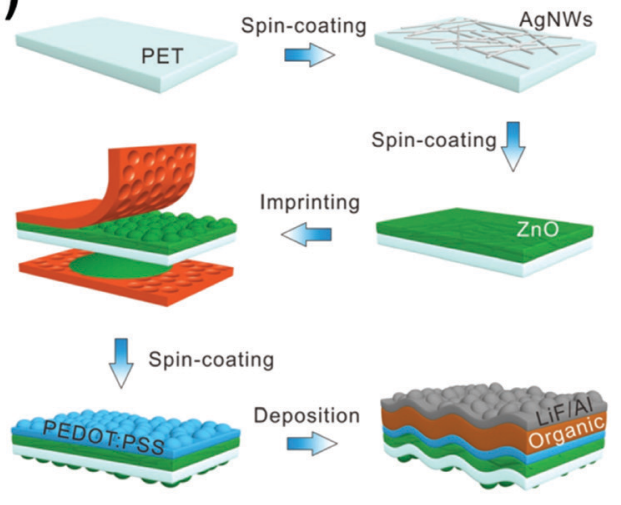

Fig. 15 (a) Schematic of the fabrication procedure of the Ag fiber electrodes and OLEDs. Reproduced with permission from ref. 195. Copyright 2017 Wiley-VCH Verlag GmbH \& Co. KGaA, Weinheim. (b) Scalable step-by-step composite electrode fabrication process. Reproduced with permission from ref. 196. Copyright 2013 Wiley-VCH Verlag GmbH \& Co. KGaA, Weinheim. (c) The schematic diagram of an OLED prepared on the nanocomposite substrate and the optical mechanism for improving the out-coupling efficiency by means of periodic wrinkles. Reproduced with permission from ref. 197. Copyright 2018 Wiley-VCH Verlag GmbH \& Co. KGaA, Weinheim. (d) Schematic illustration of the fabrication process for white flexible OLEDs, including the spin coating of AgNWs, the patterning of a sol-gel-derived ZnO layer, the spin coating of a PEDOT:PSS layer, and the subsequent deposition of organic layers. Reproduced with permission from ref. 198. Copyright 2019 Wiley-VCH Verlag GmbH \& Co. KGaA, Weinheim.

nanowires into poly(methyl methacrylate) (PMMA) films as displayed in Fig. 15b, Gaynor and co-workers successfully fabricated ITO-free white OLEDs showing a power efficiency of over $30 \mathrm{~lm} \mathrm{~W}^{-1} \cdot{ }^{196}$ During their experimental process, silver nanowires were randomly distributed by spray-coating and the embedding behavior was for reducing the surface roughness of the electrode. Particularly, to further improve the performance of OLEDs based on silver nanowires, Pei and co-workers explored efficient production schemes for flexible white OLEDs by employing silver nanowires as the conductive medium in an acrylic polymer nanocomposite substrate as shown in Fig. 15c. ${ }^{197}$ In their work, silver nanowires were embedded inside the smooth side of the substrate and external periodic wrinkles directly formed on the opposite side to enhance the outcoupling efficiency, by which the EQE of flexible white OLED reached $(38.4 \pm 2.7) \%$. Most recently, Tang and co-workers fabricated 
(a)
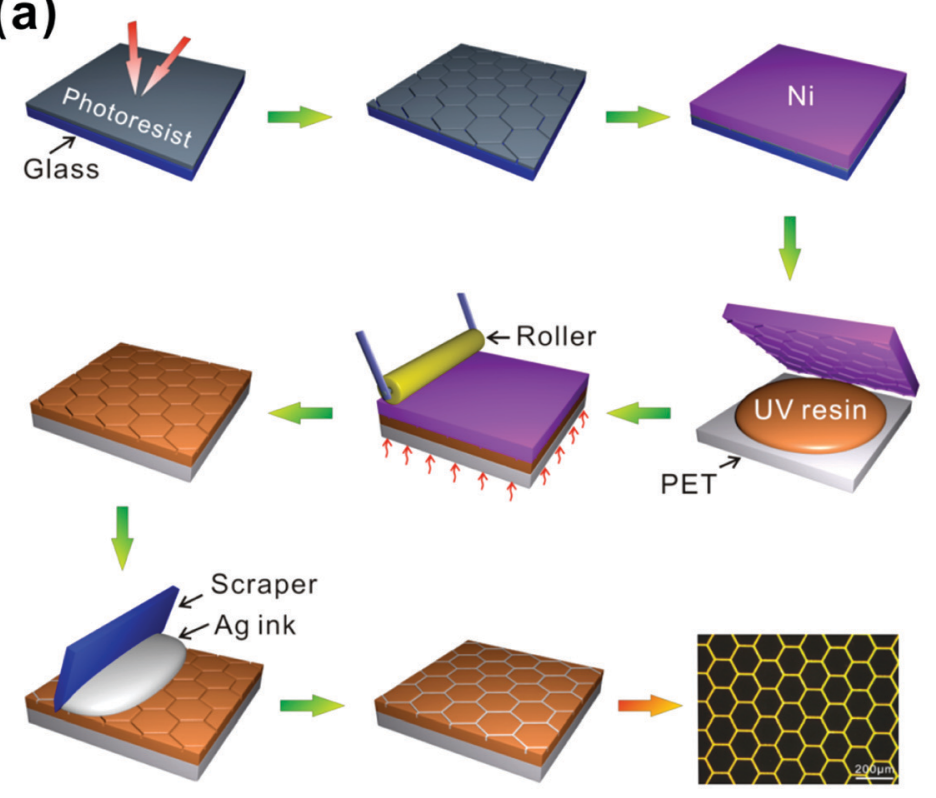

(b)
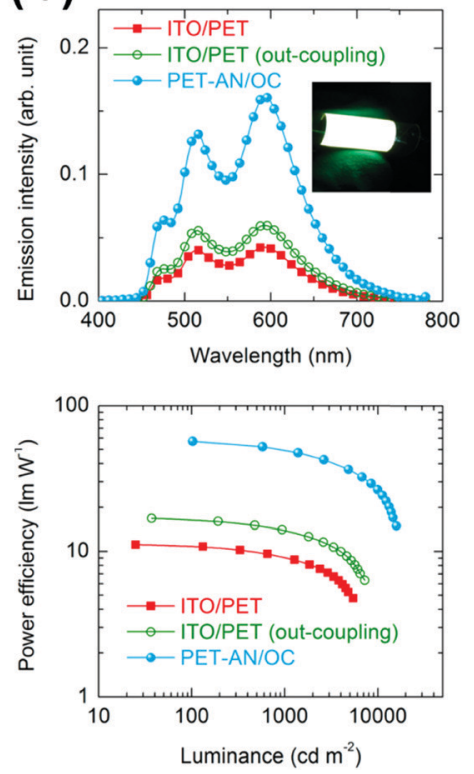

Fig. 16 (a) Schematic illustration of the fabrication steps of an embedded Ag network on a PET substrate, including photolithography, electroforming, UV nanoimprinting lithography, plasma treating, silver-paste scratching, etc. Reproduced with permission from ref. 201. Copyright 2014 American Chemical Society. (b) EL spectra of various white FOLEDs at the same current density in the normal direction and power efficiency as a function of luminance. Reproduced with permission from ref. 202. Copyright 2015 American Chemical Society.

flexible TCEs by spin-coating a silver nanowire dispersion onto a PET substrate, on which sol-gel zinc oxide was deposited for smoothing the surface of the silver nanowire TCE as demonstrated in Fig. 15d. ${ }^{198}$ By patterning nanostructures on both sides of TCE/PET intended for enhancing light outcoupling, flexible white OLEDs yield a high EQE of over $60 \%$.

5.1.2. Metal networks. Apart from metal nanowires (especially silver nanowires), metal networks (e.g. silver grid, copper mesh) have as well attracted great attention in flexible TCE applications, which are mainly prepared by the solution process, such as inkjet printing, direct ink writing, screen printing, and electroless plating. ${ }^{199,200}$ One particular advantage of using a metal network is that the line width, line spacing, and thickness of the metal mesh can be precisely controlled, hence the electrical and optical properties can be adjusted for specific requirements of flexible TCEs. However, similar to silver nanowire TCEs, flexible TCEs based on metal networks may also cause electrical leakage or short-circuits in OLEDs due to the mismatch between the thickness of embossed metal grids (several micrometers) and the extremely thin organic layer (a few hundred nanometers). The following content summarizes a typical method dealing with this issue and thus for fabricating high-performance flexible OLEDs based on silver networks. As reported by Zhou and co-workers, the electrode fabrication process can be time-saving and cost-effective by combining Ag paste scratch technology, nanoimprinting lithography, and precise pattern photolithography as displayed in Fig. $16 \mathrm{a} .{ }^{201} \mathrm{By}$ using a modified PEDOT:PSS film $(80 \mathrm{~nm})$ as the hole transporting layer, the surface roughness is reduced to $8.7 \mathrm{~nm}$. The resulting large-area $\left(12 \times 12 \mathrm{~mm}^{2}\right)$ flexible OLEDs with enhanced light outcoupling show a PCE of over $120 \mathrm{~lm} \mathrm{~W}^{-1}$ at a brightness of $1000 \mathrm{~cd} \mathrm{~m}^{-2}$ for green emission and $106 \mathrm{~lm} \mathrm{~W}^{-1}$ for white emission with an EQE of $\sim 49 \%$. Based on the same flexible silver-network TCE, they further produced efficient non-doped flexible OLEDs combined with moth-eye nanostructures for extracting light trapped in waveguide mode, substrate mode and SPP mode (Fig. 16b). ${ }^{202}$ Besides, silver network TCEs based on the above scheme show excellent bending stability, which ensures high mechanical stability for relevant flexible OLEDs.

5.1.3. Metal/dielectric composites. Though metal nanowires and networks have been widely applied in flexible OLEDs, metaldielectric composite electrodes (MDCEs) are still regarded as an effective TCE to replace ITO in terms of mechanical flexibility, electrical conductivity, optical transparency, and large-area film uniformity. ${ }^{203}$ MDCEs are not only compatible with flexible plastic substrates, but also weaken waveguide-mode light loss by replacing the high-index ITO electrode. However, the optical micro-cavity effect is inevitable when using MDCE electrodes, which is undesirable in display applications. Therefore, efficient schemes have to be proposed to solve this problem. For instance, $\mathrm{Lu}$ and co-workers demonstrated a new paradigm for the fabrication of high-efficient flexible OLEDs by collectively optimizing the multifunctional electrode stacks on polyethylene terephthalate (PET) substrates. The electrode stack is constructed with an architecture of $\mathrm{Ta}_{2} \mathrm{O}_{5} / \mathrm{Au} / \mathrm{MoO}_{3}$, where high-index $\mathrm{Ta}_{2} \mathrm{O}_{5}$ and $\mathrm{MoO}_{3}$ were used as the outcoupling layer and hole transporting layer respectively. In order to weaken the optical micro-cavity effect between the bottom and top electrodes, a thin semitransparent Au layer was deposited, which avoids the narrowing of the electroluminescence spectrum and the emergence of angledependent electroluminescence emission. Consequently, the 
(a)
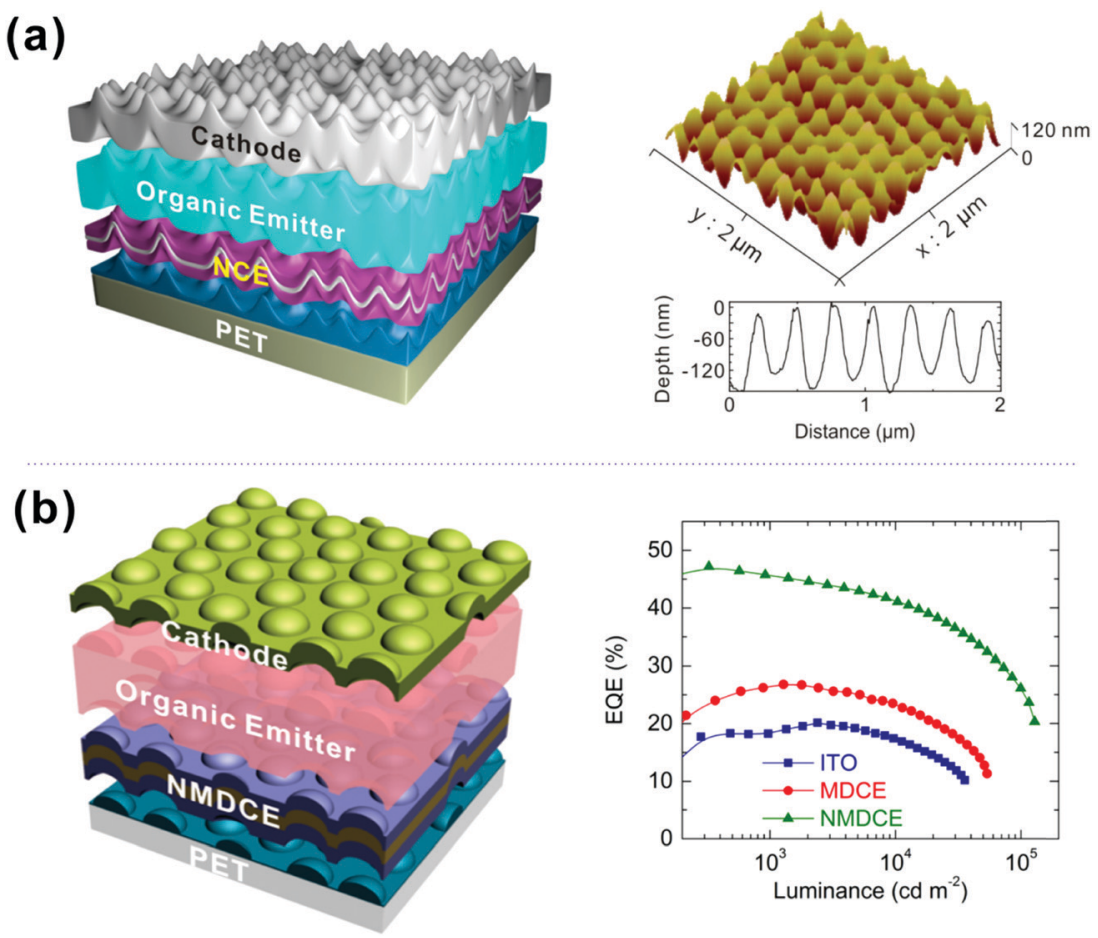

Fig. 17 (a) Schematic illustration of flexible OLEDs using a nanostructured composite electrode and the AFM profile of imprinted UV-resin layer with quasi-random nanostructures. Reproduced with permission from ref. 205. Copyright 2016 Optical Society of America. (b) Flexible OLED structure with a nanopatterned metal-dielectric electrode on a plastic substrate and EQE values as a function of luminance. Reproduced with permission from ref. 206. Copyright 2015 American Chemical Society.

maximum EQE of $63 \%$ was achieved for flexible green OLEDs with a semi-sphere lens for light out-coupling, and the EQE was as high as $60 \%$ even with a luminescence of $>10000 \mathrm{~cd} \mathrm{~m}^{-2} .^{204}$ Similarly, to alleviate the cavity issue induced by the flat metal thin film, an effective nanostructured metal/dielectric composite electrode in the form of $\mathrm{MoO}_{3} / \mathrm{Ca} / \mathrm{Ag} / \mathrm{MoO}_{3}$ was proposed for microcavity-free flexible OLEDs (Fig. 17a and b). ${ }^{205,206}$ In these devices, the quasi-random nanostructures for broadband and angle-independent light manipulation were integrated with an ultrathin Ca-doped Ag alloy film for electrical, optical and mechanical superiority. The fabricated large-area flexible white OLEDs yielded remarkably improved device performance, exhibiting a maximum power efficiency of $112.4 \mathrm{~lm} \mathrm{~W}^{-1}$.

5.1.4. Graphene. Last but not least, as an emerging type of material, the unique electrical and mechanical properties of graphene make it a promising candidate in flexible electronics. ${ }^{207,208}$ Since graphene is constructed by an atomically thin sheet of sp2hybridized carbon atoms, its inherent ultra-thin characteristic leads to high optical transmittance, a high degree of flexibility, and smooth surface topology preventing roughness-induced electrical short and degradation. Besides, its practical viability has been demonstrated. The preparation of high-quality graphene on large-scale plastic substrates has recently been developed for transparent electrodes with mass-production-compatible methods by industrial sectors. However, there are still two key shortcomings of graphene that hinder its further application in flexible OLEDs. They are the lower work-function $(-4.4 \mathrm{eV})$ and the higher sheet-resistance (usually beyond $300 \Omega \square^{-1}$ ) as compared to ITO. To date, remarkable progress on this issue has been achieved. For instance, perfluorinated ionomer (PFI) doped PEDOT:PSS is used to modify the surface of a fourlayered graphene electrode, hence providing a work-function gradient (from $4.4 \mathrm{eV}$ to $5.95 \mathrm{eV}$ ) to enhance hole injection. Moreover, the sheet-resistance of the four-layered graphene electrode is lowered to around $30 \Omega \square^{-1}$ by doping with p-dopants of $\mathrm{HNO}_{3}$ or $\mathrm{AuCl}_{3}$. Consequently, excellent flexible OLEDs are produced by modifying the graphene anode, featuring high PE $\left(37.2 \mathrm{~lm} \mathrm{~W}^{-1}\right.$ for green fluorescent OLEDs, $102.7 \mathrm{~lm} \mathrm{~W}^{-1}$ for green phosphorescent OLEDs). ${ }^{207}$ Similarly, Chen and co-workers found that the electrical properties of single-layered graphene films can be modified by doping with a small amount of triethyloxonium hexachloroantimonate (OA). The sheet-resistance is reduced from $\sim 1000 \Omega \square^{-1}$ to $<200 \Omega \square^{-1}$ as the work-function increased from $4.7 \mathrm{eV}$ to $5.1 \mathrm{eV}$ as shown in Fig. $18 \mathrm{a}^{208}$ By further employing a light outcoupling structure, flexible green OLEDs exhibit an EQE of $60 \%$, while flexible white OLEDs show EQEs reaching $45 \%$ at $10000 \mathrm{~cd} \mathrm{~m}^{-2}$ with CRI of 85. Regardless of these previous electronic and electrical modifications, the photonic role of the graphene electrodes in OLEDs has recently been investigated with a synergetic interplay of high-index $\mathrm{TiO}_{2}$ layers and low-index hole injecting layers as depicted in Fig. 18b. The sandwiched graphene electrode enabled a light out-coupling enhancement through enhancing micro-cavity resonances and suppressing SPP, which further result in a maximum EQE of $33.8 \%$ and PE of $155.8 \mathrm{~lm} \mathrm{~W}$ for flexible green OLEDs. ${ }^{209}$ 

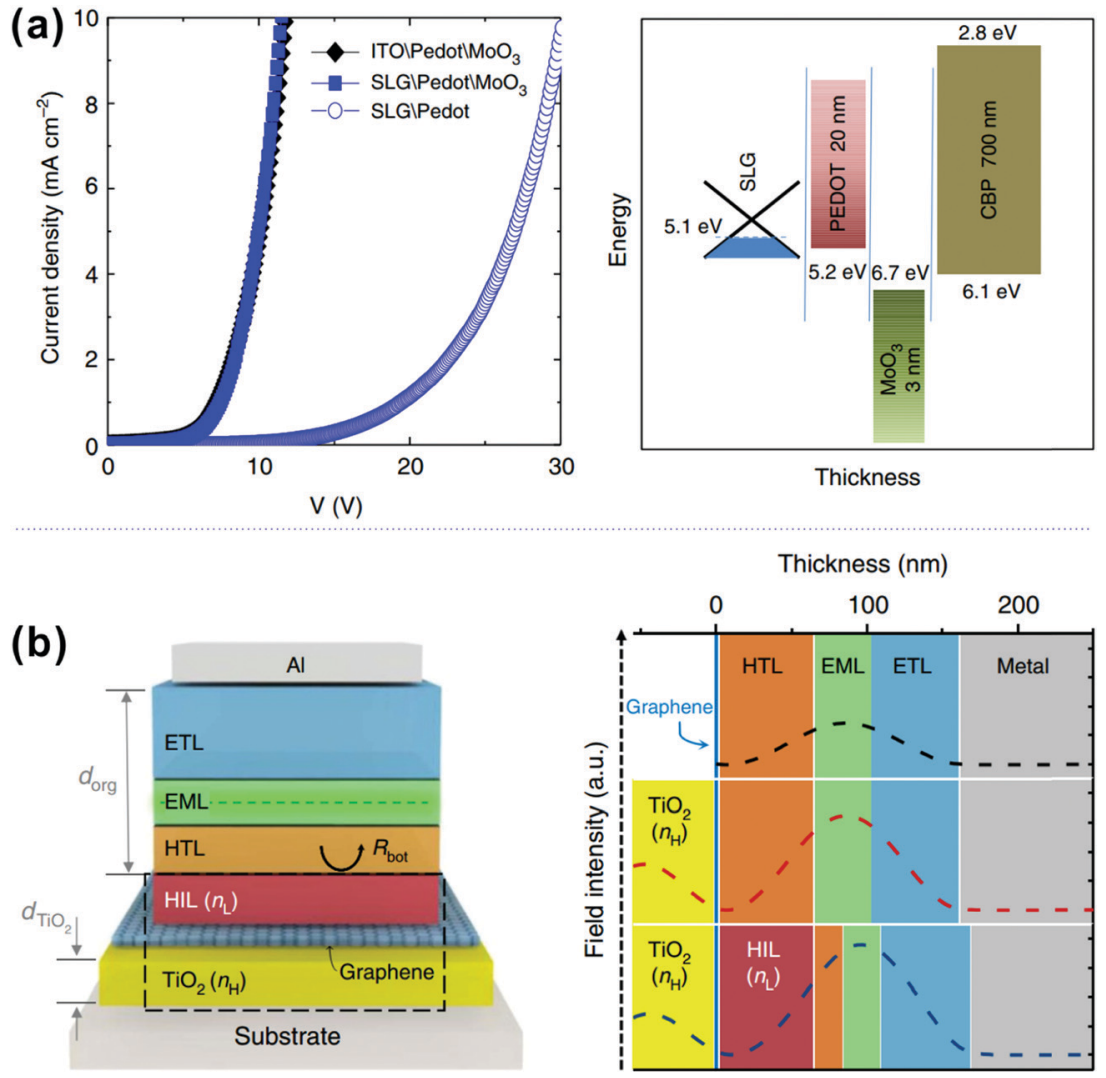

Fig. 18 (a) Energy level diagram of the single-layer graphene (SLG) anode/PEDOT:PSS/MoO $3 /$ CBP structure. Reproduced with permission from ref. 208. Copyright 2013 Macmillan Publishers Limited. (b) Schematic device structure of the proposed OLEDs and electromagnetic field intensity distribution (dashed lines) of the OLEDs under study for their respective first-order cavity design. Reproduced with permission from ref. 209. Copyright 2016 Macmillan Publishers Limited.

\subsection{Stretchable OLEDs}

Stretchable light emission devices are becoming increasingly significant in the fast-growing fields of wearable display and lighting systems, as well as biomedical and health-monitoring technologies. $^{210,211}$ The unique requirement of these devices imposed in this field is the high flexibility and stretchability, which aims at undergoing mechanical deformation at various degrees. To fabricate operational stretchable OLEDs, flexible TCEs and substrates are supposed to be prepared in advance. The intrinsically malleable polymer-based light-emitting systems are of great benefit for realizing stretchable OLED devices. For example, Sekitani and co-workers have demonstrated printable elastic conductors comprising single-walled carbon nanotubes (SWNTs) uniformly dispersed in a fluorinated rubber. ${ }^{212}$ They fabricated a rubber-like stretchable active-matrix display integrated with printed elastic conductors, organic transistors, and organic lightemitting diodes, which can be stretched by $30-50 \%$ and spread over a hemisphere without any mechanical or electrical damage. Interestingly, stretchable thin-film transistors (TFTs) have been reported by a solution printing process using a silver nanowire (AgNW)-PU acrylate (PUA) composite as the stretchable transparent electrode. Such facile TFTs were employed to drive OLEDs by controlling "on" and "off", by which stretchable lighting was realized $^{213}$ (Fig. 19a). Recently, a new efficient prototype of stretchable OLEDs has been released through a laser-programmable buckling process, showing a high efficiency of $70 \mathrm{~cd} \mathrm{~A}^{-1}$ under $70 \%$ strain and mechanical robustness with small performance fluctuations over 15000 stretch-release cycles ${ }^{214}$ (Fig. 19b). Another type of intrinsically stretchable OLED device is the utilization of polymer light-emitting electrochemical cells. In this elastomeric device, a pair of transparent composite electrodes using a thin percolation network of silver nanowires or carbon nanotubes are inlaid in the surface layer of the rubbery substrate, which allows the device to be collapsible and operable under repeated continuous stretching cycles. ${ }^{215}$ Significant progress has been made in producing fully stretchable OLEDs for practical applications in bendable and wearable electronics, especially in lighting and display applications where high luminescent performance and mechanical stability are both required. As two important roles in the architecture engineering of OLEDs, light outcoupling techniques and flexible TCEs can be synergistically employed to fabricate efficient flexible OLEDs. It is noteworthy that from the aspect of the light-extraction effect, most flexible TCEs can also be treated as an excellent light outcoupling scheme for their light-scattering or refractive-index engineering contributions.

\section{Fabrication technologies}

OLEDs can be typically fabricated in two ways, namely, the physical vapor deposition technique and solution techniques 
(a)

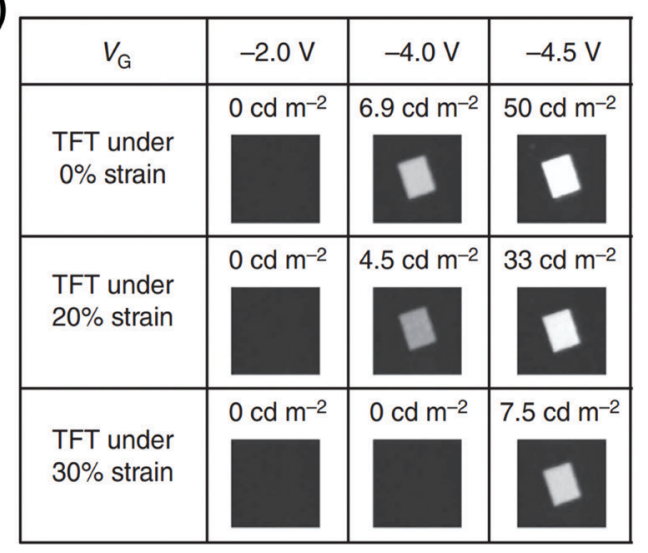

(b)

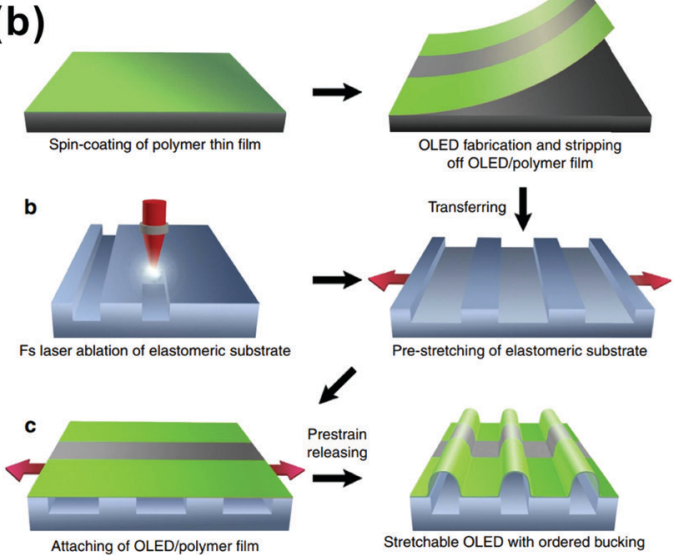

Fig. 19 (a) Luminance of an OLED driven by stretchable TFT under different inputs. Reproduced with permission from ref. 213. Copyright 2015 Macmillan Publishers Limited. (b) Schematic illustration of the fabrication process of stretchable OLEDs, including OLED preparation, substrate preparation, and stretchable OLED assembly by attaching. Reproduced with permission from ref. 214. Copyright 2016 Macmillan Publishers Limited.

including screen printing, spin-coating, inkjet printing, photolithographic process, and micro-contact printing, etc. Physical vapor deposition technology is widely used in depositing various active layers for fabricating small molecular OLEDs. This technique is applicable only for low molecular weight materials and the thermally stable lanthanide complexes. The main advantage of this technique is that it is directly amenable to complicated multilayer device structures without any serious technical problems. However, this technology is time-consuming, energy-consuming, and of limited use for large-area devices especially emerging flexible and stretchable devices. ${ }^{216}$

Compared with the vapor deposition technology, the solution process techniques are simpler and less expensive, which offer several advantages such as large-area coverage, low power consumption, and much easier device manufacture. Friend et al. developed the first polymer light-emitting diode (PLED) by a spin-coating technique using a conjugated polymer poly(phenylene-vinylene) (PPV) in 1990. ${ }^{2}$ The next year, Braun and Heeger introduced the insoluble polymer, PPV, to the soluble polymer, poly(2-methoxy-5-(2'-ethyl-hexoxy)-1,4-phenylene-vinylene) (MEH-PPV), and fabricated PLEDs by the spin-coating method. ${ }^{217}$ Hebner and co-workers fabricated OLEDs with low turn-on voltages using polyvinylcarbazole (PVK) luminescence films deposited by ink-jet printing technology. ${ }^{218}$ Müller et al. reported pixelated three-color OLED matrix displays by a spin-coating process using emitter polymers with photoresist properties. ${ }^{219}$ As depicted in Fig. 20a, Sandström et al. reported an all-solution-based flexible light-emitting electrochemical cell using a slot-die roll-coating (a)

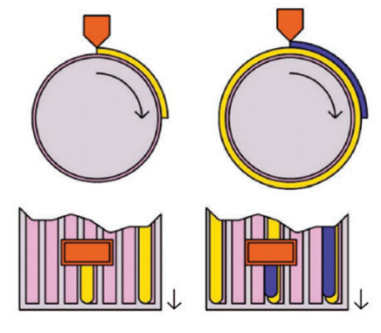

(b)

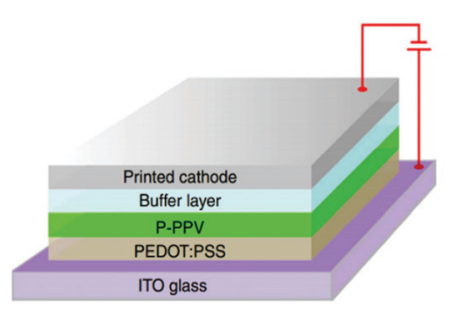

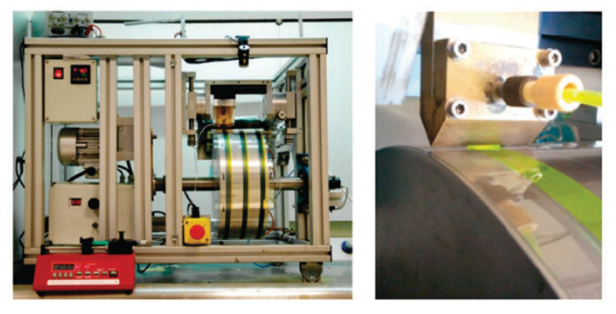

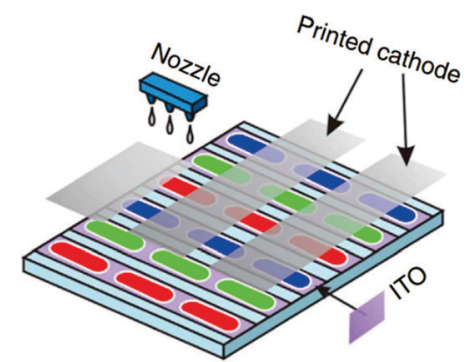

Fig. 20 (a) Schematic view of the slot-die coating of the active layer and the anode on top of the flexible cathode-coated substrate; photograph of the roll coater and close-up photograph of the slot-die head during coating of an active layer stripe. Reproduced with permission from ref. 220 . Copyright 2012 Macmillan Publishers Limited. (b) The device structure of the all-solution-processed PLED single device with the printed nanoparticle cathode and schematic illustration of the display structure of the full-color display. Reproduced with permission from ref. 216. Copyright 2013 Macmillan Publishers Limited. 
apparatus. ${ }^{220}$ The entire manufacturing procedure was carried out in air, which is directly compatible with high-speed and lowcost roll-to-roll fabrication although the constituent processed needed to be further optimized. Lately, Zheng et al. produced a 1.5-inch all-solution-processed PLED display using ink-jet conducting nanoparticles as the cathode which is deposited on a multifunctional buffer layer (Fig. 20b). ${ }^{216}$

Additionally, solution processing technology is not only used for those based on PLEDs but also extensively investigated for the fabrication of small molecule-based OLEDs (SMOLEDs). Cai et al. reported spin-coated electrophosphorescent SMOLEDs with 4,2'-bis(9-carbazolyl)-biphenyl (CBP) doped with the greenemitting tris[2-( $p$-tolyl)pyridine] iridium(III) (Ir(mppy) $\left.)_{3}\right)$, and obtained a high peak PE of $60 \mathrm{~lm} \mathrm{W^{-1 } . 2 1}$ This highperformance device implied that the spin-coating technique was an attractive and alternative route to the fabrication of multi-component devices. Zhao et al. synthesized a series of small molecular hole transport materials, which were realized by connecting the flexible moieties of butadiene bridged triphenylamine (BTPA) to $N, N, N^{\prime}, N^{\prime}$-tetraphenyl-[1,1'-biphenyl]-4,4'-diamine (TPD) (TPD(BTPA) $)_{n}(n=1,2,3,4)$. The spin-coated OLEDs with TPD(BTPA $)_{4}$ exhibited a comparable CE to devices fabricated by physical vapor deposition. ${ }^{222}$ In 2015 , Kido and co-workers reported tandem green emission OLEDs and WOLEDs comprising two phosphorescent light-emitting units using the spin-coating method. ${ }^{223}$ Here, the ZnO nanoparticle/polyethlyeneimineethoxylated (PEIE) bilayer acted as the electron injection layers and the PEDOT:PSS was adopted as the interconnecting conductive layer. The spin-coated green tandem-OLED and WOLED yielded a high CE of $94 \mathrm{~cd} \mathrm{~A}^{-1}$ and $69 \mathrm{~cd} \mathrm{~A}^{-1}$, respectively, at a current density of $5000 \mathrm{~cd} \mathrm{~m}^{-2}$.

Recently, some attractive progress has been made in transferring TADF technology to a solution processable platform, which offers a possible path to fabricating low-cost, large-area, high-performance TADF-OLEDs. In 2015, Nikolaenko et al. reported the first efficient spin-coated TADF-OLEDs with an $\mathrm{EQE}$ of $10 \%$ based on the formation of a single polymeric material containing intermonomer charge transfer state (namely intermonomer TADF) emitters. ${ }^{24}$ Chen et al. reported a nondoped and solution-processed blue OLED based on B-oTC, and obtained an EQE of $19.1 \%$ along with a CIE of $(0.15,0.26) .{ }^{225}$ Wada et al. realized a highly efficient solution-processable blue TADF-OLED with an EQE of $22.1 \%$ and CIE of $(0.15,0.19)$ by using their design strategy. ${ }^{226}$ Cho and co-workers synthesized blue TADF emitters and fabricated efficient blue TADF OLEDs using the spin-coating technique. ${ }^{227}$ This device exhibited a high EQE of $20 \%$ with color coordinates of $(0.16,0.26)$, which was comparable to that of a vapor deposited blue TADF device. Albrecht $e t$ al. reported an effective route for the synthesis of carbazole dendrons with a triphenyl-s-triazine core. ${ }^{228}$ It was the first solution-processable and non-doped high-molecular-weight TADF material. Verma et al. demonstrated TADF-OLEDs with an IQE of $13.9 \%$ by the inkjet-printing technique under atmospheric conditions based on the so-called NHetPHOS copper complex family. ${ }^{229}$ Most recently, Adachi's group reported a high efficiency, spin-coated TADF-OLED with an EQE of 9.3\% using a new class of $\pi$-conjugated polymers (namely pCzBP and pAcBP). ${ }^{230}$ In short, there are three types of emitters that can be used in solution-processable OLEDs, including small molecules, dendrimers, and polymers. Compared with the conventional thermal evaporation technique, solution-processable methods are gaining more and more attention for their low cost and simple process. It is expected that the quality of solutionprocessed OLEDs may be greatly improved through the synergistic modification of the fluid properties and printing techniques for organic solutions, which are thus probable to win a place in the future lighting and display markets.

\section{Solid-state lighting (SSL) and display technologies}

\subsection{Development and progress of WOLEDs for SSL}

Solid-state lighting (SSL), defined as the use of semiconductor electroluminescence to produce visible light for illumination, will make greener buildings that use less electricity, making life less dependent on fossil fuels. In the coming decade, SSL will become key to constructing low-cost and high-performance buildings that consume less energy and produce fewer greenhouse gas emissions than their counterparts. SSL sources based on WOLEDs have gained considerable attention of academic and industrial research communities as the next-generation lighting sources since the first report of a WOLED by Kido in 1994. ${ }^{231}$ Compared to existing light sources, such as incandescent light bulbs, fluorescent lamps, and inorganic LEDs, WOLEDs offer extreme light-weight, flexibility, light uniformity, glare-free display and thin profiles that are more comfortable for human perception. ${ }^{232-235}$ There are several key performance factors of WOLEDs for SSL, including efficiency, lifetime, and color quality. In the previous sections, we have discussed some strategies for obtaining OLEDs with high efficiency and long lifetime. As for color quality, two critical sub-factors are required for achieving a high-quality WOLED, including a limited CIE chromaticity coordinate close to the black-body curve with a correlated color temperature (CCT) between $2500 \mathrm{~K}$ and $6500 \mathrm{~K}$, and a CRI above $80 .^{3}$ Over the past two decades, great advances of WOLEDs in lighting technology have been made.

7.1.1. Evolution of WOLEDs for SSL. In academic research, the PE of WOLEDs has been improved from $0.83 \mathrm{~lm} \mathrm{~W}^{-1}$ to $>160 \mathrm{~lm} \mathrm{~W}{ }^{-1}$ over the past decades. ${ }^{236-242}$ In 2002, Adamovich et al. fabricated WOLEDs with doped CBP and MCP luminescent layers. ${ }^{236}$ The CBP- and mCP-based devices gave a PE of $7.3 \mathrm{~lm} \mathrm{~W}^{-1}$ (@ $1 \mathrm{~cd} \mathrm{~m}^{-2}$ ) and $12.2 \mathrm{~lm} \mathrm{~W}^{-1}$ (@ $17 \mathrm{~cd} \mathrm{~A}^{-1}$ ), respectively. In 2004, D'Andrade et al. reported a phosphorescent WOLED with a maximum PE of $42 \mathrm{~lm} \mathrm{~W}^{-1}$ by using a thin exciton confinement light-emitting layer. ${ }^{237}$ In 2007, a WOLED with a PE of $57.6 \mathrm{~lm} \mathrm{~W}^{-1}$ at a brightness of $100 \mathrm{~cd} \mathrm{~m}^{-2}$ was achieved by designing an appropriate structure with high exciton utilization. ${ }^{238}$ In 2009, Leo and co-workers reported a high-efficiency WOLED with a PE of $90 \mathrm{~lm} \mathrm{~W}^{-1}$ (@1000 $\mathrm{cd} \mathrm{m}^{-2}$ ). ${ }^{239}$ This value has been successfully increased to $124 \mathrm{~lm} \mathrm{~W}^{-1}$ by combining half-sphere and combining high refractive index 
glass and half-sphere to enhance the light-outcoupling. In 2014, a two-unit white OLED showed a PE of $125 \mathrm{~lm} \mathrm{~W}^{-1}$ at $100 \mathrm{~cd} \mathrm{~m}^{-2}$ and a long lifetime of $>100000 \mathrm{~h}$ at $1000 \mathrm{~cd} \mathrm{~m}^{-2}$ with the utilization of a high-refractive-index microlens array. ${ }^{240}$ In addition, a white Ph-OLED with a PE of $105 \mathrm{~lm} \mathrm{~W}^{-1}$ without light extraction structure was reported by Fung et al. in $2017 .^{117}$ In 2018, Kim et al. reported a high-efficiency WOLED with an excellent EQE of $78 \%$ and a PE of $164 \mathrm{~lm} \mathrm{~W}^{-1}$ by employing both internal (random vacuum nanohole array) and external light extraction structures (half-spherical lens). ${ }^{241}$ In the same year, Tang et al. reported a transparent flexible WOLED with a nanopatterned metal-dielectric composite electrode, and the WOLED exhibited an excellent EQE of $72.4 \%$ and an extremely high PE of $168.5 \mathrm{~lm} \mathrm{~W}^{-1}$ with a CRI of above $84 .^{242}$

In the industry field, considerable efforts have been devoted so far to realizing high-performance WOLEDs for SSL applications. In 2005, GE Global Research fabricated a $2 \mathrm{ft} \times 2 \mathrm{ft}$ white OLED panel with a PE of $15 \mathrm{~lm} \mathrm{~W}^{-1}$, a CCT of $4400 \mathrm{~K}$ and a CRI of 88 (Fig. 21a). ${ }^{243}$ In 2008, Research Institute of Organic Electronics in Japan demonstrated $14 \mathrm{~cm} \times 14 \mathrm{~cm}$ prototyping WOLED panels with a PE of $20 \mathrm{~lm} \mathrm{~W}^{-1}$ (@5000 $\mathrm{cd} \mathrm{m}^{-2}$ ). ${ }^{244}$ In 2010, Panasonic company reported high-quality WOLED panels with PE $>30 \mathrm{~lm} \mathrm{~W}{ }^{-1}$, CRI $>90$ and half-decay lifetime $>40000 \mathrm{~h}$ (@1000 cd $\mathrm{m}^{-2}$ ). Moreover, the lifetime was improved to $80000 \mathrm{~h}$ by using an efficient heat radiation thin encapsulation structure. ${ }^{245}$ In the same year, Universal Display Corporation developed two $15 \mathrm{~cm} \times 15 \mathrm{~cm}$ OLED lighting panels: Panel 1 and Panel 2. Panel 1 exhibited a PE of $58 \mathrm{~lm} \mathrm{~W}{ }^{-1}$, CRI of 86 and CCT of 2790 (see Fig. 21b), while Panel 2 showed a PE of $50 \mathrm{~lm} \mathrm{~W}^{-1}$, CRI of 87, CCT of 3055 and LT70 of 10000 h. $^{246}$ In 2018, OLED Works company used thin glass substrates to fabricate a bendable OLED lighting panel, and the bending radius of the panel can be decreased to $10 \mathrm{~cm}$ as shown in Fig. 21c. ${ }^{247}$ In addition to the plane lighting panel, the kirigami-based 3D OLED lighting has also been reported by Kim et al. in $2018 .^{248}$

(a)

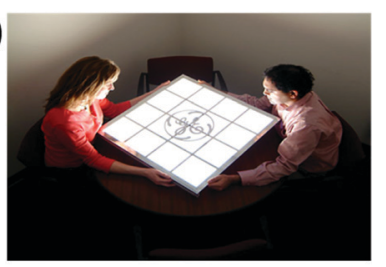

(c)

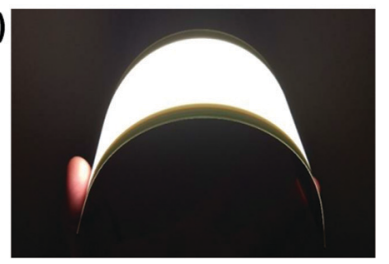

Fig. 21 Commerical and potential applications of WOLEDs for solid-state lighting. $(\mathrm{a}-\mathrm{c})$ WOLED lighting panels developed by commercial companies. Reproduced with permission from ref. 243, 246 and 247. Copyright 2005-2018 SID. (d) OLED tulip illumination. Reproduced with permission from ref. 249. Copyright 2017 John Wiley \& Sons, Inc.
OLED lighting panels have many advantages, such as extreme lightweight, thin profile, glare-free display, flexibility and light uniformity, allowing various attractive designs and innovative applications. For example, beautiful tulip lighting flowers can be fabricated by using flexible plane OLEDs as light sources, while conventional point, linear and circular light sources could not make it possible (Fig. 21d). ${ }^{249}$ In addition, Philips fabricated an interactive OLED mirror, which can be applied in the hallway or next to the cloakroom. ${ }^{250}$ Moreover, WOLEDs can be used as portable lighting, which is ideal for use during plant 'shutdowns' for maintenance and installation requirements. Alkilu developed a portable OLED light panel, which is about $10 \times 10 \mathrm{~cm}$ in size and has a color temperature of $3000 \mathrm{~K}$. More importantly, this apparatus has a Li-polymer battery that can last for more than 30 hours. ${ }^{251}$ Japan-based Stanley Electric developed an OLED tail light prototype and aimed to commercialize such a tail lamp by $2020 .{ }^{252}$ Therefore, it is doubtless that high-performance novelty OLED products with low material loss and higher added values will boost the potential lucrative niche markets of SSL.

7.1.2. Physiological-friendly light sources. Nowadays, although various artificial lighting applications are commercialized, most of such equipment is not human-friendly because of strong radiation with intense energy, which may potentially influence macular health and human hormones. ${ }^{253,254}$ In contrast, the emitting spectrum of WOLEDs can be easily modified, which provides tantalizing advantages for creating physiological-friendly light sources (e.g., sunlight, candlelight). To have sunlight-style illumination, emission with daylight chromaticity and a wide color-temperature span is essential. In 2009, Jou and co-workers reported a sunlight-style WOLED with a tunable color temperature between 2300 and $8200 \mathrm{~K}$ with a power efficiency of $7.0 \mathrm{~lm} \mathrm{~W}^{-1}$ and $2.2 \mathrm{~lm} \mathrm{~W}^{-1}$ at a brightness of $100 \mathrm{~cd} \mathrm{~m}^{-2}$ and $1000 \mathrm{~cd} \mathrm{~m}^{-2}$, respectively ${ }^{255}$ (Fig. 22a). Such a device can reflect diverse sky situations at various driving voltages. Later, Yu et al. developed color-tunable sunlight-like WOLEDs with a high CRI of 89 at $5000 \mathrm{~cd} \mathrm{~m}^{-2} \cdot{ }^{256}$ This wide color-temperature span of the four-peak device could meet requirements for lighting in dwellings. Recently, Wu et al. proposed dimmable sunlight-like WOLEDs that were fabricated using three luminophores to form an emitting spectrum similar to black body radiation at $2250 \mathrm{~K}$ with an ultra-high CRI value of 91 (Fig. 22b). ${ }^{257}$ Meanwhile, to respond to the call for a human-friendly light at night that shows low color temperature, researchers also paid attention to developing candle light-style OLEDs. In 2011, Jou and co-workers demonstrated the incorporation of a blend interlayer between emissive layers to improve the device performance of low color temperature OLEDs. ${ }^{258}$ This optimal device with $30 \mathrm{~lm} \mathrm{~W}^{-1}$ at $1000 \mathrm{~cd} \mathrm{~m}^{-2}$ exhibits much lower color temperature than that of incandescent bulbs, and as low as that of candles. Later, Jou et al. presented another candle light-style OLED composed of four organic electro-phosphorescence dyes with red, yellow, green and sky-blue colors. ${ }^{259}$ This device can create an emission spectrum mimicking that of candlelight with a color temperature as low as $1900 \mathrm{~K}$, CRI as high as 93, and efficiency at least two times that of incandescent bulbs (Fig. 22c). A red wine 


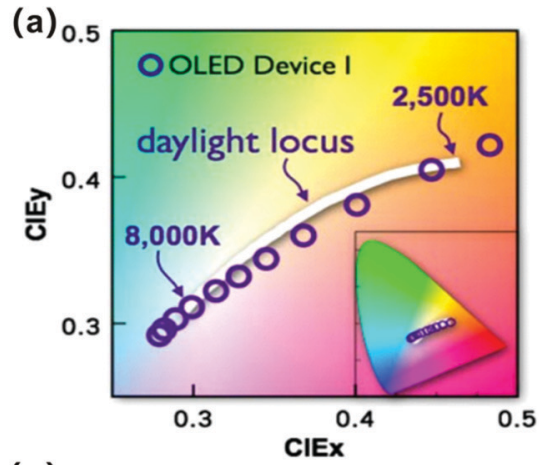

(c)

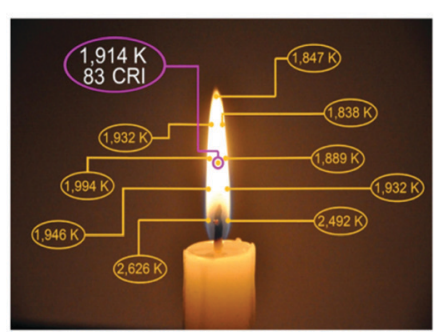

(b)

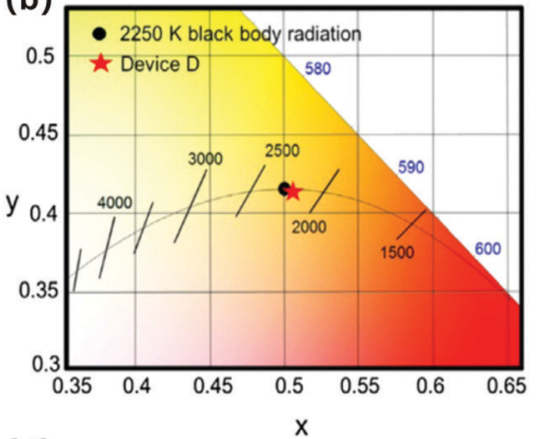

(d)

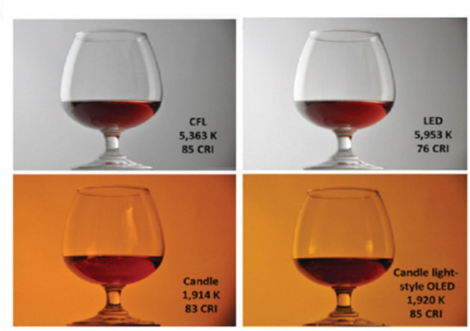

Fig. 22 Optimized physiologically friendly light sources. (a) Chromaticity and color-temperature characteristics of the sunlight-style OLED. Reproduced with permission from ref. 255. Copyright 2009 American Institute of Physics. (b) ClE chromaticity coordinates of WOLEDs with the solar spectrum. Reproduced with permission from ref. 257. Copyright 2016 Elsevier. (c) Candle shows different color temperatures at different flame positions. Reproduced with permission. Reproduced with permission from ref. 259. Copyright 2013 Wiley-VCH Verlag GmbH \& Co. KGaA, Weinheim. (d) Lighting color temperature effect on the photographic results of a wine containing glass illuminated by different light sources. Reproduced with permission. Reproduced with permission from ref. 259. Copyright 2013 Wiley-VCH Verlag GmbH \& Co. KGaA, Weinheim.

containing glass displayed similar appearance while illuminated with white candle and candle light-style OLEDs (Fig. 22d). Thereby, together with the characteristic of modified spectrum of OLEDs, these make them ideal future lighting sources for ultimate illumination quality.

\subsection{Development and progress of OLED displays}

OLED displays have appeared on the flat-panel display market to compete against conventional cathode-ray tubes (CRTs) and liquid-crystal displays (LCDs) since the mid-1980s. ${ }^{260,261}$ The main feature is that OLED displays can perfectly control the variations of brightness and generate color within subpixels of red, green, and blue colors inside a panel, whereas LCD displays are transmissive devices which can't fully block the backlight. Compared with an LCD display, an OLED display has a thinner body, higher color contrast, faster response speed, potentially lower power consumption and even lower cost since the latter does not require a backlight. ${ }^{262}$ And the OLED can achieve a flexible display, which is difficult for the LCD. However, there are still some urgent problems to be solved in OLEDs. For example, the relatively faster decay of commercial blue emitters than green and red emitters during the operation led to a color deviation, especially in large size display screens. Although some strategies were proposed to compensate for the deficiency of blue emission, developing commercial blue emitters with high efficiency and good stability is still necessary. ${ }^{263,264}$ For now, at least in small-size display screens, such as smartwatches and smartphones, OLEDs have obvious advantages over LCDs.
Nowadays, OLED displays have already been extensively used in Audio/Video players, digital cameras, wearable devices, mobile phones, laptops, and OLED TV displays. For example, in 2010, Sony Corporation fabricated an Audio player with a 2.8-inch OLED display and a resolution of $240 \times 240$ pixels. ${ }^{265}$ In 2016, Sony also introduced a new type of digital camera with $3 \mathrm{~K}$ OLED viewfinder. ${ }^{266}$ At the same time, various digital camera manufacturers (e.g., Panasonic, Fujifilm, Nikon) have introduced new high-quality products with OLED displays. In addition, the worldwide wearable device market will continue to grow in the future. Various stylish wearable devices have begun to adopt OLED displays for superior color quality, such as fitness bands and head-mounted displays. For instance, the Apple Watch Series 2 as the 2nd-generation smartwatch has adopted an OLED display with $272 \times 340$ pixels. In particular, the Apple Watch Series 5 as all-weather retina OLED display adopted low-temperature polycrystalline oxide (LTPO) backplane technology. ${ }^{267}$ Simultaneously, OLED displays can be used in virtual reality (VR) displays for 3D viewing because of the high resolution and high degree of comfort. The OLED technology is also used in many smartphones today, for instance, the iPhone X has adopted a $5.8^{\prime \prime} 1125 \times 2436$ AMOLED display. ${ }^{268}$ For OLED TVs, since 2010, LG developed a 15-inch $1366 \times 768$ HD TV and has been constantly developing large-size high resolution OLED TVs that can compete with high-end LED/ LCD TVs (Fig. 23). ${ }^{263,269-273}$ By 2015 LG developed 55", 65", 77" flexible $4 \mathrm{~K}$ ultra high definition (UHD) TVs. ${ }^{271-273}$ In 2016, LG display demonstrated a $55^{\prime \prime}$ full high definition (FHD) transparent OLED TV. ${ }^{263}$ Moreover, OLED display technology is also 
(a)

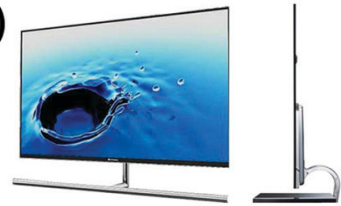

55” OLED TV, 2012

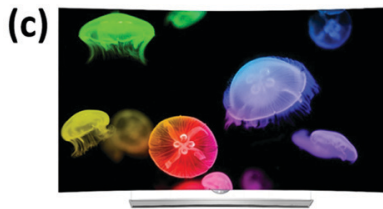

65" UHD OLED TV, 2015 (b)

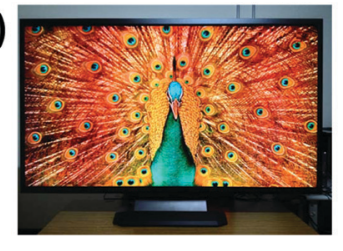

65" OLED TV, 2013

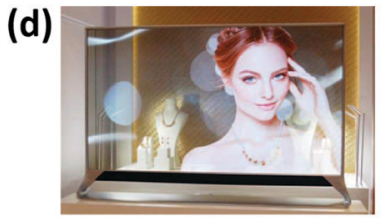

55" FHD transparent OLED TV, 2016
Fig. 23 The OLED TVs developed by LG displays. Reproduced with permission from ref. 263, 269, 270 and 272. Copyright 2012-2017 SID.

widely adopted in computer products (e.g., Dell Computer Corporation, Hewlett-Packard Development Company), digital recording binoculars, and portable video magnifiers. Alienware developed and commercialized the latest gaming laptop with 13-inch $2560 \times 1440$ OLED displays. ${ }^{274}$ This laptop is similar to the previous product (LCD Alienware 13 R2), just with an OLED instead of the LCD display. Similarly, in some other commercial products, OLED displays have gradually been adopted. For instance, Sony introduced a binocular-camera that stands alone in the digital binocular market - it records $2 \mathrm{D}$ and $3 \mathrm{D}$ video in full high definition and features a 5 -inch touch OLED display. ${ }^{275}$ If this trend continues, we might conclude that the OLED display technology would become mainstream in a couple of years.

\section{Summary and perspectives}

In this paper, we have systematically reviewed recent advances in OLED materials, device architectures, light extraction approaches, fabrication techniques, as well as breakthroughs in organic SSL and displays. Different materials and the corresponding emission principle have been firstly summarized. Some advanced mechanisms, such as TADF and TTA, might unlock the theoretical limit of OLED devices. A variety of light extraction schemes with the implementation of micro-nano structures have been demonstrated to contribute to release the trapped photons of the devices. The rapid development techniques of flexible and stretchable OLEDs provide attractive prospects for future flexible or foldable OLED-based applications. Meanwhile, solution-processed fabrication approaches, such as spin-coating and ink-jet printing, could efficiently reduce the manufacturing cost. These scientific efforts have improved the performance of OLEDs and accelerated their commercial application.

However, it is noteworthy that major technological challenges need to be overcome for OLEDs to become a success in the future. First, affordability might be a critical challenging goal, although OLEDs have already been commercialized as organic
SSL and displays in numerous consumer electronics. The manufacturing costs have to be reduced significantly so that they can compete with the existing technologies and gradually dominate the display and SSL market. An alternative way to reduce the cost is through the roll-to-roll (reel-to-reel) fabrication technologies with high throughput.

Second, the significant progress in inorganic light-emitting diodes (LEDs) as backlight sources of liquid crystal displays (LCDs) with a dramatic decrease in power consumption is weakening the competitiveness of OLEDs over LCDs. Although advanced optical light extraction engineering has made attractive progress, an important challenge for these techniques is to directly transfer the structures over a large area at a very low cost.

Thirdly, recently emerged colloidal quantum dot LEDs (QLEDs) have attracted great interest both in scientific and industry domains. QLEDs generally exhibit superior color purity compared with OLEDs, although QLED technologies are not yet mature enough to be used in SSL and displays. However, combined with blue LED-based backlight solutions, QLEDs have been successfully incorporated into today's advanced LCD displays, which might bring unexpected challenges for OLEDs' future applications.

Last, one of the disadvantages of OLEDs is their individual lifetime. The lifetime of blue devices is relatively shorter than other colored devices. The reported T95 of the red and green devices has already exceeded 10000 hours at $1000 \mathrm{~cd} \mathrm{~m}^{-2} \cdot{ }^{69}$ TADF offers many possibilities for stable blue OLEDs, but this technology is not mature enough to provide answers yet. It remains an urgent task to prevent aging of the devices.

Although the challenges facing OLEDs are significant, rapid progress in the development of OLEDs in recent years provides significant confidence in this promising technology for future lighting and displays. We believe that scientific research in OLEDs is vibrant and expanding, and OLEDs will enter our everyday life with an exciting new form-factor in the near future.

\section{Conflicts of interest}

There are no conflicts to declare.

\section{Acknowledgements}

This work was financially supported by the National Natural Science Foundation of China (No. 61520106012, 61905171, 61722404, 51873138, 91633301), the National Key R\&D Program of China (No. 2016YFB0401002, 2016YFB0400700), the Natural Science Foundation of Jiangsu Province of China (BK20190815), the 333 Program (No. BRA2019061), and the Collaborative Innovation Center of Suzhou Nano Science \& Technology.

\section{References}

1 C. W. Tang and S. A. VanSlyke, Appl. Phys. Lett., 1987, 51, 913.

2 J. H. Burroughes, D. D. C. Bradley, A. R. Brown, R. N. Marks, K. Mackay, R. H. Friend, P. L. Burns and A. B. Holmes, Nature, 1990, 347, 539. 
3 B. W. D'Andrade and S. R. Forrest, Adv. Mater., 2004, 16, 1585.

4 G. Schwartz, S. Reineke, T. C. Rosenow, K. Walzer and K. Leo, Adv. Funct. Mater., 2009, 19, 1319.

5 J. Lee, H. F. Chen, T. Batagoda, C. Coburn, P. I. Djurovich, M. E. Thompson and S. R. Forrest, Nat. Mater., 2015, 15, 92.

6 C. Y. Kuei, W. L. Tsai, B. Tong, M. Jiao, W. K. Lee, Y. Chi and P. T. Chou, Adv. Mater., 2016, 28, 2795.

7 Y. J. Cho, K. S. Yook and J. Y. Lee, Adv. Mater., 2014, 26, 4050 .

8 M. Kim, S. K. Jeon, S. H. Hwang and J. Y. Lee, Adv. Mater., 2015, 27, 2515.

9 L. S. Hung and C. H. Chen, Mater. Sci. Eng., R, 2002, 39, 143.

10 C. Lee and J. J. Kim, Small, 2013, 9, 3858.

11 T. Yamasaki, K. Sumioka and T. Tsutsui, Appl. Phys. Lett., 2000, 76, 1243.

12 K. Meerholz and D. C. Müller, Adv. Funct. Mater., 2001, 11, 251.

13 D. Zhang, K. Ryu, X. Liu, E. Polikarpov, J. Ly, M. E. Tompson and C. Zhou, Nano Lett., 2006, 6, 1880.

14 D. S. Hecht, L. Hu and G. Irvin, Adv. Mater., 2011, 23, 1482.

15 M. Pope and C. E. Swenberg, Electronic processes in organic crystals and polymers, Oxford University Press on Demand, 1999.

16 M. A. Baldo, D. F. O'brien and S. R. Forrest, Phys. Rev. B: Condens. Matter Mater. Phys., 1999, 60, 14422.

17 T. Arakane, M. Funahashi, H. Kuma, K. Fukuoka, K. Ikeda, H. Yamamoto, F. Moriwaki and C. Hosokawa, SID Symp. Dig. Tech. Pap., 2006, 37, 37.

18 C.-J. Chiang, A. Kimyonok, M. K. Etherington, G. C. Griffiths, V. Jankus, F. Turksoy and A. P. Monkman, Adv. Funct. Mater., 2013, 23, 739.

19 D. Y. Kondakov, Philos. Trans. R. Soc., A, 2015, 373, 2014032.

20 Y. Luo and H. Aziz, Adv. Funct. Mater., 2010, 20, 1285.

21 J. Zhou, P. Chen, X. Wang, Y. Wang, Y. Wang, F. Li, M. Yang, Y. Huang, J. Yu and Z. Lu, Chem. Commun., 2014, 50, 7586.

22 X. Tang, Q. Bai, T. Shan, J. Li, Y. Gao, F. Liu, H. Liu, Q. Peng, B. Yang, F. Li and P. Lu, Adv. Funct. Mater., 2018, 28, 1705813.

23 M. H. Hsin, C. Y. Lee, Y. C. Chen, P. Y. Chen, Y. H. Chen, H. H. Lu, Y. H. Lin, B. Y. Lin, M. Z. Lee, T. L. Chiu, C. F. Lin and J. H. Lee, SID Symp. Dig. Tech. Pap., 2016, 47, 1727.

24 B. Y. Lin, M. Z. Lee, P. C. Tseng, J. H. Lee, T. L. Chiu, C. F. Lin, J. Hsieh and S. Chen, SID Symp. Dig. Tech. Pap., 2017, 48, 1928.

25 B. Y. Lin, C. J. Easley, C. H. Chen, P. C. Tseng, M. Z. Lee, P. H. Sher, J. K. Wang, T. L. Chiu, C. F. Lin, C. J. Bardeen and J. H. Lee, ACS Appl. Mater. Interfaces, 2017, 9, 10963.

26 C. H. Chen, N. T. Tierce, M. K. Leung, T. L. Chiu, C. F. Lin, C. J. Bardeen and J. H. Lee, Adv. Mater., 2018, 30, e1804850.

27 D. Hu, L. Yao, B. Yang and Y. Ma, Philos. Trans. R. Soc., A, 2015, 373, 20140318.

28 W. Li, Y. Pan, R. Xiao, Q. Peng, S. Zhang, D. Ma, F. Li, F. Shen, Y. Wang, B. Yang and Y. Ma, Adv. Funct. Mater., 2014, 24, 1609.
29 W. Li, D. Liu, F. Shen, D. Ma, Z. Wang, T. Feng, Y. Xu, B. Yang and Y. Ma, Adv. Funct. Mater., 2012, 22, 2797.

30 L. Yao, S. Zhang, R. Wang, W. Li, F. Shen, B. Yang and Y. Ma, Angew. Chem., Int. Ed., 2014, 53, 2119.

31 X. Tang, Q. Bai, Q. Peng, Y. Gao, J. Li, Y. Liu, L. Yao, P. Lu, B. Yang and Y. Ma, Chem. Mater., 2015, 27, 7050.

32 B. Liu, Z.-W. Yu, D. He, Z.-L. Zhu, J. Zheng, Y.-D. Yu, W.-F. Xie, Q.-X. Tong and C.-S. Lee, J. Mater. Chem. C, 2017, 5, 5402 .

33 H. Zhang, J. Zeng, W. Luo, H. Wu, C. Zeng, K. Zhang, W. Feng, Z. Wang, Z. Zhao and B. Z. Tang, J. Mater. Chem. C, 2019, 7, 6359.

34 M. Kasha, Discuss. Faraday Soc., 1950, 9, 14, DOI: 10.1039/ DF9500900014.

35 H. Uoyama, K. Goushi, K. Shizu, H. Nomura and C. Adachi, Nature, 2012, 492, 234.

36 S. Y. Lee, T. Yasuda, Y. S. Yang, Q. Zhang and C. Adachi, Angew. Chem., Int. Ed., 2014, 53, 6402.

37 Y. Shu and B. G. Levine, J. Chem. Phys., 2015, 142, 104104.

38 D. H. Ahn, S. W. Kim, H. Lee, I. J. Ko, D. Karthik, J. Y. Lee and J. H. Kwon, Nat. Photonics, 2019, 13, 540.

39 T.-L. Wu, M.-J. Huang, C.-C. Lin, P.-Y. Huang, T.-Y. Chou, R.-W. Chen-Cheng, H.-W. Lin, R.-S. Liu and C.-H. Cheng, Nat. Photonics, 2018, 12, 235.

40 Y. L. Zhang, Q. Ran, Q. Wang, Y. Liu, C. Hanisch, S. Reineke, J. Fan and L. S. Liao, Adv. Mater., 2019, 31, e1902368.

41 A. Endo, K. Sato, K. Yoshimura, T. Kai, A. Kawada, H. Miyazaki and C. Adachi, Appl. Phys. Lett., 2011, 98, 083302.

42 M. Kim, S. K. Jeon, S. H. Hwang and J. Y. Lee, Adv. Mater., 2015, 27, 2515.

43 D. Zhang, M. Cai, Y. Zhang, D. Zhang and L. Duan, Mater. Horiz., 2016, 3, 145.

44 L. S. Cui, S. B. Ruan, F. Bencheikh, R. Nagata, L. Zhang, K. Inada, H. Nakanotani, L. S. Liao and C. Adachi, Nat. Commun., 2017, 8, 2250.

45 C. Y. Chan, M. Tanaka, H. Nakanotani and C. Adachi, Nat. Commun., 2018, 9, 5036.

46 Q. Peng, A. Obolda, M. Zhang and F. Li, Angew. Chem., Int. Ed., 2015, 54, 7091.

47 X. Ai, E. W. Evans, S. Dong, A. J. Gillett, H. Guo, Y. Chen, T. J. H. Hele, R. H. Friend and F. Li, Nature, 2018, 563, 536.

48 N. J. Turro, Modern Molecular Photochemistry, University science books, 1991.

49 V. Gamero, D. Velasco, S. Latorre, F. López-Calahorra, E. Brillas and L. Juliá, Tetrahedron Lett., 2006, 47, 2305.

50 A. Heckmann, S. Dümmler, J. Pauli, M. Margraf, J. Köhler, D. Stich, C. Lambert, I. Fischer and U. Resch-Genger, J. Phys. Chem. C, 2009, 113, 20958.

51 Y. Hattori, T. Kusamoto and H. Nishihara, Angew. Chem., Int. Ed., 2014, 53, 11845.

52 Y. Ma, H. Zhang, J. Shen and C. Che, Synth. Met., 1998, 94, 245.

53 M. A. Baldo, D. F. O’brien, Y. You, A. Shoustikov, S. Sibley, M. E. Thompson and S. R. Forrest, Nature, 1998, 395, 151.

54 X. Yang, G. Zhou and W. Y. Wong, Chem. Soc. Rev., 2015, 44, 8484 . 
55 A. J. Huckaba and M. K. Nazeeruddin, Comments Inorg. Chem., 2016, 37, 117.

56 K. Li, G. S. M. Tong, Q. Wan, G. Cheng, W. Y. Tong, W. H. Ang, W. L. Kwong and C. M. Che, Chem. Sci., 2016, 7, 1653.

57 M. C. Tang, A. K. W. Chan, M. Y. Chan and V. W. W. Yam, Top. Curr. Chem., 2016, 374, 46.

58 J.-L. Liao, Y. Chi, C.-C. Yeh, H.-C. Kao, C.-H. Chang, M. A. Fox, P. J. Low and G.-H. Lee, J. Mater. Chem. C, 2015, 3, 4910.

59 T. Yu, D. P. K. Tsang, V. K. M. Au, W. H. Lam, M. Y. Chan and V. W. W. Yam, Chem. - Eur. J., 2013, 19, 13418.

60 S. Ranjan, S. Y. Lin, K. C. Hwang, Y. Chi, W. L. Ching, C. S. Liu, Y. T. Tao, C. H. Chien, S. M. Peng and G. H. Lee, Inorg. Chem., 2003, 42, 1248.

61 Y. L. Tung, S. W. Lee, Y. Chi, L. S. Chen, C. F. Shu, F. I. Wu, A. J. Carty, P. T. Chou, S. M. Peng and G. H. Lee, Adv. Mater., 2005, 17, 1059.

62 Z. Liu, J. Qiu, F. Wei, J. Wang, X. Liu, M. G. Helander, S. Rodney, Z. Wang, Z. Bian, Z. Lu, M. E. Thompson and C. Huang, Chem. Mater., 2014, 26, 2368.

63 F. Wu, J. Li, H. Tong, Z. Li, C. Adachi, A. Langlois, P. D. Harvey, L. Liu, W.-Y. Wong, W.-K. Wong and X. Zhu, J. Mater. Chem. C, 2015, 3, 138.

64 Q. Wei, N. Fei, A. Islam, T. Lei, L. Hong, R. Peng, X. Fan, L. Chen, P. Gao and Z. Ge, Adv. Opt. Mater., 2018, 6, 1800512 .

65 R. Hamze, J. L. Peltier, D. Sylvinson, M. Jung, J. Cardenas, R. Haiges, M. Soleilhavoup, R. Jazzar, P. I. Djurovich, G. Bertrand and M. E. Thompson, Science, 2019, 363, 601.

66 M. Osawa, M. Hoshino, M. Hashimoto, I. Kawata, S. Igawa and M. Yashima, Dalton Trans., 2015, 44, 8369.

67 Y. Liu, S.-C. Yiu, C.-L. Ho and W.-Y. Wong, Coord. Chem. Rev., 2018, 375, 514.

68 M. Wallesch, D. Volz, C. Fléchon, D. M. Zink, S. Bräse and T. Baumann, Proc. SPIE, 2014, 9183, 918309.

69 M. Hack, J. J. Brown, M. S. Weaver and M. Premutico, Lifetime OLED display, US Pat., 2016, 9257665 B2.

70 C. Adachi, R. C. Kwong, P. Djurovich, V. Adamovich, M. A. Baldo, M. E. Thompson and S. R. Forrest, Appl. Phys. Lett., 2001, 79, 2082.

71 J. Lee, C. Jeong, T. Batagoda, C. Coburn, M. E. Thompson and S. R. Forrest, Nat. Commun., 2017, 8, 15566.

72 N. C. Giebink, B. W. D’Andrade, M. S. Weaver, P. B. Mackenzie, J. J. Brown, M. E. Thompson and S. R. Forrest, J. Appl. Phys., 2008, 103, 044509.

73 N. C. Giebink, B. W. D'Andrade, M. S. Weaver, J. J. Brown and S. R. Forrest, J. Appl. Phys., 2009, 105, 124514.

74 Q. Wang and H. Aziz, ACS Appl. Mater. Interfaces, 2013, 5, 8733.

75 W. Song and J. Y. Lee, Adv. Opt. Mater., 2017, 5, 1600901.

76 R. Kabe, N. Notsuka, K. Yoshida and C. Adachi, Adv. Mater., 2016, 28, 655.

77 W. Zhao, Z. He, J. W. Y. Lam, Q. Peng, H. Ma, Z. Shuai, G. Bai, J. Hao and B. Z. Tang, Chem, 2016, 1, 592.

78 S. Reineke, N. Seidler, S. R. Yost, F. Prins, W. A. Tisdale and M. A. Baldo, Appl. Phys. Lett., 2013, 103, 093302.
79 J. Chen, T. Yu, E. Ubba, Z. Xie, Z. Yang, Y. Zhang, S. Liu, J. Xu, M. P. Aldred and Z. Chi, Adv. Opt. Mater., 2019, 7, 1801593.

80 S. Scypinski and L. C. Love, Anal. Chem., 1984, 56, 322.

81 K. Horie, K. Morishita and I. Mita, Macromolecules, 1984, 17, 1746.

82 G. Oster, N. Geacintov and A. U. Khan, Nature, 1962, 196, 1089.

83 H. A. Al-Attar and A. P. Monkman, Adv. Funct. Mater., 2012, 22, 3824 .

84 I. Salzmann, G. Heimel, M. Oehzelt, S. Winkler and N. Koch, Acc. Chem. Res., 2016, 49, 370.

85 B. Lüssem, M. Riede and K. Leo, Phys. Status Solidi A, 2013, 210, 9.

86 K. Walzer, B. Maennig, M. Pfeiffer and K. Leo, Chem. Rev., 2007, 107, 1233.

87 J. Meyer, S. Hamwi, M. Kroger, W. Kowalsky, T. Riedl and A. Kahn, Adv. Mater., 2012, 24, 5408.

88 M. Kröger, S. Hamwi, J. Meyer, T. Riedl, W. Kowalsky and A. Kahn, Org. Electron., 2009, 10, 932.

89 C. C. Chang, M. T. Hsieh, J. F. Chen, S. W. Hwang and C. H. Chen, Appl. Phys. Lett., 2006, 89, 253504.

90 S. J. Yoo, J. H. Chang, J.-H. Lee, C. K. Moon, C. I. Wu and J. J. Kim, Sci. Rep., 2014, 4, 3902.

91 Y. H. Deng, Y. Q. Li, Q. D. Ou, Q. K. Wang, F. Z. Sun, X. Y. Chen and J. X. Tang, Org. Electron., 2014, 15, 1215.

92 K. S. Yook, S. O. Jeon, S. Y. Min, J. Y. Lee, H. J. Yang, T. Noh, S. K. Kang and T. W. Lee, Adv. Funct. Mater., 2010, 20, 1797.

93 H. X. Wei, Q.-D. Ou, Z. Zhang, J. Li, Y. Q. Li, S. T. Lee and J. X. Tang, Org. Electron., 2013, 14, 839.

94 Y. Cai, H. Wei, J. Li, Q. Bao, X. Zhao, S. T. Lee, Y. Q. Li and J. X. Tang, Appl. Phys. Lett., 2011, 98, 113304.

95 J. P. Yang, Y. Xiao, Y. H. Deng, S. Duhm, N. Ueno, S. T. Lee, Y. Q. Li and J. X. Tang, Adv. Funct. Mater., 2012, 22, 600.

96 A. L. Shi, Y. Q. Li, Z. Q. Xu, F. Z. Sun, J. Li, X. B. Shi, H. X. Wei, S. T. Lee, S. Kera, N. Ueno and J. X. Tang, Org. Electron., 2013, 14, 1844.

97 Z. Q. Xu, J. P. Yang, F. Z. Sun, S. T. Lee, Y. Q. Li and J. X. Tang, Org. Electron., 2012, 13, 697.

98 G. He, O. Schneider, D. Qin, X. Zhou, M. Pfeiffer and K. Leo, J. Appl. Phys., 2004, 95, 5773.

99 H. Sasabe and J. Kido, J. Mater. Chem. C, 2013, 1, 1699.

100 L. S. Liao, W. K. Slusarek, T. K. Hatwar, M. L. Ricks and D. L. Comfort, Adv. Mater., 2008, 20, 324.

101 Q. D. Ou, C. Li, Y. Q. Li and J. X. Tang, J. Electron Spectrosc. Relat. Phenom., 2015, 204, 186.

102 J. X. Tang, M. K. Fung, C. S. Lee and S. T. Lee, J. Mater. Chem., 2010, 20, 2539.

103 L. S. Liao, K. P. Klubek and C. W. Tang, Appl. Phys. Lett., 2004, 84, 167.

104 T. Matsumoto, T. Nakada, J. Endo, K. Mori, N. Kawamura, A. Yokoi and J. Kido, SID Symp. Dig. Tech. Pap., 2003, 34, 979.

105 Y. H. Deng, Q. D. Ou, Q. K. Wang, H. X. Wei, Y. Q. Li, S. T. Lee and J. X. Tang, J. Mater. Chem. C, 2014, 2, 1982. 
106 T. Chiba, Y. J. Pu, R. Miyazaki, K.-i. Nakayama, H. Sasabe and J. Kido, Org. Electron., 2011, 12, 710.

107 L. Zhou, Q. D. Ou, J. D. Chen, S. Shen, J. X. Tang, Y. Q. Li and S. T. Lee, Sci. Rep., 2014, 4, 4040.

108 Q. D. Ou, L. Zhou, Y. Q. Li, J. D. Chen, C. Li, S. Shen and J. X. Tang, Adv. Opt. Mater., 2015, 3, 87.

109 K. Goushi and C. Adachi, Appl. Phys. Lett., 2012, 101, 023306.

110 Z. Chen, X. K. Liu, C. J. Zheng, J. Ye, C. L. Liu, F. Li, X. M. Ou, C. S. Lee and X. H. Zhang, Chem. Mater., 2015, 27, 5206.

111 X. K. Liu, Z. Chen, C. J. Zheng, C. L. Liu, C. S. Lee, F. Li, X. M. Ou and X. H. Zhang, Adv. Mater., 2015, 27, 2378.

112 W. Y. Hung, G. C. Fang, Y. C. Chang, T. Y. Kuo, P. T. Chou, S. W. Lin and K. T. Wong, ACS Appl. Mater. Interfaces, 2013, $5,6826$.

113 D. Graves, V. Jankus, F. B. Dias and A. Monkman, Adv. Funct. Mater., 2014, 24, 2343.

114 X. K. Liu, Z. Chen, C. J. Zheng, M. Chen, W. Liu, X. H. Zhang and C. S. Lee, Adv. Mater., 2015, 27, 2025.

115 J. W. Sun, J. H. Lee, C. K. Moon, K. H. Kim, H. Shin and J. J. Kim, Adv. Mater., 2014, 26, 5684.

116 X. Lin, Y. Zhu, B. Zhang, X. Zhao, B. Yao, Y. Cheng, Z. Li, Y. Qu and Z. Xie, ACS Appl. Mater. Interfaces, 2018, 10, 47.

117 S.-F. Wu, S.-H. Li, Y.-K. Wang, C.-C. Huang, Q. Sun, J.-J. Liang, L.-S. Liao and M.-K. Fung, Adv. Funct. Mater., 2017, 27, 1701314.

118 J. Zhao, S. Yuan, X. Du, W. Li, C. Zheng, S. Tao and X. Zhang, Adv. Opt. Mater., 2018, 6, 1800825.

119 Y.-S. Park, S. Lee, K.-H. Kim, S.-Y. Kim, J.-H. Lee and J.-J. Kim, Adv. Funct. Mater., 2013, 23, 4914.

120 Y. Seino, H. Sasabe, Y. J. Pu and J. Kido, Adv. Mater., 2014, 26, 1612.

121 H. Nakanotani, T. Higuchi, T. Furukawa, K. Masui, K. Morimoto, M. Numata, H. Tanaka, Y. Sagara, T. Yasuda and C. Adachi, Nat. Commun., 2014, 5, 4016.

122 D. Zhang, L. Duan, C. Li, Y. Li, H. Li, D. Zhang and Y. Qiu, Adv. Mater., 2014, 26, 5050.

123 W. Song, I. Lee and J. Y. Lee, Adv. Mater., 2015, 27, 4358.

124 T. Furukawa, H. Nakanotani, M. Inoue and C. Adachi, Sci. Rep., 2015, 5, 8429.

125 D. Zhang, X. Song, M. Cai and L. Duan, Adv. Mater., 2018, 30, 1705250.

126 P. Heimel, A. Mondal, F. May, W. Kowalsky, C. Lennartz, D. Andrienko and R. Lovrincic, Nat. Commun., 2018, 9, 4990.

127 S. Y. Byeon, D. R. Lee, K. S. Yook and J. Y. Lee, Adv. Mater., 2019, 31, e1803714.

128 G. Gu, D. Z. Garbuzov, P. E. Burrows, S. Venkatesh, S. R. Forrest and M. E. Thompson, Opt. Lett., 1997, 22, 396.

129 N. C. Greenham, R. H. Friend and D. D. C. Bradley, Adv. Mater., 1994, 6, 491.

130 V. Bulović, V. B. Khalfin, G. Gu, P. E. Burrows, D. Z. Garbuzov and S. R. Forrest, Phys. Rev. B: Condens. Matter Mater. Phys., 1998, 58, 3730.

131 Y.-J. Lee, S.-H. Kim, J. Huh, G.-H. Kim, Y.-H. Lee, S.-H. Cho, Y.-C. Kim and Y. R. Do, Appl. Phys. Lett., 2003, 82, 3779.
132 A. Chutinan, K. Ishihara, T. Asano, M. Fujita and S. Noda, Org. Electron., 2005, 6, 3.

$133 \mathrm{~J}$. Hauss, T. Bocksrocker, B. Riedel, U. Lemmer and M. Gerken, Opt. Express, 2011, 19, A851.

134 L. H. Smith, J. A. E. Wasey, I. D. W. Samuel and W. L. Barnes, Adv. Funct. Mater., 2005, 15, 1839.

135 S. Nowy, B. C. Krummacher, J. Frischeisen, N. A. Reinke and W. Brütting, J. Appl. Phys., 2008, 104, 123109.

136 S. Möller and S. R. Forrest, J. Appl. Phys., 2002, 91, 3324.

137 M. G. Helander, Z. B. Wang, J. Qiu, M. T. Greiner, D. P. Puzzo, Z. W. Liu and Z. H. Lu, Science, 2011, 332, 944.

138 J. P. Yang, Q. Y. Bao, Z. Q. Xu, Y. Q. Li, J. X. Tang and S. Shen, Appl. Phys. Lett., 2010, 97, 223303.

139 M. Thomschke, S. Reineke, B. Lussem and K. Leo, Nano Lett., 2012, 12, 424.

140 B. Liu, L. Wang, M. Xu, H. Tao, D. Gao, J. Zou, L. Lan, H. Ning, J. Peng and Y. Cao, J. Mater. Chem. C, 2014, 2, 9836.

141 W. Zhu, X. Wu, W. Sun, L. Sun, K. Guo, M. Tang and P. Zhou, Org. Electron., 2014, 15, 1113.

142 J. M. Lupton, B. J. Matterson, I. D. W. Samuel, M. J. Jory and W. L. Barnes, Appl. Phys. Lett., 2000, 77, 3340.

143 B. J. Matterson, J. M. Lupton, A. F. Safonov, M. G. Salt, W. L. Barnes and I. D. W. Samuel, Adv. Mater., 2001, 13, 123.

144 J. M. Ziebarth and M. D. McGehee, J. Appl. Phys., 2005, 97, 064502 .

145 J. M. Ziebarth, A. K. Saafir, S. Fan and M. D. McGehee, Adv. Funct. Mater., 2004, 14, 451.

146 S. M. Jeong, F. Araoka, Y. Machida, K. Ishikawa, H. Takezoe, S. Nishimura and G. Suzaki, Appl. Phys. Lett., 2008, 92, 083307.

147 L. Zhou, X. Jiang, Y. Li, A. Shi, J. Chen, Q. Ou, H. Liu and J. Tang, ACS Appl. Mater. Interfaces, 2014, 6, 18139.

148 X.-L. Zhang, J. Feng, J.-F. Song, X.-B. Li and H.-B. Sun, Opt. Lett., 2011, 36, 3915.

149 Y. Bai, J. Feng, Y.-F. Liu, J.-F. Song, J. Simonen, Y. Jin, Q.-D. Chen, J. Zi and H.-B. Sun, Org. Electron., 2011, 12, 1927.

150 Y. G. Bi, J. Feng, Y. F. Li, X. L. Zhang, Y. F. Liu, Y. Jin and H. B. Sun, Adv. Mater., 2013, 25, 6969.

151 C. Y. Chen, W. K. Lee, Y. J. Chen, C. Y. Lu, H. Y. Lin and C. C. Wu, Adv. Mater., 2015, 27, 4883.

152 W. H. Koo, S. M. Jeong, F. Araoka, K. Ishikawa, S. Nishimura, T. Toyooka and H. Takezoe, Nat. Photonics, 2010, 4, 222.

153 P. F. Little, B. E. Avis and R. B. Turner, Plasma Phys., 1970, $12,565$.

154 M. Fujita, T. Ueno, K. Ishihara, T. Asano, S. Noda, H. Ohata, T. Tsuji, H. Nakada and N. Shimoji, Appl. Phys. Lett., 2004, 85, 5769.

155 Y. H. Kim, J. Lee, W. M. Kim, C. Fuchs, S. Hofmann, H.-W. Chang, M. C. Gather, L. Müller-Meskamp and K. Leo, Adv. Funct. Mater., 2014, 24, 2553.

156 Q. D. Ou, L. Zhou, Y. Q. Li, S. Shen, J.-D. Chen, C. Li, Q. K. Wang, S. T. Lee and J. X. Tang, Adv. Funct. Mater., 2014, 24, 7249.

157 L. Zhou, Q. D. Ou, Y. Q. Li, H. Y. Xiang, L. H. Xu, J. D. Chen, C. Li, S. Shen, S. T. Lee and J. X. Tang, Adv. Funct. Mater., 2015, 25, 2660. 
158 Y. Li, M. Kovacic, J. Westphalen, S. Oswald, Z. Ma, C. Hanisch, P. A. Will, L. Jiang, M. Junghaehnel, R. Scholz, S. Lenk and S. Reineke, Nat. Commun., 2019, 10, 2972.

159 K. Y. Yang, K. C. Choi and C. W. Ahn, Appl. Phys. Lett., 2009, 94, 173301.

160 Y. Gu, D. D. Zhang, Q. D. Ou, Y. H. Deng, J. J. Zhu, L. Cheng, Z. Liu, S. T. Lee, Y. Q. Li and J. X. Tang, J. Mater. Chem. C, 2013, 1, 4319.

161 R. Grover, R. Srivastava, M. N. Kamalasanan and D. S. Mehta, Phys. Status Solidi RRL, 2014, 8, 81.

162 C. Lee, D. J. Kang, H. Kang, T. Kim, J. Park, J. Lee, S. Yoo and B. J. Kim, Adv. Energy Mater., 2014, 4, 1301345.

163 D. H. Kim and T. W. Kim, Opt. Express, 2015, 23, 11211.

164 D. H. Kim and T. W. Kim, Org. Electron., 2016, 34, 262.

165 K. Tong, X. Liu, F. Zhao, D. Chen and Q. Pei, Adv. Opt. Mater., 2017, 5, 1700307.

166 Y. Sun and S. R. Forrest, Nat. Photonics, 2008, 2, 483.

167 J. Kim, Y. Qu, C. Coburn and S. R. Forrest, ACS Photonics, 2018, 5, 3315.

168 E. Kim, H. Cho, K. Kim, T. W. Koh, J. Chung, J. Lee, Y. Park and S. Yoo, Adv. Mater., 2015, 27, 1624.

169 A. Salehi, Y. Chen, X. Fu, C. Peng and F. So, ACS Appl. Mater. Interfaces, 2018, 10, 9595.

170 A. Ingenito, O. Isabella and M. Zeman, Prog. Photovoltaics, 2015, 23, 1649.

171 K. Liu and L. Jiang, Nano Today, 2011, 6, 155.

172 A. Bay, N. André, M. Sarrazin, A. Belarouci, V. Aimez, L. A. Francis and J. P. Vigneron, Opt. Express, 2013, 21, A179.

173 R. J. Martín-Palma, A. E. Miller, D. P. Pulsifer and A. Lakhtakia, Appl. Phys. Lett., 2014, 105, 103703.

174 L. Zhou, X. Dong, Y. Zhou, W. Su, X. Chen, Y. Zhu and S. Shen, ACS Appl. Mater. Interfaces, 2015, 7, 26989.

175 J. J. Kim, J. Lee, S. P. Yang, H. G. Kim, H. S. Kweon, S. Yoo and K. H. Jeong, Nano Lett., 2016, 16, 2994.

176 Y.-W. Lim, O. E. Kwon, S.-M. Kang, H. Cho, J. Lee, Y.-S. Park, N. S. Cho, W.-Y. Jin, J. Lee, H. Lee, J.-W. Kang, S. Yoo, J. Moon and B.-S. Bae, Adv. Funct. Mater., 2018, 28, 1802944.

177 D. Yokoyama, A. Sakaguchi, M. Suzuki and C. Adachi, Org. Electron., 2009, 10, 127.

178 D. Yokoyama, J. Mater. Chem., 2011, 21, 19187.

179 J. Frischeisen, D. Yokoyama, A. Endo, C. Adachi and W. Brütting, Org. Electron., 2011, 12, 809.

180 P. Liehm, C. Murawski, M. Furno, B. Lüssem, K. Leo and M. C. Gather, Appl. Phys. Lett., 2012, 101, 253304.

181 C.-Y. Lu, M. Jiao, W.-K. Lee, C.-Y. Chen, W.-L. Tsai, C.-Y. Lin and C.-C. Wu, Adv. Funct. Mater., 2016, 26, 3250.

182 K.-H. Kim, E. S. Ahn, J.-S. Huh, Y.-H. Kim and J.-J. Kim, Chem. Mater., 2016, 28, 7505.

183 S. Y. Byeon, J. Kim, D. R. Lee, S. H. Han, S. R. Forrest and J. Y. Lee, Adv. Opt. Mater., 2018, 6, 1701340.

184 S. Kwon, H. Kim, S. Choi, E. G. Jeong, D. Kim, S. Lee, H. S. Lee, Y. C. Seo and K. C. Choi, Nano Lett., 2018, 18, 347.

185 T. Kim, J. S. Price, A. Grede, S. Lee, G. Choi, W. Guan, T. N. Jackson and N. C. Giebink, Adv. Mater. Technol., 2018, 3, 1800067.
186 K. Ellmer, Nat. Photonics, 2012, 6, 809.

187 A. Kumar and C. Zhou, ACS Nano, 2010, 4, 11.

188 S. De, T. M. Higgins, P. E. Lyons, E. M. Doherty, P. N. Nirmalraj, W. J. Blau, J. J. Boland and J. N. Coleman, ACS Nano, 2009, 3, 1767.

189 E. C. Garnett, W. Cai, J. J. Cha, F. Mahmood, S. T. Connor, M. Greyson Christoforo, Y. Cui, M. D. McGehee and M. L. Brongersma, Nat. Mater., 2012, 11, 241.

190 J. H. Yim, S.-y. Joe, C. Pang, K. M. Lee, H. Jeong, J.-Y. Park, Y. H. Ahn, J. C. de Mello and S. Lee, ACS Nano, 2014, 8, 2857.

191 J.-Y. Lee, S. T. Connor, Y. Cui and P. Peumans, Nano Lett., 2008, 8, 689.

192 C.-H. Liu and X. Yu, Nanoscale Res. Lett., 2011, 6, 75.

193 R. Zhu, C.-H. Chung, K. C. Cha, W. Yang, Y. B. Zheng, H. Zhou, T.-B. Song, C.-C. Chen, P. S. Weiss, G. Li and Y. Yang, ACS Nano, 2011, 5, 9877.

194 V. Scardaci, R. Coull, P. E. Lyons, D. Rickard and J. N. Coleman, Small, 2011, 7, 2621.

195 J. Choi, Y. S. Shim, C. H. Park, H. Hwang, J. H. Kwack, D. J. Lee, Y. W. Park and B. K. Ju, Small, 2018, 14, 1702567.

196 W. Gaynor, S. Hofmann, M. G. Christoforo, C. Sachse, S. Mehra, A. Salleo, M. D. McGehee, M. C. Gather, B. Lüssem, L. Müller-Meskamp, P. Peumans and K. Leo, Adv. Mater., 2013, 25, 4006.

197 Y. Huang, Y. Liu, K. Youssef, K. Tong, Y. Tian and Q. Pei, Adv. Opt. Mater., 2018, 6, 1801015.

198 W. Li, Y. Q. Li, Y. Shen, Y. X. Zhang, T. Y. Jin, J. D. Chen, X. H. Zhang and J. X. Tang, Adv. Opt. Mater., 2019, 7, 1900985.

199 C. Zhang, A. Khan, J. Cai, C. Liang, Y. Liu, J. Deng, S. Huang, G. Li and W. D. Li, ACS Appl. Mater. Interfaces, 2018, 10, 21009.

200 S. Han, Y. Chae, J. Y. Kim, Y. Jo, S. S. Lee, S.-H. Kim, K. Woo, S. Jeong, Y. Choi and S. Y. Lee, J. Mater. Chem. C, 2018, 6, 4389.

201 L. Zhou, H. Y. Xiang, S. Shen, Y. Q. Li, J. D. Chen, H. J. Xie, I. A. Goldthorpe, L. S. Chen, S. T. Lee and J. X. Tang, ACS Nano, 2014, 8, 12796.

202 H. Y. Xiang, Y. Q. Li, L. Zhou, H. J. Xie, C. Li, Q. D. Ou, L. S. Chen, C. S. Lee, S. T. Lee and J. X. Tang, ACS Nano, 2015, 9, 7553 .

203 T. Schwab, S. Schubert, L. Müller-Meskamp, K. Leo and M. C. Gather, Adv. Opt. Mater., 2013, 1, 921.

204 Z. B. Wang, M. G. Helander, J. Qiu, D. P. Puzzo, M. T. Greiner, Z. M. Hudson, S. Wang, Z. W. Liu and Z. H. Lu, Nat. Photonics, 2011, 5, 753.

205 Q. D. Ou, L. H. Xu, W. Y. Zhang, Y. Q. Li, Y. B. Zhang, X. D. Zhao, J. D. Chen and J. X. Tang, Opt. Express, 2016, 24, A674.

206 L. H. Xu, Q. D. Ou, Y. Q. Li, Y. B. Zhang, X. D. Zhao, H. Y. Xiang, J. D. Chen, L. Zhou, S. T. Lee and J. X. Tang, ACS Nano, 2016, 10, 1625.

207 T.-H. Han, Y. Lee, M.-R. Choi, S.-H. Woo, S.-H. Bae, B. H. Hong, J.-H. Ahn and T.-W. Lee, Nat. Photonics, 2012, 6, 105. 
208 N. Li, S. Oida, G. S. Tulevski, S. J. Han, J. B. Hannon, D. K. Sadana and T. C. Chen, Nat. Commun., 2013, 4, 2294.

209 J. Lee, T. H. Han, M. H. Park, D. Y. Jung, J. Seo, H. K. Seo, H. Cho, E. Kim, J. Chung, S. Y. Choi, T. S. Kim, T. W. Lee and S. Yoo, Nat. Commun., 2016, 7, 11791.

210 J. Liang, L. Li, X. Niu, Z. Yu and Q. Pei, Nat. Photonics, 2013, $7,817$.

211 Z. Yu, X. Niu, Z. Liu and Q. Pei, Adv. Mater., 2011, 23, 3989.

212 T. Sekitani, H. Nakajima, H. Maeda, T. Fukushima, T. Aida, K. Hata and T. Someya, Nat. Mater., 2009, 8, 494.

213 J. Liang, L. Li, D. Chen, T. Hajagos, Z. Ren, S.-Y. Chou, W. Hu and Q. Pei, Nat. Commun., 2015, 6, 7647.

214 D. Yin, J. Feng, R. Ma, Y. F. Liu, Y. L. Zhang, X. L. Zhang, Y. G. Bi, Q. D. Chen and H. B. Sun, Nat. Commun., 2016, 7, 11573.

215 S. Jung, S. Lee, M. Song, D.-G. Kim, D. S. You, J.-K. Kim, C. S. Kim, T.-M. Kim, K.-H. Kim, J.-J. Kim and J.-W. Kang, Adv. Energy Mater., 2014, 4, 1300474.

216 H. Zheng, Y. Zheng, N. Liu, N. Ai, Q. Wang, S. Wu, W. Xu, F. Hung, J. B. Peng and Y. Cao, Nat. Commun., 2013, 4, 1971.

217 D. Braun and A. J. Heeger, Appl. Phys. Lett., 1991, 58, 1982.

218 T. R. Hebner, C. C. Wu, D. Marcy, M. H. Lu and J. C. Sturm, Appl. Phys. Lett., 1998, 72, 519.

219 C. D. Müller, A. Falcou, N. Reckefuss, M. Rojahn, V. Wiederhirn, P. Rudati and K. Meerholz, Nature, 2003, 421, 829.

220 A. Sandström, H. F. Dam, F. C. Krebs and L. Edman, Nat. Commun., 2012, 3, 1002.

221 M. Cai, T. Xiao, E. Hellerich, Y. Chen, R. Shinar and J. Shinar, Adv. Mater., 2011, 23, 3590.

222 X. Zhao, S. Wang, J. You, Y. Zhang and X. Li, J. Mater. Chem. C, 2015, 3, 11377.

223 T. Chiba, Y. J. Pu and J. Kido, Adv. Mater., 2015, 27, 4681.

224 A. E. Nikolaenko, M. Cass, F. Bourcet, D. Mohamad and M. Roberts, Adv. Mater., 2015, 27, 7236.

225 X. L. Chen, J. H. Jia, R. Yu, J. Z. Liao, M. X. Yang and C. Z. Lu, Angew. Chem., Int. Ed., 2017, 56, 15006.

226 Y. Wada, S. Kubo and H. Kaji, Adv. Mater., 2018, 30, 1705641.

227 Y. J. Cho, B. D. Chin, S. K. Jeon and J. Y. Lee, Adv. Funct. Mater., 2015, 25, 6786.

228 K. Albrecht, K. Matsuoka, K. Fujita and K. Yamamoto, Angew. Chem., Int. Ed., 2015, 54, 5677.

229 A. Verma, D. M. Zink, C. Fléchon, J. L. Carballo, H. Flügge, J. M. Navarro and D. Volz, Appl. Phys. A: Mater. Sci. Process., 2016, 122, 191.

230 S. Y. Lee, T. Yasuda, H. Komiyama, J. Lee and C. Adachi, Adv. Mater., 2016, 28, 4019.

231 J. Kido, K. Hongawa, K. Okuyama and K. Nagai, Appl. Phys. Lett., 1994, 64, 815.

232 Y. Yin, M. U. Ali, W. Xie, H. Yang and H. Meng, Mater. Chem. Front., 2019, 3, 970.

233 T. L. Dawson, Color. Technol., 2010, 126, 1.

234 S. Beaupré, P. L. T. Boudreault and M. Leclerc, Adv. Mater., 2010, 22, E6.
235 S. Reineke, Nat. Mater., 2015, 14, 459.

236 V. Adamovich, J. Brooks, A. Tamayo, A. M. Alexander, P. I. Djurovich, B. W. D'Andrade, C. Adachi, S. R. Forrest and M. E. Thompson, New J. Chem., 2002, 26, 1171.

237 B. W. D'Andrade, R. J. Holmes and S. R. Forrest, Adv. Mater., 2004, 16, 624.

238 G. Schwartz, M. Pfeiffer, S. Reineke, K. Walzer and K. Leo, Adv. Mater., 2007, 19, 3672.

239 S. Reineke, F. Lindner, G. Schwartz, N. Seidler, K. Walzer, B. Lussem and K. Leo, Nature, 2009, 459, 234.

240 K. Yamae, H. Tsuji, V. Kittichungchit, N. Ide and T. Komoda, SID Symp. Dig. Tech. Pap., 2013, 44, 916.

241 S. Jeon, S. Lee, K.-H. Han, H. Shin, K.-H. Kim, J.-H. Jeong and J.-J. Kim, Adv. Opt. Mater., 2018, 6, 1701349.

242 H. Y. Xiang, Y. Q. Li, S. S. Meng, C. S. Lee, L. S. Chen and J. X. Tang, Adv. Opt. Mater., 2018, 6, 1800831.

243 A. R. Duggal, J. J. Shiang, D. F. Foust, L. G. Turner, W. F. Nealon and J. C. Bortscheller, SID Symp. Dig. Tech. Pap., 2005, 36, 28.

244 F. So, J. Kido and P. Burrows, MRS Bull., 2008, 33, 663.

245 T. Komoda, H. Tsuji, N. Ito, T. Nishimori and N. Ide, SID Symp. Dig. Tech. Pap., 2010, 41, 993.

246 P. A. Levermore, V. Adamovich, K. Rajan, W. Yeager, C. Lin, S. Xia, G. S. Kottas, M. S. Weaver, R. Kwong, R. Ma, M. Hack and J. J. Brown, SID Symp. Dig. Tech. Pap., 2010, 41, 786.

247 J. Spindler, M. Kondakova, M. Boroson, M. Büchel, J. Eser and J. Knipping, SID Symp. Dig. Tech. Pap., 2018, 49, 1135.

248 T. Kim, J. S. Price, A. Grede, S. Lee, G. Choi, W. Guan, T. N. Jackson and N. C. Giebink, Adv. Mater. Technol., 2018, 3, 1800067.

249 T. Tsujimura, OLED Display Fundamentals and Applications, 2nd edn, 2017, vol. 9, p. 255, DOI: 10.1002/9781119187493.ch9.

250 Philips interactive OLED mirror. http:/www.osadirect. com/news/article/821/philips-launches-livingshapes-aninteractive-oled-mirror/.

251 Alkilu portable OLED light panel. http://www.alkilu.com/.

252 Japan-based Stanley Electric developed an OLED tail light prototype. http://www.talkoled.com/tag/automotive/page/2/. 253 K. Thapan, J. Arendt and D. J. Skene, J. Physiol., 2001, 535, 261.

254 M. Sato, T. Sakaguchi and T. Morita, Biol. Rhythm Res., 2005, 36, 287.

255 J. H. Jou, M. H. Wu, S. M. Shen, H. C. Wang, S. Z. Chen, S. H. Chen and Y. L. Hsieh, Appl. Phys. Lett., 2009, 95, 013307.

256 J. Yu, H. Lin, F. Wang, Y. Lin, J. Zhang, H. Zhang, Z. Wang and B. Wei, J. Mater. Chem., 2012, 22, 22097.

257 J. H. Wu, C. A. Chi, C. L. Chiang, G. Y. Chen, Y. P. Lin, C. C. Chen and J. Y. Li, Opt. Mater., 2016, 55, 90.

258 J. H. Jou, S. H. Chen, S. M. Shen, Y. C. Jou, C. H. Lin, S. H. Peng and C. C. Wang, J. Mater. Chem., 2011, 21, 17850.

259 J. H. Jou, C. Y. Hsieh, J. R. Tseng, S. H. Peng, Y. C. Jou, J. H. Hong and C. H. Lin, Adv. Funct. Mater., 2013, 23, 2750.

260 C. Adachi, M. A. Baldo, S. R. Forrest and M. E. Thompson, Appl. Phys. Lett., 2000, 77, 904.

261 Q. Huang, R. Meerheim, K. Fehse, G. Schwartz, S. Reineke, K. Walzer and K. Leo, SID Symp. Dig. Tech. Pap., 2007, 38, 1282. 
262 H. Jia, Natl. Sci. Rev., 2018, 5, 427.

263 Y. K. Jung, H. S. Choi, S. Y. Ahn, S. Kim, H. Choi, C. W. Han, B. Kim, S. J. Kim, J. Kim, J. H. Choi, S. Yoon, Y. H. Tak, H. C. Choi, B. C. Ahn and I. B. Kang, SID Symp. Dig. Tech. Pap., 2016, 47, 707.

264 R. Tani, J.-S. Yoon, S.-I. Yun, W.-J. Nam, S. Takasugi, J.-M. Kim, J.-K. Park, S.-Y. Kwon, P.-Y. Kim, C.-H. Oh and B.-C. Ahn, SID Symp. Dig. Tech. Pap., 2015, 46, 950.

265 Sony Audio player. http://www.sony.jp/walkman/products/ NW-A850_series/.

266 Sony A99II digital camera. http://thenewcamera.com/tag/ sony-a99-ii/.

267 Apple watch series 5. https://www.apple.com/cn/apple-watchseries-5/.

268 Apple iPhone X, https://www.oled-info.com/apple-announcesiphone-x-58-flexible-super-amoled, 2017.

269 C. W. Han, K. M. Kim, S. J. Bae, H. S. Choi, J. M. Lee, T. S. Kim, Y. H. Tak, S. Y. Cha and B. C. Ahn, SID Symp. Dig. Tech. Pap., 2012, 43, 279.
270 C. Y. Chen, L. F. Lin, J. Y. Lee, W. H. Wu, S. C. Wang, Y. M. Chiang, Y. H. Chen, C. C. Chen, Y. H. Chen, C. L. Chen, T. H. Shih, C. H. Liu, H. C. Ting, H. H. Lu, L. Tsai, H. S. Lin, L. H. Chang and Y. H. Lin, SID Symp. Dig. Tech. Pap., 2013, 44, 247.

271 H. J. Shin, S. Takasugi, K. M. Park, S. H. Choi, Y. S. Jeong, B. C. Song, H. S. Kim, C. H. Oh and B. C. Ahn, SID Symp. Dig. Tech. Pap., 2015, 46, 53.

272 C. W. Han, M. Y. Han, S. R. Joung, J. S. Park, Y. K. Jung, J. M. Lee, H. S. Choi, G. J. Cho, D. H. Kim, M. K. Yee, H. G. Kim, H. C. Choi, C. H. Oh and I. B. Kang, SID Symp. Dig. Tech. Pap., 2017, 48, 1.

273 H. J. Shin, S. Takasugi, K. M. Park, S. H. Choi, Y. S. Jeong, H. S. Kim, C. H. Oh and B. C. Ahn, SID Symp. Dig. Tech. Pap., 2014, 45, 720.

274 Alienware 13 laptop. https://www.cnet.com/products/ alienware-13-2016/.

275 Sony DEV 50 binocular camcorder. http://hdvcam.net/ sony/sony-dev-50-review/. 